\title{
Métodos estocásticos de otimização global para empacotar círculos em elipses
}

Luis Henrique Bustamante de Morais

\author{
DISSERTAÇÃO APRESENTADA \\ $\mathrm{AO}$ \\ Instituto DE MATEMÁticA E EstatísticA \\ DA \\ Universidade DE SÃo Paulo \\ PARA \\ OBTENÇÃO DO TÍTULO \\ $\mathrm{DE}$ \\ Mestre em CiÊnCIAS
}

Programa: Pós-Graduação em Ciência da Computação

Orientador: Prof. Dr. Ernesto G. Birgin

Durante o desenvolvimento deste trabalho o autor recebeu auxílio financeiro da $\mathrm{CNPq}$

São Paulo, 15 de maio de 2012 


\section{Métodos estocásticos de otimização global para empacotar círculos em elipses}

Esta dissertação contém as correções e alterações sugeridas pela Comissão Julgadora durante a defesa realizada por Luis Henrique Bustamante de Morais em

09/05/2012. O original encontra-se disponível no Instituto de Matemática e Estatística da Universidade de São Paulo.

Comissão Julgadora:

- Prof. Dr. Ernesto G. Birgin (orientador) - IME-USP

- Prof. Dr. Marcelo Gomes Queiroz - IME-USP

- Profa. Dra. Celma de Oliveira Ribeiro - EP-USP 
We can only see a short distance ahead, but we can see plenty there that needs to be done.

("Computing Machinery and Intelligence" de Alan Turing, 1950) 


\section{Agradecimentos}

Ao Ernesto, pela valorosa orientação fornecida durante todo o mestrado.

Aos Professores Marcelo Queiroz e Celma Ribeiro, pelas considerações feitas no presente texto.

Ao meu pai, minha mãe, meu irmão e minha irmã, pela motivação diária dada pela existência de vocês e pela grande ajuda financeira durante vários momentos, sem isso esses anos fora de casa não seriam possíveis.

Aos meus colegas cearenses do IME, Roberto, Lima, Mota e Rafael, pelo convívio durante este período e pela recepção no início do mestrado.

Aos meus colegas de apartamento, Puglia, Paulo Victor, Fredy, Fran, Vinícius e Igor, pelo convívio saudável e fraterno.

Aos meus colegas de laboratório, Ricardo, Paulo, Vivi, Esdras, Fábio, Márcio, Marcelo, Jessé, Sekeff, Street, Fabiano, Wesley, Tales, Sirley, Ellen, Lobato, Carlos, John, Erika, Boris, Mijail, Juan e tanta outras pessoas legais que eu encontrei aqui pelo IME, que foram essenciais em vários momentos e me aguentaram durante outros tantos. 


\title{
Resumo
}

\author{
Métodos estocásticos de otimização \\ global para empacotar círculos em elipses
}

Neste trabalho, consideramos uma nova parametrização para o problema de empacotar a maior quantidade possível de círculos idênticos numa região elíptica dada. Apresentamos algoritmos com propriedades de convergência global e algumas estratégias heurísticas. Ilustramos com experimentos numéricos extensivos cada uma das estratégias utilizadas.

Palavras-chave: Empacotamento, elipses, círculos, otimização global, Lagrangianos aumentados. 


\section{Abstract}

\section{Stochastic global optimization strategies for packing circles within ellipses}

In this work we consider a new parametrization for the problem of packing the maximum number of identical circles within a given elliptical region. We present algorithms with global convergence properties and some heuristic strategies. We illustrate each described strategy with extensive numerical experiments.

Keywords: Packing, ellipses, circles, global optimization, augmented Lagrangians. 


\section{Sumário}

1 Introdução 1

2 Empacotamento de círculos em círculos, poliedros e elipses $\quad 3$

2.1 Empacotamento de círculos em círculos e poliedros . . . . . . . . . . . . . . 3

2.2 Empacotamento de círculos em elipses . . . . . . . . . . . . . . . . . 3

2.2.1 Círculos dados em uma elipse fixa . . . . . . . . . . . . . . 5

2.2.2 Número máximo de círculos idênticos em uma elipse fixa . . . . . . . . . . . 5

2.2.3 Elipse de área mínima que contém um conjunto de círculos dados . . . . . . . 6

2.2.4 Bolas em um contêiner cilíndrico . . . . . . . . . . . . . . . . 6

3 Procedimentos de otimização $\quad 9$

3.1 Cálculo esparso da sobreposição . . . . . . . . . . . . . . . . . . . . . . . . 10

4 Experimentos computacionais $\quad 13$

4.1 Calculando o limite inferior $m_{l b} \ldots \ldots \ldots \ldots \ldots \ldots \ldots \ldots$

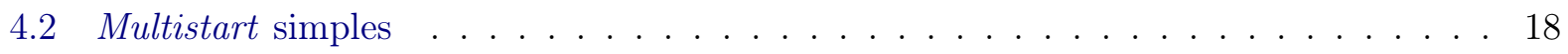

4.3 Tunneling . . . . . . . . . . . . . . . . . . . 25

4.4 Alternativas ao multistart . . . . . . . . . . . . . . . . . . . 31

4.5 Resumindo os melhores resultados . . . . . . . . . . . . . . . . . . 41

5 Conclusões $\quad 45$

$\begin{array}{ll}\text { Referências Bibliográficas } & 47\end{array}$ 


\section{Capítulo 1}

\section{Introdução}

O problema de empacotamento de itens dentro de regiões limitadas no espaço Euclidiano tem múltiplas aplicações em Física, Química, Engenharia, Geofísica e Artes. Problemas desse tipo exibem vários níveis de complexidade. Os mais fáceis envolvem o empacotamento de itens simples em domínios simples, e, algumas vezes, soluções analíticas para esses problemas existem [CS88]. Frequentemente, problemas simples motivam o interesse em aplicações recreativas [Fri11]. No outro extremo, encontramos os problemas de empacotamento de itens irregulares em domínios irregulares. Neste caso, não existem soluções fechadas, mas existem importantes aplicações em ciências ambientais. O empacotamento irregular de partículas do solo, por exemplo, determina a maneira na qual a formação de raízes das plantas ocorrem, como também o movimento da água no solo [Hog10]. Uma abordagem útil para lidar com empacotamentos irregulares é baseado no conceito de "sentinelas" [BMMR06].

A dinâmica molecular (DM) motivou o desenvolvimento de uma abordagem bem sucedida para empacotamento de moléculas em diferentes domínios do espaço Euclidiano no $\mathbb{R}^{3}$. O pacote Packmol [MABM09, MM03] emprega procedimentos de otimização [ABMS07, BM02] e heurísticas para a distribuição de moléculas em um dado domínio com a finalidade de construir simulações. Formulações de programação não linear foram aplicadas com sucesso a um vasto campo de problemas de empacotamento. Veja [BG10, BL10, BMMR06, BMNR06, BMR05, BS08] e as referências contidas nos mesmos. O grande número de aplicações motivou uma análise detalhada e experimentos com problemas geométricos de complexidade crescente. A tecnologia desenvolvida em conexão com os problemas geométricos pode posteriormente ser empregada em aplicações relevantes de larga escala, como as consideradas na área de DM.

Neste trabalho, estudamos o problema de empacotamento de círculos em elipses. No Capítulo 2, descrevemos modelos matemáticos para o problema. No Capítulo 3, os procedimentos de otimização serão introduzidos. No Capítulo 4, reportamos experimentos sobre o problema de empacotamento do número máximo de círculos que podem ser empacotados em uma elipse dada. O Capítulo 5 apresenta as conclusões deste trabalho. 


\section{Capítulo 2}

\section{Empacotamento de círculos em círculos, poliedros e elipses}

\subsection{Empacotamento de círculos em círculos e poliedros}

Muitos trabalhos recentes lidam com o problema de empacotamento do número máximo de retângulos [BL10, BMMR06, BMNR06, MB10] ou círculos idênticos [BG10, BMR05, BS08] no interior de uma variedade de regiões convexas usando técnicas de programação não linear. No caso de itens retangulares, a convexidade do contêiner permite que o encaixe do item no interior da região seja modelado de maneira trivial - é suficiente pedir que os quatro vértices do item estejam dentro da região. Quando os itens são circulares, as regiões convexas são dadas por círculos, poliedros e combinações de ambos. Nesses casos, modelar o encaixe de itens circulares no interior do contêiner também é uma tarefa simples - reduz-se a perguntar se os centros dos itens estão em uma região menor com a mesma estrutura da original (veja a Figura 2.1). Essa maneira de modelar a pertinência dos itens no contêiner não é válida para outros tipos de regiões, como elipses (veja a Figura 2.2). Modelar a pertinência dos itens circulares em uma região elíptica é uma tarefa difícil que não pode ser conseguida pelas técnicas mencionadas anteriormente.

\subsection{Empacotamento de círculos em elipses}

Nesta seção, abordamos o problema de empacotamento de discos em elipses. Consideramos a elipse definida por $x^{2} / a^{2}+y^{2} / b^{2}=1$, onde $a \geq b$. Como sabemos, esta elipse pode ser parametrizada por

$$
x=a \cos (t), y=b \sin (t), 0 \leq t \leq 2 \pi .
$$

A região sólida delimitada por essa elipse pode ser parametrizada da seguinte maneira:

$$
x=\left(a-s b^{2} / a\right) \cos (t), y=(1-s) b \sin (t), 0 \leq t \leq 2 \pi, 0 \leq s \leq 1 .
$$

Se $(x, y)$ é dado por (2.1), o ponto na fronteira que mais se aproxima de $(x, y)$ é $(a \cos (t), b \sin (t))$. Consequentemente, a distância entre $(x, y)$ e a fronteira da elipse é dada por

$$
\frac{s b}{a} \sqrt{b^{2} \cos (t)^{2}+a^{2} \sin (t)^{2}}
$$




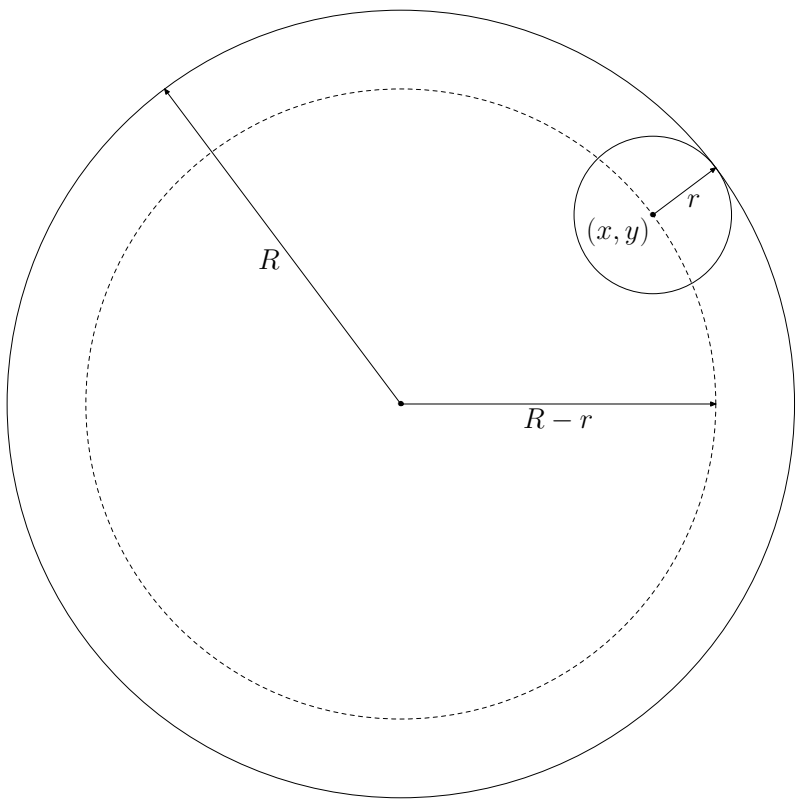

(a)

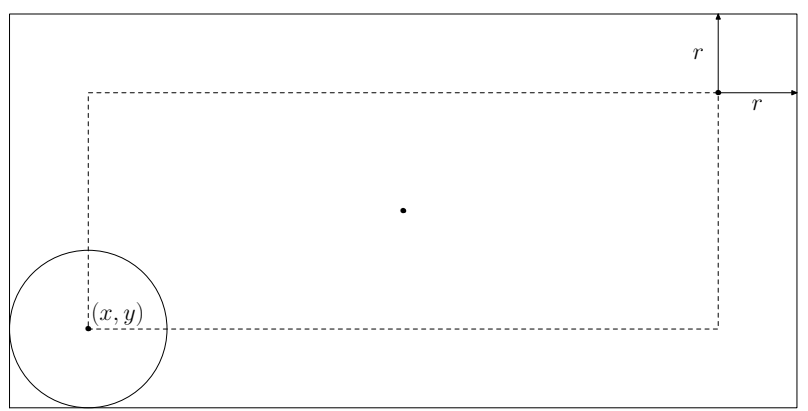

(b)

Figura 2.1: Empacotamento de círculos em círculo e em poliedros.

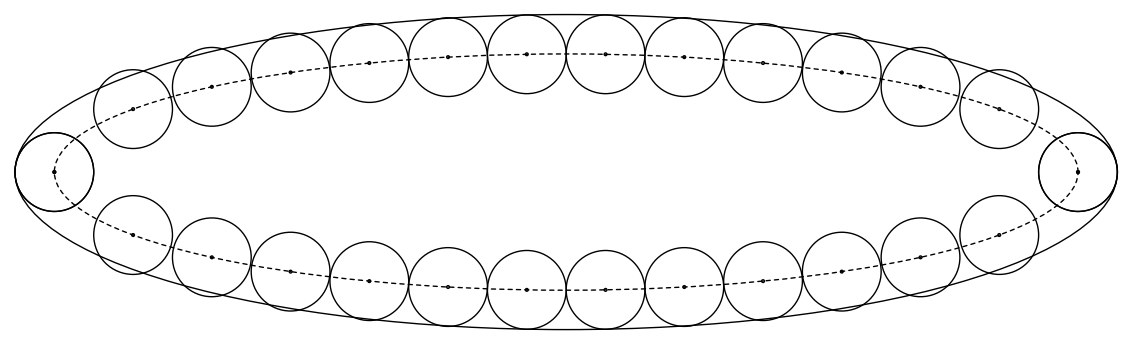

Figura 2.2: Tentativa de estabelecer uma restrição para que o contêiner elíptico contenha os itens. A elipse pontilhada satisfaz a distância do item de raio $r$ em $(0, b-r)$ à borda, mas não satisfaz necessariamente para outros itens de mesmo raio em outros pontos da elipse pontilhada.

Na Figura 2.3, mostramos graficamente como essa parametrização funciona. Observe que, para todos os pontos no segmento entre $\left(b^{2} / a-a, 0\right)$ e $\left(a-b^{2} / a, 0\right)$, existem dois pontos que satisfazem a menor distância à fronteira. Detalhes e propriedades teóricas sobre esta parametrização podem ser encontradas em [Cal12].

Uma parametrização diferente, baseada em curvas paralelas, foi utilizada em [Fri11] para resolver diferentes instâncias do problema de encontrar a elipse de menor área, que contém um número fixo de círculos unitários. As soluções exibidas em [Fri11] foram obtidas utilizando um pacote comercial de otimização global [Can11].

Pela parametrização $(2.1)$, temos que pontos $(x, y)$ no interior da elipse podem ser caracterizados por

$$
x=\left[1+(s-1)\left(b^{2} / a^{2}\right)\right] u, y=s v, 0 \leq s \leq 1,
$$

onde $(u / a)^{2}+(v / b)^{2}=1$ e $(u, v)$ é o ponto na fronteira da elipse que está mais próximo de $(x, y)$. Consequentemente, a distância de $(x, y)$ à fronteira é

$$
(1-s) \sqrt{\left(b^{2} / a^{2}\right)^{2} u^{2}+v^{2}}
$$




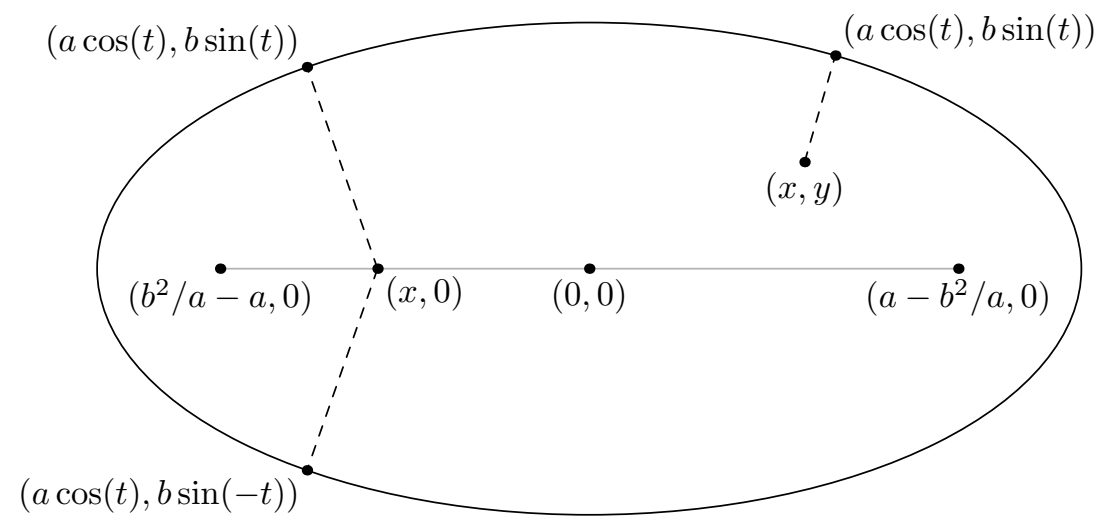

Figura 2.3: Parametrização definida em $(2.1)$ e, para um dado $(x, y)$, o ponto na fronteira que é mais próximo de $(x, y)$.

Considerando a parametrização descrita, quatro problemas relacionados ao empacotamento de círculos em elipses são formulados a seguir.

\subsubsection{Círculos dados em uma elipse fixa}

Considere o problema de encontrar $m$ discos não sobrepostos com raio $r_{i}, i=1, \ldots, m$, contidos na região definida pela elipse com $a \geq b$. Denotamos $\left(x_{i}, y_{i}\right)$ como o centro de cada círculo. Com o objetivo de obter a solução para o problema de empacotamento, devemos resolver o seguinte problema de viabilidade:

$$
\begin{gathered}
\left(u_{i} / a\right)^{2}+\left(v_{i} / b\right)^{2}=1, i=1, \ldots, m \\
\left(\frac{s_{i}-1}{r_{i}}\right)^{2}\left[\left(b^{2} / a^{2}\right)^{2} u_{i}^{2}+v_{i}^{2}\right] \geq 1, i=1, \ldots, m \\
\frac{1}{\left(r_{i}+r_{j}\right)^{2}}\left\{\left[\left(1+\left(s_{i}-1\right)\left(b^{2} / a^{2}\right)\right) u_{i}-\left(1+\left(s_{j}-1\right)\left(b^{2} / a^{2}\right)\right) u_{j}\right]^{2}+\left[s_{i} v_{i}-s_{j} v_{j}\right]^{2}\right\} \geq 1, \forall j>i \\
0 \leq s_{i} \leq 1, i=1, \ldots, m .
\end{gathered}
$$

Pela caracterização $(2.2)$, as coordenadas dos centros $\left(x_{i}, y_{i}\right)$ podem ser recuperadas usando

$$
x_{i}=\left[1+\left(s_{i}-1\right)\left(b^{2} / a^{2}\right)\right] u_{i}, \quad y_{i}=s_{i} v_{i}, \quad i=1, \ldots, m .
$$

Da mesma maneira, empregando (2.1), um problema de viabilidade também poderia ser formulado com as variáveis $t_{i}$ e $s_{i}, i=1, \ldots, m$. Damos preferência a (2.3-2.6) neste trabalho, uma vez que todas as restrições são formuladas como polinômios. As variáveis do problema não linear de viabilidade (2.3-2.6) são $\left(u_{i}, v_{i}\right), i=1, \ldots, m$ e $s_{i}, i=1, \ldots, m$. As restrições $(2.3),(2.4)$ e (2.5) definem $m$ restrições de igualdade e $m+m(m-1) / 2$ restrições de desigualdade; e (2.6) define $m$ restrições de caixas sobre as variáveis $s_{i}, i=1, \ldots, m$.

\subsubsection{Número máximo de círculos idênticos em uma elipse fixa}

Dado um número indefinido de círculos idênticos com raio $r_{i}=r$ para todo $i$ e uma elipse fixa, desejamos maximizar o número de círculos idênticos no interior da elipse. Se para $m$, o problema 
de viabilidade (2.3-2.6) tem uma solução, então a máxima quantidade de itens no interior da elipse é pelo menos $m$. A solução para este problema envolve uma sequência de problemas de viabilidade com $m$ crescente. Supondo que o limite inferior $m_{l b}$ no número de discos idênticos de raio $r$ que podem ser empacotados no interior da elipse é conhecido $\left(m_{l b}\right.$ igual a 0 pode ser uma escolha viável), o procedimento inicia tentando resolver o problema de viabilidade $(2.3-2.6)$ com $m=m_{l b}+1$. Se uma solução viável é encontrada, atribuímos $m \leftarrow m+1$ e tentamos novamente. O procedimento para quando $m$ é tal que uma solução viável para (2.3-2.6) não pode ser encontrada e a solução encontrada com $m^{*}=m-1$ é considerada a solução com maior quantidade de itens possível. Estratégias similares têm sido aplicadas em [BG10, BL10, BMNR06, BMR05] onde justificativas empíricas para o uso desse tipo de procedimento sequencial de incrementar $m$ um por um, em vez de outras estratégias como a bisseção, são dadas.

\subsubsection{Elipse de área mínima que contém um conjunto de círculos dados}

Desejamos encontrar a elipse de menor área que contém $m$ círculos com raio $r_{1}, \ldots, r_{m}$. Em princípio, o problema pode ser formulado como um problema de programação não linear

$$
\text { Minimizar } a b
$$

sujeito a (2.3-2.6), $a \geq 0$, e $b \geq 0$. Note que $a$ e $b$ são variáveis nesse problema, diferentemente dos anteriores, nos quais essas quantidades eram dadas. Mais ainda, dado que a validade da parametrização requer que $a \geq b$, é conveniente definir uma nova variável de folga $w \geq 0$ tal que $a=b+w$. Logo, as restrições de não negatividade $a \geq 0$ e $b \geq 0$ devem ser substituídas por

$$
b \geq 0 \text { e } w \geq 0
$$

e todas as ocorrências de $a$ na função objetivo (2.8) e nas restrições (2.3-2.6) devem ser substituídas por $b+w$.

\subsubsection{Bolas em um contêiner cilíndrico}

Os três problemas 2D descritos nas subseções anteriores podem ser estendidos para considerar a situação em 3D. Como exemplo, descrevemos o problema no qual temos $m$ esferas com raios $r_{1}, \ldots, r_{m}$ e desejamos empacotar essas $m$ esferas em um cilindro cuja base é uma elipse com semi-eixos $a \geq b$. Consideramos que o objetivo é minimizar a altura do contêiner.

Denotamos por $\left(x_{i}, y_{i}, z_{i}\right), i=1, \ldots, m$, os centros das esferas citadas. Como em $(2.7), x_{i}$ e $y_{i}$ são escritos como funções nas variáveis $u_{i}, v_{i}, s_{i}, i=1, \ldots, m$, tais que $\left(u_{i}, v_{i}, 0\right)$ é o ponto na fronteira do contêiner com base elíptica que está mais próximo de $\left(x_{i}, y_{i}, 0\right)$ - a projeção de $\left(x_{i}, y_{i}, z_{i}\right)$ no plano $x-y$. Logo, as restrições (2.3) e (2.6) fazem parte deste novo problema. A restrição (2.4) requer que a projeção de cada centro das esferas no plano $x-y$ esteja contido na elipse. Logo, a restrição (2.4) também está presente neste novo problema. Finalmente, a restrição de não sobreposição nas 3 dimensões é dada por

$\frac{1}{\left(r_{i}+r_{j}\right)^{2}}\left\{\left[\left(1+\left(s_{i}-1\right)\left(b^{2} / a^{2}\right)\right) u_{i}-\left(1+\left(s_{j}-1\right)\left(b^{2} / a^{2}\right)\right) u_{j}\right]^{2}+\left[s_{i} v_{i}-s_{j} v_{j}\right]^{2}+\left[z_{i}-z_{j}\right]^{2}\right\} \geq 1, \forall j>i$, 
As variáveis de altura $z_{i}$ são sujeitas a restrição

$$
0 \leq z_{i} \leq z_{\max }
$$

e a função objetivo do problema é

$$
\text { Minimizar } z_{\max }
$$

sujeito à $(2.3,2.4,2.6,2.10,2.11)$.

Esses quatro problemas são exemplos de muitas variações em 2 ou 3 dimensões de problemas de empacotamento envolvendo itens circulares ou esféricos em contêineres elípticos que podem ser formulados como problemas contínuos e diferenciáveis de programação não linear com a ajuda da parametrização (2.1). Outros exemplos incluem seções cônicas e interseções de elipses e podem ser encontrados em [Cal12]. 


\section{Capítulo 3}

\section{Procedimentos de otimização}

Todos os problemas apresentados no Capítulo 2 foram formulados em um arcabouço de Programação Não Linear. Eles têm a seguinte formulação matemática

$$
\text { Minimizar } f(x) \text { sujeito a } h(x)=0, g(x) \leq 0, \ell \leq x \leq u
$$

onde $f$ é uma função escalar diferenciável, $h$ e $g$ são funções vetoriais diferenciáveis e os limites para $x$ podem ser infinitos.

Nossa principal ferramenta para resolver (3.1) será o Algencan [ABMS07, ABMS08]. Algencan é um método para resolver problemas de programação não linear de larga escala que é periodicamente atualizado e publicamente disponível em http://www.ime.usp.br/ egbirgin/tango/. Algencan converge, sob condições razoáveis, a minimizadores locais do problema original. A convergência a minimizadores globais é perseguida nesta pesquisa por procedimentos que evocam a abordagem multistart de otimização global estocástica. Um procedimento diferente de globalização para o Algencan foi introduzido em [BFM10] com garantia de convergência a minimizadores globais, mas uma implementação para problemas de larga escala ainda não está disponível.

O problema de viabilidade (2.3-2.6), relacionado ao problema de maximizar o número de círculos idênticos no interior da elipse, é um caso particular de (3.1) em que a função objetivo é nula, i.e. pode ser escrito na forma

$$
\text { Ache } x \text { tal que } h(x)=0, g(x) \leq 0, \ell \leq x \leq u \text {, }
$$

onde $x=(u, v, s) \in \mathbb{R}^{3 m}$ a partir de agora. Um problema de viabilidade da forma (3.2) é equivalente ao problema de minimização em caixas

$$
\text { Minimizar } f(x) \text { sujeito a } \ell \leq x \leq u \text {, }
$$

onde

$$
f(x)=\|h(x)\|_{2}^{2}+\left\|g(x)_{+}\right\|_{2}^{2}
$$

e $\left[g(x)_{+}\right]_{i}=\max \left\{0, g_{i}(x)\right\}$. Se $f(\cdot)$ se anula no minimizador global $x^{*}$ de (3.3), então $x^{*}$ é uma solução viável para (3.2). Se, por outro lado, $f\left(x^{*}\right)>0$, então o problema (3.2) é inviável. Para o caso particular do problema de viabilidade (2.3-2.6), existem razões práticas para considerar a formulação de minimização em caixas (3.3) em vez da formulação como problema de viabilidade (3.2). 
Calcular $f(\cdot)$ envolve $O\left(\mathrm{~m}^{2}\right)$ restrições. Entretanto, dado que a soma envolvendo os valores dessas restrições é necessária, em vez dos valores individuais que seriam necessários para resolver (3.2) usando uma abordagem tradicional, uma estratégia desenvolvida para o problema "Nbody" [HE81] pode ser utilizada para reduzir a complexidade da avaliação de $f(\cdot)$. Essa estratégia, que será detalhada na seção seguinte, tem sido utilizada com sucesso pelo software de DM Packmol [MABM09, MM03] e está inteiramente descrita em [BS08], onde experimentos numéricos extensivos mostram a redução obtida no custo de avaliação $f(\cdot)$.

\subsection{Cálculo esparso da sobreposição}

Nesta seção, descrevemos a estratégia utilizada para o cálculo esparso da sobreposição. O conteúdo a seguir está fortemente baseado em [BS08].

No caso de itens circulares, a restrição de não sobreposição requer que os mesmos estejam a uma distância mínima igual à soma dos raios. Dados os itens $i$ e $j$, representados, respectivamente, pelas coordenadas $\left(x_{i}, y_{i}\right)$ e $\left(x_{j}, y_{j}\right)$ dos seus centros, $i=1,2, \ldots, m, j=1,2, \ldots, m$ e $i \neq j$, é necessário que a distância entre eles seja maior ou igual a $2 r$. Assim, a seguinte restrição deve ser satisfeita:

$$
\left(x_{i}-x_{j}\right)^{2}+\left(y_{i}-y_{j}\right)^{2} \geq(2 r)^{2}, \forall i<j,
$$

ou equivalentemente,

$$
\sum_{i<j} \max \left\{0,(2 r)^{2}-\left(x_{i}-x_{j}\right)^{2}-\left(y_{i}-y_{j}\right)^{2}\right\}^{2}=0
$$

Tanto (3.5) quanto (3.6) estão relacionadas à distância dos centros entre os $m(m-1) / 2$ pares de itens. Os quadrados em (3.5) e (3.6) são utilizados para torná-las diferenciáveis. Claramente, (3.5) e (3.6) são equivalentes e qualquer conjunto de itens que as satisfaçam não possui sobreposição entre os itens.

Na prática, (3.6) tem grande vantagem sobre (3.5). Nem todas as $O\left(m^{2}\right)$ distâncias necessitam ser calculadas para avaliar o termo à esquerda em (3.6). Basicamente, se dois itens circulares $k_{1} \mathrm{e}$ $k_{2}$ estão longe um do outro, a contribuição do termo $\max \left\{0,(2 r)^{2}-\left(x_{k_{1}}-x_{k_{2}}\right)^{2}-\left(y_{k_{1}}-y_{k_{2}}\right)^{2}\right\}^{2}$ ao somatório em (3.6) é nula. De fato, se os itens estiverem mais ou menos bem distribuídos, o número de pares que contribuem no somatório em (3.6) é $O(m)$. Mais ainda, esses pares podem ser identificados em $O(m)$ operações (para mais detalhes veja [BS08]).

Consideramos um particionamento do contêiner em regiões, de tal forma que itens em regiões não adjacentes não se sobreponham. É fácil ver que uma região quadrada de lado $\delta=2 r$ define um particionamento com essa propriedade quando estamos lidando com itens circulares idênticos de raio $r$ (veja Figura 3.1). Para um dado item, somente é necessário computar as distâncias do seu centro aos centros dos itens em regiões adjacentes.

O particionamento também dependerá da forma do contêiner. Considerando o contêiner elíptico, o número total de regiões necessárias para cobri-lo totalmente seria $N_{r e g} A * N_{\text {reg_B }}$, onde $N_{\text {reg_A }}=$ $\lceil 2 a / \delta\rceil$ e $N_{\text {reg_B }}=\lceil 2 b / \delta\rceil$.

Dado $c_{i}=\left(x_{i}, y_{i}\right) \in \mathbb{R}^{2}, i=1,2, \ldots, m$, a região na qual $c$ está contido pode ser computada em tempo constante:

$$
\operatorname{Reg}\left(c_{i}\right)=\left(P_{a}\left(x_{i}\right), P_{b}\left(y_{i}\right)\right)
$$




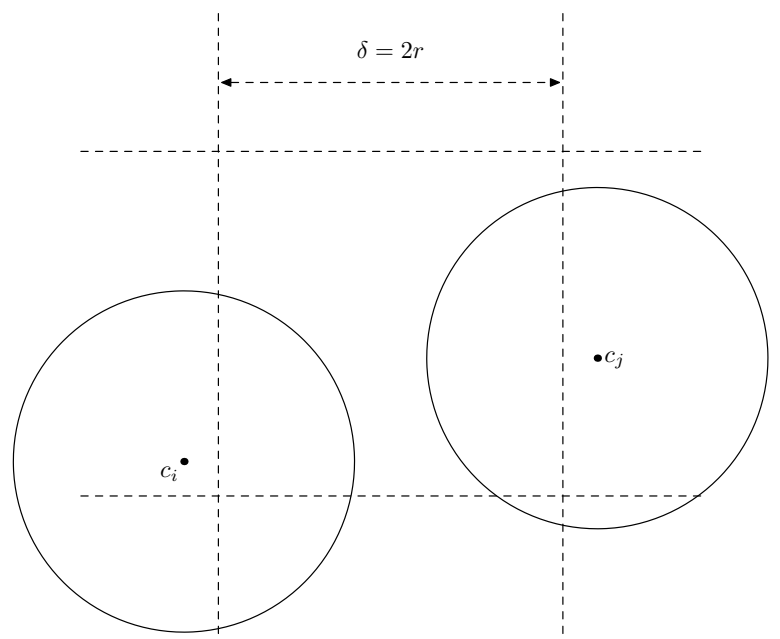

Figura 3.1: Uma partição para itens circulares de raio $r$, não há sobreposição de itens em regiões não adjacentes.

onde

$$
\begin{aligned}
& P_{a}\left(x_{i}\right)=\max \left\{0, \min \left\{\left\lfloor x_{i} / \delta\right\rfloor, N_{\text {reg_A }}+1\right\}\right\}, \\
& P_{b}\left(y_{i}\right)=\max \left\{0, \min \left\{\left\lfloor y_{i} / \delta\right\rfloor, N_{\text {reg_B }}+1\right\}\right\} .
\end{aligned}
$$

Dessa maneira, a avaliação da restrição (3.6) inicia com uma estrutura de dados vazia que representa as regiões. Cada região tem uma lista de itens, cujos centros estão contidos nela. Usa-se uma matriz com $\left(N_{\text {reg_A }}+2\right) *\left(N_{\text {reg_B }} B+2\right)$ elementos com ponteiros para essas listas. Também utiliza-se uma lista para as regiões não vazias (regiões que contenham pelo menos um item) (veja a Figura 3.2).

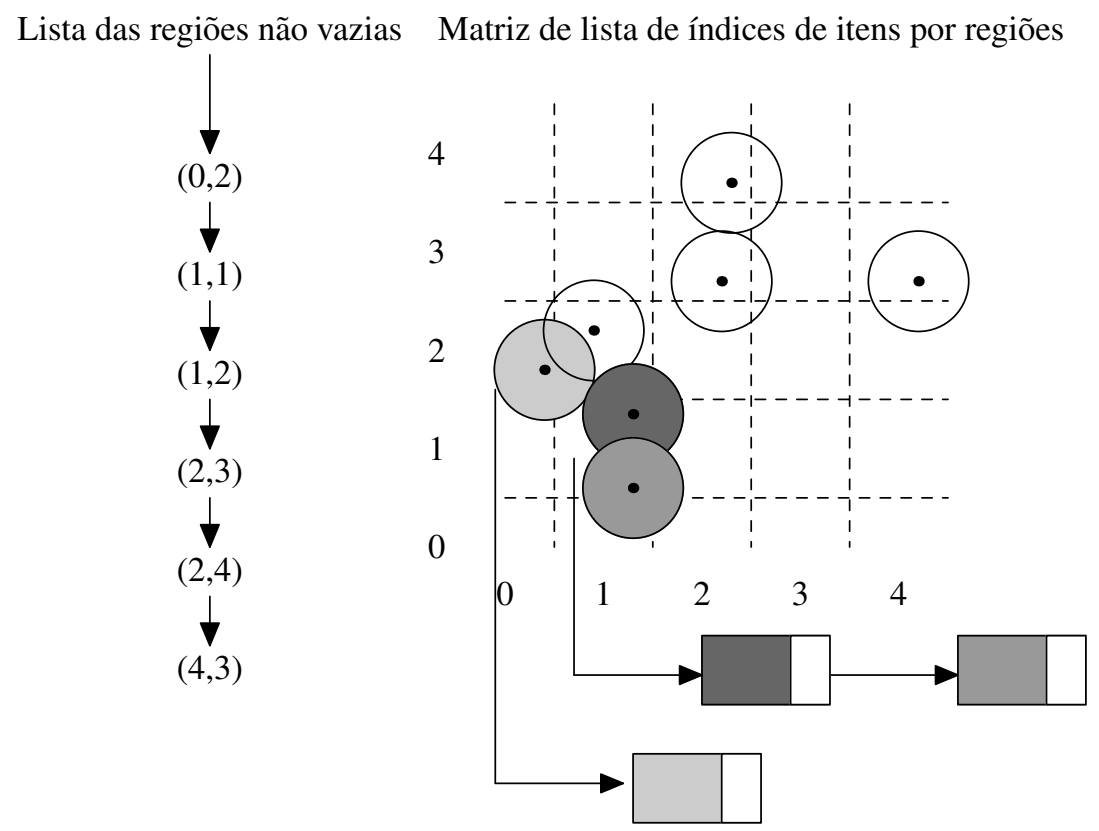

Figura 3.2: Estrutura de dados para avaliação de sobreposição. Cada partição contém uma lista de itens contidos. Também existe uma lista de regiões não vazias.

Dados os centros do itens $i=1, \ldots, m$, os passos utilizados para a avaliação da restrição (3.6) são dados a seguir: 


\section{Algoritmo 3.1}

Passo 0: sum $\leftarrow 0$.

Passo 1: Para cada $i=1, \ldots, m$ faça:

Passo 1.1: Determine a região $\operatorname{Reg}\left(c_{i}\right)$ dada por (3.7)-(3.8).

Passo 1.2: Adicione $i$ à lista de itens com centros na $\operatorname{Reg}\left(c_{i}\right)$.

Passo 1.3: Se for o primeiro item em $\operatorname{Reg}\left(c_{i}\right)$, coloque $\operatorname{Reg}\left(c_{i}\right)$ na lista de regiões não vazias.

Passo 2: Para cada região não vazia $R$ e para cada item $i$ em $R$ faça:

Passo 2.1: Para cada item $j$ em $R$ ou nas regiões adjacentes à $R$ faça:

Passo 2.1.1: Calcule sum $\leftarrow$ sum $+\max \left\{0,(2 r)^{2}-\left(x_{i}-x_{j}\right)^{2}-\left(y_{i}-y_{j}\right)^{2}\right\}$.

Passo 3: Limpe as estruturas de dados e devolva sum.

Supondo que o número de itens em cada região é constante quando próximo da solução (quer dizer, não depende de $m$ ), então a complexidade de todo processo seria $O(m)$. A consideração do número constante de itens por região é razoável, uma vez que, quando o método se aproxima da solução, os itens se encontram mais ou menos bem distribuídos.A estratégia está completamente descrita em [BS08], com testes numéricos exaustivos que mostram a eficácia da abordagem. Também tem sido usada com sucesso em problemas de conformação molecular em que o número de itens pode ser muito grande [MABM09, MM03]. 


\section{Capítulo 4}

\section{Experimentos computacionais}

Neste Capítulo, reportamos experimentos numéricos extensivos para o problema de empacotamento da máxima quantidade de discos unitários possíveis em uma elipse. Todos os testes foram conduzidos em um InTEL XEON com 2.67GHz e 8GB de memória RAM, rodando o sistema operacional GNU/LinUX. O programa, inteiramente implementado em ForTRAN 77, foi compilado pelo G77 ForTrAn, compilador do GCC, com a diretiva de otimização - 04 habilitada.

Como foi mencionado no capítulo anterior, o método de solução consiste em resolver uma sequência de problemas de minimização em caixas (3.3) para valores crescentes de $m=m_{l b}+m_{+}$, $m_{+}=1,2, \ldots$ Neste caso, o problema (3.2) toma a forma

$$
\begin{aligned}
h_{i}^{\xi}(u, v, s) & =0, \quad i=1, \ldots, m \\
g_{i}^{\xi}(u, v, s) & \leq 0, \quad i=1, \ldots, m \\
\kappa_{i j}^{\xi}(u, v, s) & \leq 0, \quad i=1, \ldots, m, j=i+1, \ldots, m \\
0 \leq s_{i} & \leq 1, \quad i=1, \ldots, m
\end{aligned}
$$

onde

$$
\begin{aligned}
h_{i}^{\xi}(u, v, s) & =1-\left[\left(u_{i} / a\right)^{2}+\left(v_{i} / b\right)^{2}\right] \\
g_{i}^{\xi}(u, v, s) & =1-\left(\frac{s_{i}-1}{r}\right)^{2}\left[\left(b^{2} / a^{2}\right)^{2} u_{i}^{2}+v_{i}^{2}\right] \\
\kappa_{i j}^{\xi}(u, v, s) & =1-\frac{1}{4 r^{2}}\left[\left\{\left(1+\left(s_{i}-1\right)\left(b^{2} / a^{2}\right)\right) u_{i}-\left(1+\left(s_{j}-1\right)\left(b^{2} / a^{2}\right)\right) u_{j}\right\}^{2}+\left\{s_{i} v_{i}-s_{j} v_{j}\right\}^{2}\right]
\end{aligned}
$$

e $\xi=(a, b, r, m)$ é utilizado para ressaltar a dependência nos parâmetros do problema. Entretanto, o problema (3.3) toma a forma

$$
\operatorname{Min} f^{\xi}(u, v, s) \text { sujeito a } 0 \leq s \leq 1
$$

onde

$$
f^{\xi}(u, v, s)=\sum_{i=1}^{m} h_{i}^{\xi}(u, v, s)^{2}+\sum_{i=1}^{m} g_{i}^{\xi}(u, v, s)_{+}^{2}+\sum_{i=1}^{m} \sum_{j=i+1}^{m} \kappa_{i j}^{\xi}(u, v, s)_{+}^{2} .
$$

Se, para um dado $m$, um minimizador global de (4.3) com função objetivo nula é encontrado, o valor de $m$ é incrementado e o processo continua. Caso contrário, continuamos tentando com o valor de $m$ até que o limite de tempo de CPU imposto seja atingido. Essa estratégia se baseia em dois fatos: (a) o procedimento para encontrar minimizadores globais de (4.3) utiliza um método estocástico de otimização global baseado no multistart e (b) somos capazes de reconhecer que um 
minimizador global foi encontrado somente se o mínimo global da instância a ser resolvida for zero. O procedimento está descrito no Algoritmo 4.1

Algoritmo 4.1 Sejam $a \geq b>0$ e $r>0$ constantes dadas.

Passo 1. Calcule o limite inferior $m_{l b}$, i.e. um valor $m_{l b} \geq 0$ tal que $\left(u^{*}, v^{*}, s^{*}\right) \in \mathbb{R}^{3 m_{l b}}$ com $f^{\xi}\left(u^{*}, v^{*}, s^{*}\right)=0$ seja conhecido (note que $m_{l b}=0$ é uma escolha válida). Faça $m_{+} \leftarrow 1 \mathrm{e}$ $m \leftarrow m_{l b}+m_{+}$.

Passo 2. Se o limite de tempo é atingido, pare declarando que $m^{*}=m-1$ itens foram empacotados.

Passo 3. Calcule um ponto inicial aleatório $\left(u_{\text {ini }}, v_{\text {ini }}, s_{\text {ini }}\right)$ para o problema (4.3).

Passo 4. Encontre um ponto estacionário $\left(u^{*}, v^{*}, s^{*}\right)$ de (4.3) iniciando a partir de $\left(u_{\text {ini }}, v_{\text {ini }}, s_{\text {ini }}\right)$.

Passo 5. Se $f^{\xi}\left(u^{*}, v^{*}, s^{*}\right)$ se anula, faça $m_{+} \leftarrow m_{+}+1, m \leftarrow m_{l b}+m_{+}$.

Passo 6. Vá para o Passo 2.

Nas seguintes seções, descreveremos cada passo do Algoritmo 4.1 em detalhe.

\subsection{Calculando o limite inferior $m_{l b}$}

Em conexão com a estratégia de otimização para o empacotamento do número máximo de círculos idênticos possíveis no interior de uma elipse, desenvolvemos um limitante inferior baseado no reticulado hexagonal (veja a Figura 4.1). O empacotamento de discos no plano (infinito) se dá com maior densidade em uma distribuição no reticulado hexagonal, com densidade de $\eta_{h}=\frac{1}{6} \pi \sqrt{3} \approx$ 0.906899682 [Wei11].

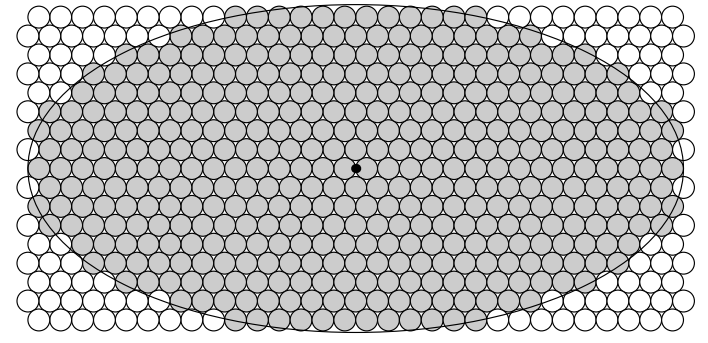

(a) $\mathcal{L}(r, 0)$

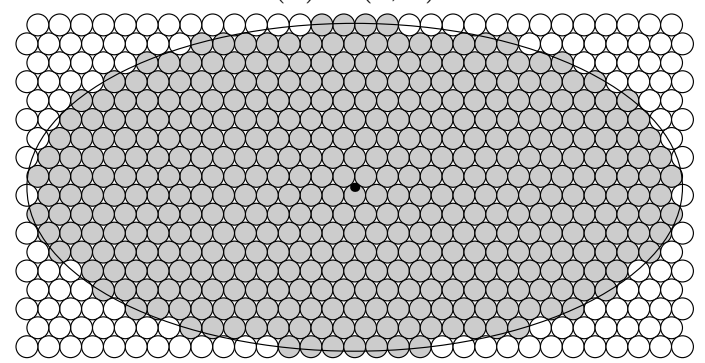

(c) $\mathcal{L}(r, r)$

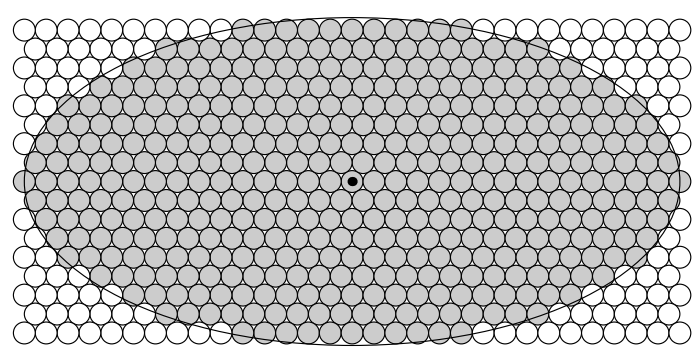

(b) $\mathcal{L}(0,0)$

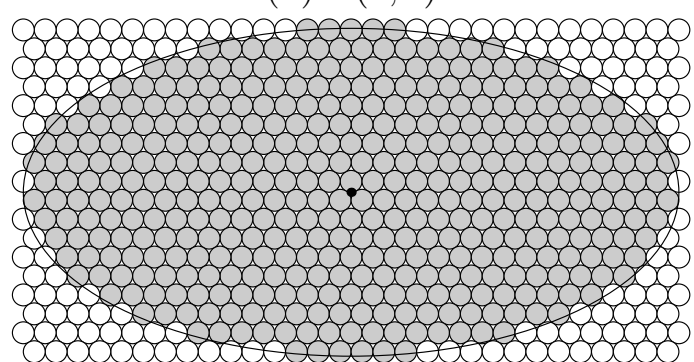

(d) $\mathcal{L}(0, r)$

Figura 4.1: (a-d) Reticulados $\mathcal{L}(r, 0), \mathcal{L}(0,0), \mathcal{L}(r, r), \mathcal{L}(0, r)$, respectivamente, ilustrando como eles podem ser utilizadas para construir limites inferiores para instâncias com $a=30, b=15$, e $r=1$. Os círculos brancos são os círculos do reticulado cujos centros estão fora da elipse. Os discos cinza-claro são aqueles que possuem o centro no interior do elipse e podem contribuir para o limitante inferior. 
Dado um ponto $(\bar{x}, \bar{y})^{T} \in \mathbb{R}^{2}$, denotamos $\mathcal{L}(\bar{x}, \bar{y})$ como o conjunto infinito de pontos no reticulado hexagonal cujas linhas são paralelas ao eixo Cartesiano horizontal e tal que $(\bar{x}, \bar{y})^{T} \in \mathcal{L}(\bar{x}, \bar{y})$. A Figura 4.1(a-d) ilustra os reticulados $\mathcal{L}(r, 0), \mathcal{L}(0,0), \mathcal{L}(r, r), \mathcal{L}(0, r)$, com $r=1$, respectivamente. A computação do limite inferior $m_{l b}$ para o número de círculos idênticos que podem ser empacotados em uma elipse dada por $a \geq b$, inicia considerando o reticulado $\mathcal{L}(\bar{x}, \bar{y})$ e calculando o conjunto finito de pontos $P(\bar{x}, \bar{y})=\mathcal{L}(\bar{x}, \bar{y}) \cap\left\{(x, y)^{T} \in \mathbb{R}^{2} \mid(x / a)^{2}+(y / b)^{2} \leq 1\right\}$. Esses são os centros dos círculos cinza-claro na Figura 4.1, que são "candidatos" a estarem dentro da elipse. Para cada $\left(x_{i}, y_{i}\right)^{T} \in P(\bar{x}, \bar{y})$, consideramos duas formas de determinar se o círculo de raio $r$ com centro $\left(x_{i}, y_{i}\right)^{T}$ está dentro da elipse ou não. A primeira forma consiste em aproximar a elipse por um poliedro totalmente contido na elipse. Depois é fácil checar se um dado círculo está dentro do poliedro ou não, checando se o seu centro está dentro do poliedro e a distância pelo menos $r$ da borda. Na Figura 4.2, ilustramos a estratégia considerando poliedros com um número variável de restrições lineares para o reticulado $\mathcal{L}(r, 0)$. Os círculos cinza-escuro são aqueles com centro no interior do poliedro tais que o círculo não está inteiramente contido no mesmo. O limite inferior é dado pelo número de círculos cinza-claro.

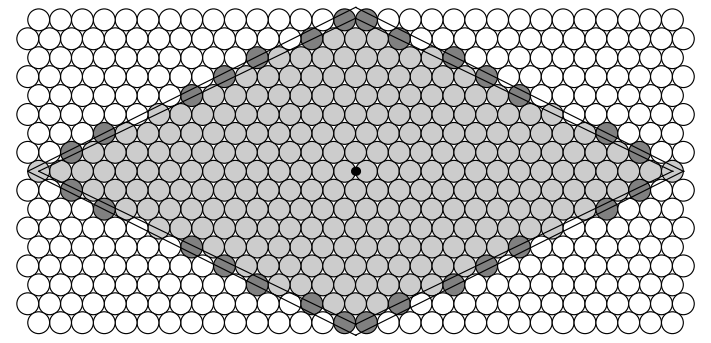

(a) Poliedro com 4 lados

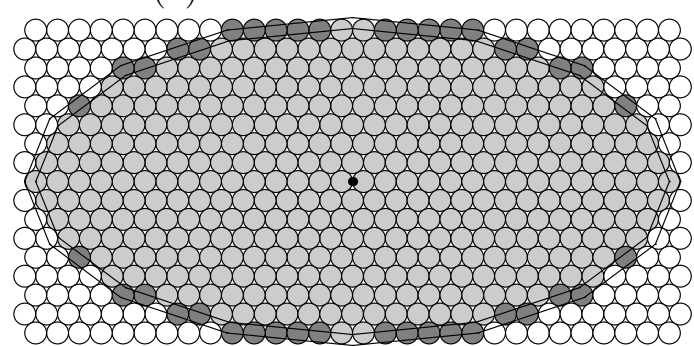

(c) Poliedro com 16 lados

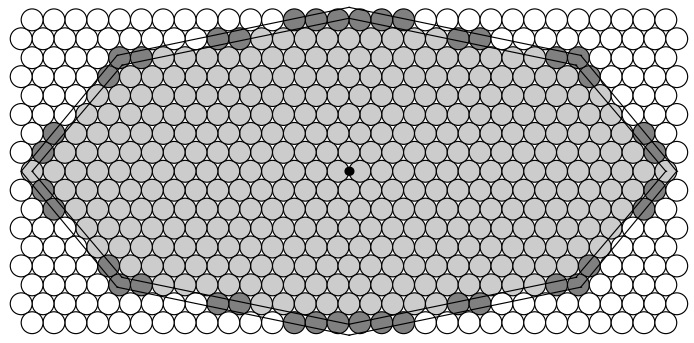

(b) Poliedro com 8 lados

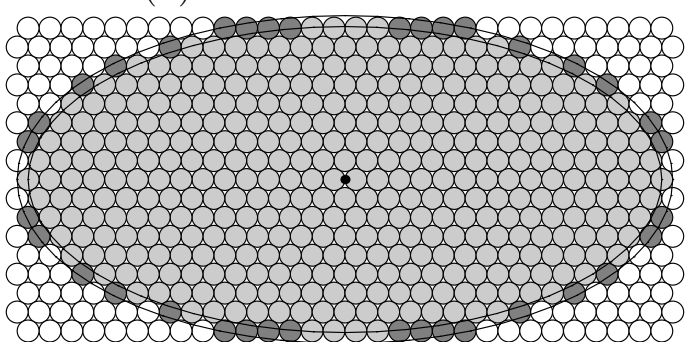

(d) Poliedro com 64 lados

Figura 4.2: Quatro poliedros (a-d) que aproximam a elipse com $a=30, b=15$, e $r=1$. Cada um dos poliedros determina respectivamente os seguintes limites inferiores: 234,330,358 e 374. Para estas figuras o reticulado utilizado foi $\mathcal{L}(r, 0)$.

A segunda forma de determinar se um círculo com centro $\left(x_{i}, y_{i}\right)$ está dentro da elipse consiste em procurar $u_{i}, v_{i}$, e $0 \leq s_{i} \leq 1$, tais que

$$
\begin{aligned}
{\left[1+\left(s_{i}-1\right)\left(b^{2} / a^{2}\right)\right] u_{i} } & =x_{i}, \\
s_{i} v_{i} & =y_{i} \\
\left(u_{i} / a\right)^{2}+\left(v_{i} / b\right)^{2} & =1, \\
\left(\left(s_{i}-1\right) / r\right)^{2}\left[\left(b^{2} / a^{2}\right)^{2} u_{i}^{2}+v_{i}^{2}\right] & \geq 1 .
\end{aligned}
$$

Esse problema de viabilidade com três variáveis é resolvido com o Algencan. Na Figura 4.3, círculos cinza-escuro são aqueles com centro no interior da elipse tais que o círculo não está inteiramente 


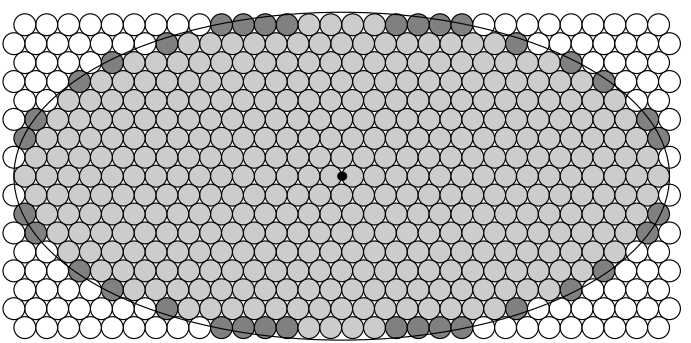

(a) $\mathcal{L}(r, 0)$

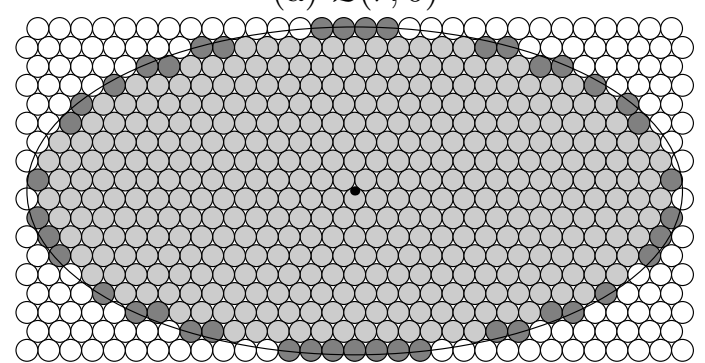

(c) $\mathcal{L}(r, r)$

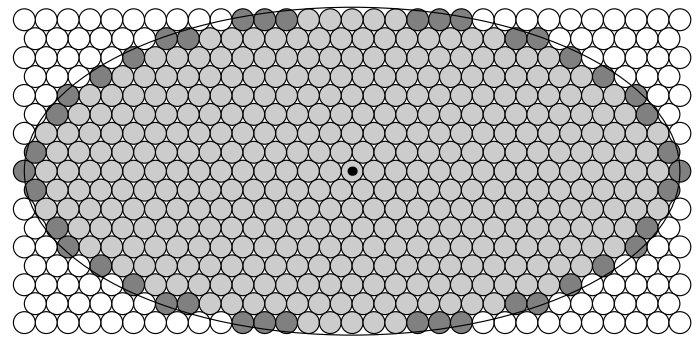

(b) $\mathcal{L}(0,0)$

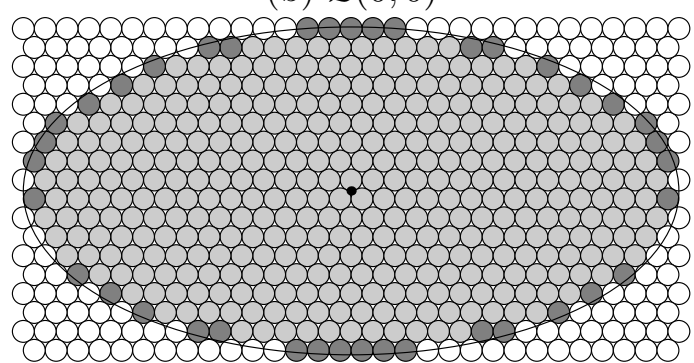

(d) $\mathcal{L}(0, r)$

Figura 4.3: (a-d) Reticulados $\mathcal{L}(r, 0), \mathcal{L}(0,0), \mathcal{L}(r, r), \mathcal{L}(0, r)$, respectivamente, ilustrando como elas podem ser utilizadas para construir limites inferiores para instâncias com $a=30, b=15$, e $r=1$. Os círculos brancos são os círculos do reticulado cujos centros estão fora da elipse. Os discos cinza-escuro são aquele cujos centros estão dentro da elipse, mas que não estão completamente contidos na elipse. Os discos cinza-claro são aqueles que estão dentro da elipse e contribuem para o limitante inferior. As quatro reticulados fornecem os limites inferiores $374,365,368$ e 366 , respectivamente, i.e. $\mathcal{L}(r, 0)$ fornece o melhor limite inferior para essa instância.

contido na elipse. O número de círculos cinza-claro é o limite inferior dado pelo reticulado $\mathcal{L}(\bar{x}, \bar{y})$. As Figuras 4.2(d) e 4.3(a) ilustram o cálculo do limitante baseado no reticulado $\mathcal{L}(r, 0)$ utilizando as estratégias do poliedro e do sistema não linear, respectivamente. Note que com o número de lados igual a 64 na estratégia do poliedro ambas as estratégias coincidem no cálculo do limitante $m_{l b}=374$. Independentemente disso, neste trabalho optamos pela segunda estratégia já que esta, diferentemente da primeira, fornece valores para as variáveis $u_{i}, v_{i}$ e $s_{i}$ que podem ser utilizados como chute inicial no processo posterior de otimização.

Reticulados diferentes podem fornecer diferentes limites inferiores. Por exemplo, reticulados na Figura 4.3(a-d) fornecem limites inferiores iguais a 374, 365, 368 e 366, respectivamente, para instância com $a=2 b=30$ e $r=1$. Neste trabalho, para cada elipse dada por $a \geq b$ e círculos com raio $r$, o limite inferior $m_{l b}$ é calculado como o maior limite inferior dado por um dos quatro reticulados representados na Figura 4.3. A Tabela 4.1 mostra alguns resultados numéricos. Pode ser observado que: (a) nenhum dos quatro reticulados considerados foi melhor que os outros, no sentido de dar o melhor limite inferior para todos os casos; (b) quanto maior a elipse, melhor o limite inferior (se considerarmos que uma densidade alta é um indicativo de que o limite inferior está próximo da solução ótima); e (c) quanto maior a razão $a / b$, mais pobre será o limite inferior. Por (b) e (c), vemos que a estratégia descrita fornece limites inferiores "razoáveis" para elipses "grandes" e "largas", deixando mais espaço para facilmente obter soluções melhores para instâncias com elipses "pequenas" ou "finas". Todo processo de computação do limite inferior como descrito levou menos que 0.1 segundo de tempo de CPU no ambiente computacional descrito no início do capítulo. 


\begin{tabular}{|c|c|c|c|c|c|c|c|c|c|c|c|}
\hline \multicolumn{5}{|c|}{ Instância } & \multicolumn{4}{|c|}{ Limite dado } & \multicolumn{2}{|c|}{ Melhor $m_{l b}$} & \multirow{2}{*}{$\begin{array}{l}\text { Tempo } \\
\text { (em s.) }\end{array}$} \\
\hline & Nome & $a$ & $b$ & $a / b$ & $\mathcal{L}(r, 0)$ & $\mathcal{L}(0,0)$ & $\mathcal{L}(r, r)$ & $\mathcal{L}(0, r)$ & $m_{l b}$ & Densidade & \\
\hline \multirow{14}{*}{ 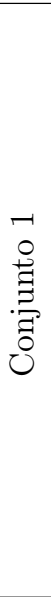 } & $\mathrm{s} 1 \mathrm{i01}$ & 4 & 2 & 2 & 4 & 3 & 1 & 3 & 4 & 0.5000 & 0.02 \\
\hline & s1i02 & 6 & 3 & 2 & 12 & 9 & 9 & 9 & 12 & 0.6667 & 0.03 \\
\hline & s1i03 & 8 & 4 & 2 & 18 & 19 & 20 & 18 & 20 & 0.6250 & 0.03 \\
\hline & s1i04 & 10 & 5 & 2 & 36 & 35 & 30 & 30 & 36 & 0.7200 & 0.05 \\
\hline & s1i05 & 12 & 6 & 2 & 50 & 45 & 49 & 51 & 51 & 0.7083 & 0.06 \\
\hline & s1i06 & 14 & 7 & 2 & 74 & 71 & 67 & 68 & 74 & 0.7551 & 0.10 \\
\hline & s1i07 & 16 & 8 & 2 & 92 & 95 & 94 & 92 & 95 & 0.7422 & 0.12 \\
\hline & s1i08 & 18 & 9 & 2 & 126 & 121 & 123 & 121 & 126 & 0.7778 & 0.17 \\
\hline & s1i09 & 20 & 10 & 2 & 158 & 159 & 151 & 155 & 159 & 0.7950 & 0.21 \\
\hline & s1i10 & 22 & 11 & 2 & 192 & 185 & 190 & 192 & 192 & 0.7934 & 0.24 \\
\hline & s1i11 & 24 & 12 & 2 & 238 & 225 & 226 & 222 & 238 & 0.8264 & 0.26 \\
\hline & s1i12 & 26 & 13 & 2 & 268 & 267 & 273 & 273 & 273 & 0.8077 & 0.32 \\
\hline & s1i13 & 28 & 14 & 2 & 320 & 317 & 311 & 314 & 320 & 0.8163 & 0.43 \\
\hline & s1i14 & 30 & 15 & 2 & 374 & 365 & 368 & 366 & 374 & 0.8311 & 0.47 \\
\hline \multirow{12}{*}{ 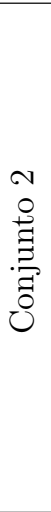 } & s2i01 & 32.1429 & 14 & 2.2959 & 368 & 369 & 363 & 364 & 369 & 0.8200 & 0.45 \\
\hline & s2i02 & 34.6154 & 13 & 2.6627 & 360 & 359 & 365 & 367 & 367 & 0.8156 & 0.45 \\
\hline & s2i03 & 37.5000 & 12 & 3.1250 & 362 & 363 & 354 & 360 & 363 & 0.8067 & 0.45 \\
\hline & s2i04 & 40.9091 & 11 & 3.7190 & 354 & 359 & 364 & 360 & 364 & 0.8089 & 0.48 \\
\hline & s2i05 & 45.0000 & 10 & 4.5000 & 358 & 361 & 353 & 353 & 361 & 0.8022 & 0.48 \\
\hline & s2i06 & 50.0000 & 9 & 5.5556 & 354 & 349 & 349 & 349 & 354 & 0.7867 & 0.47 \\
\hline & s2i07 & 56.2500 & 8 & 7.0313 & 340 & 339 & 346 & 346 & 346 & 0.7689 & 0.53 \\
\hline & s2i08 & 64.2857 & 7 & 9.1837 & 340 & 341 & 329 & 326 & 341 & 0.7578 & 0.50 \\
\hline & s2i09 & 75.0000 & 6 & 12.5000 & 308 & 307 & 329 & 331 & 331 & 0.7356 & 0.46 \\
\hline & s2i10 & 90.0000 & 5 & 18.0000 & 318 & 323 & 290 & 292 & 323 & 0.7178 & 0.51 \\
\hline & s2i11 & 112.5000 & 4 & 28.1250 & 274 & 273 & 296 & 296 & 296 & 0.6578 & 0.53 \\
\hline & s2i12 & 150.0000 & 3 & 50.0000 & 264 & 265 & 235 & 233 & 265 & 0.5889 & 0.56 \\
\hline \multirow{6}{*}{ 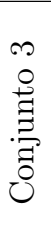 } & s3i01 & 100.0000 & 2.0000 & 50.0000 & 86 & 87 & 49 & 51 & 87 & 0.4350 & 0.28 \\
\hline & s3i02 & 75.0000 & 1.5000 & 50.0000 & 56 & 55 & 0 & 0 & 56 & 0.4978 & 0.15 \\
\hline & s3i03 & 75.0000 & 2.6667 & 28.1250 & 70 & 69 & 107 & 107 & 107 & 0.5350 & 0.24 \\
\hline & s3i04 & 56.2500 & 2.0000 & 28.1250 & 48 & 49 & 29 & 29 & 49 & 0.4356 & 0.15 \\
\hline & s3i05 & 60.0000 & 3.3333 & 18.0000 & 128 & 125 & 99 & 99 & 128 & 0.6400 & 0.25 \\
\hline & s3i06 & 45.0000 & 2.5000 & 18.0000 & 42 & 41 & 59 & 59 & 59 & 0.5244 & 0.14 \\
\hline
\end{tabular}

Tabela 4.1: O conjunto 1 corresponde à elipses com área crescente e razão $a / b$ constante, o conjunto 2 corresponde à elipses com área constante (igual a área da maior elipse do conjunto 1) e razão $a / b$ crescente, e o conjunto 3 corresponde à elipses mais "finas" com área relativamente grande e razão $a / b$ grande. 


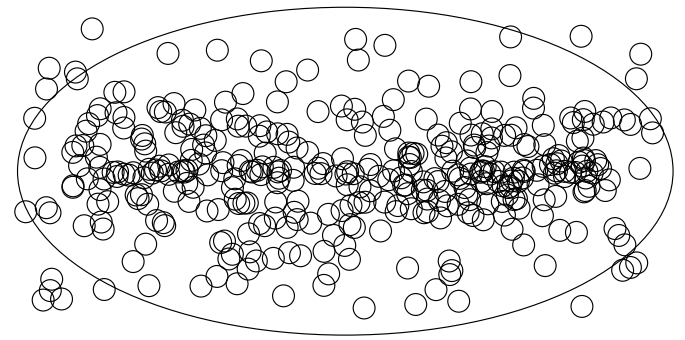

(a) R1

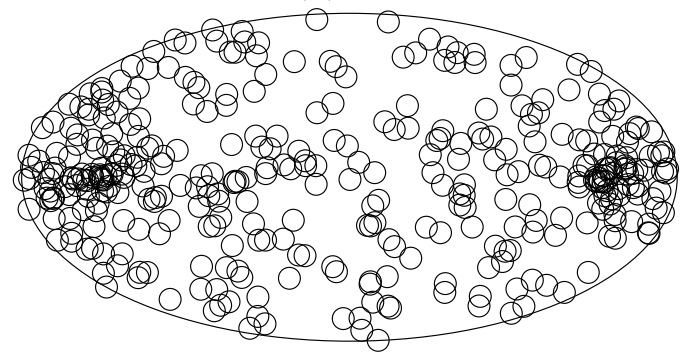

(c) R3

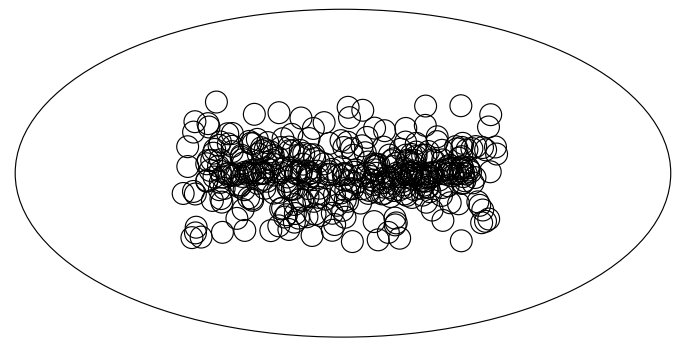

(b) R2

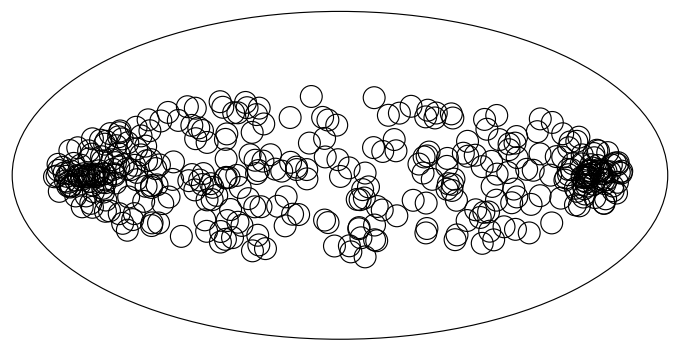

(d) R4

Figura 4.4: (a-d) Tipo de pontos iniciais aleatórios R1, R2, R3 e R4, respectivamente, para tentativa de empacotar $m=375$ círculos (um a mais que o número de círculos dado pelo limite inferior fornecido pelo reticulado) para instâncias $a=30, b=15$ e $r=1$.

\section{$4.2 \quad$ Multistart simples}

Nesta seção, descrevemos diferentes alternativas para construção do ponto inicial, necessário para encontrar um ponto estacionário de (4.3) no Passo 4 do Algoritmo 4.1. Consideramos inicialmente pontos iniciais aleatórios triviais que não fazem uso de nenhuma solução obtida anteriormente. Os quatro tipos considerados de pontos iniciais diferem no domínio em que as variáveis dos círculos são geradas.

- Tipo R1: itens são sorteados com $\left(u_{i}, v_{i}, s_{i}\right)^{T} \in[-a,-b, 0] \times[a, b, 1]$,

- Tipo R2: itens são sorteados com $\left(u_{i}, v_{i}, s_{i}\right)^{T} \in\left[-\frac{1}{2} a,-\frac{1}{2} b, 0\right] \times\left[\frac{1}{2} a, \frac{1}{2} b, 1\right]$,

- Tipo R3: itens são sorteados com $\left(u_{i}, v_{i}\right)$ tais que $\left(u_{i} / 2\right)^{2}+\left(v_{i} / 2\right)^{2}=1$ e $s_{i} \in[0,1]$,

- Tipo R4: itens são sorteados com $\left(u_{i}, v_{i}\right)$ tais que $\left(u_{i} / 2\right)^{2}+\left(v_{i} / 2\right)^{2}=1$ e $s_{i} \in\left[0, \frac{1}{2}\right]$.

Esses quatro tipos de pontos iniciais estão representados na Figura 4.4. Como bem sabemos, nenhum desses quatro tipos de estratégias fornecem uma distribuição uniforme dos círculos. Se necessário, uma distribuição uniforme dos círculos poderia ser gerada distribuindo aleatoriamente $\left(x_{i}, y_{i}\right) \in$ $[-a,-b] \times[a, b]$ e tendo o custo de resolver o problema de viabilidade com três variáveis (4.5) para encontrar $\left(u_{i}, v_{i}, s_{i}\right)$.

O efeito do uso dos quatro tipos descritos para pontos iniciais foi avaliado rodando o Algoritmo 4.1. No Passo 1, o limite inferior $m_{l b}$ foi computado como descrito na Seção 4.1. No Passo 2, arbitrariamente fixamos o limite de Tempo de CPU em 6 horas. No Passo 3, as quatro diferentes estratégias R1, R2, R3 e R4 foram consideradas para a geração do ponto inicial aleatório. No Passo 4, do Algoritmo 4.1, pontos estacionários aproximados, candidatos a minimizadores globais do problema de minimização em caixas (4.3), são computados usando Algencan [ABMS07, ABMS08]. Os parâmetros padrões do Algencan foram utilizados, i.e. os pontos estacionários aproximados $\left(u^{*}, v^{*}, s^{*}\right)$ 
satisfazem à condição

$$
\left\|P_{\Omega}\left[\left(u^{*}, v^{*}, s^{*}\right)-\nabla f^{\xi}\left(u^{*}, v^{*}, s^{*}\right)\right]-\left(u^{*}, v^{*}, s^{*}\right)\right\|_{\infty} \leq 10^{-8},
$$

onde $\Omega=\left\{(u, v, s) \in \mathbb{R}^{3 m} \mid 0 \leq s \leq 1\right\}$ representa a região viável do problema (4.3) e $P_{\Omega}(u, v, s)$ denota a projeção ortogonal Euclidiana de $(u, v, s)$ no conjunto convexo $\Omega$. Um ponto estacionário aproximado $\left(u^{*}, v^{*}, s^{*}\right)$ é considerado um minimizador global de (4.3) (Passo 5) quando satisfaz

$$
\max \left\{\left\|h^{\xi}\left(u^{*}, v^{*}, s^{*}\right)\right\|_{\infty},\left\|g^{\xi}\left(u^{*}, v^{*}, s^{*}\right)_{+}\right\|_{\infty},\left\|\kappa^{\xi}\left(u^{*}, v^{*}, s^{*}\right)_{+}\right\|_{\infty}\right\} \leq 10^{-4},
$$

i.e. quando a norma infinito da violação da inviabilidade do problema de viabilidade (4.1) não é maior que $10^{-4}$. Por outro lado, dado que $f^{\xi}(u, v, s)$ é a norma Euclidiana ao quadrado da violação da inviabilidade, experimentos numéricos irão mostrar que, na maioria dos casos, o critério de parada (4.6) implica

$$
f^{\xi}\left(u^{*}, v^{*}, s^{*}\right) \approx 10^{-16}
$$

e

$$
\max \left\{\left\|h^{\xi}\left(u^{*}, v^{*}, s^{*}\right)\right\|_{\infty},\left\|g^{\xi}\left(u^{*}, v^{*}, s^{*}\right)_{+}\right\|_{\infty},\left\|\kappa^{\xi}\left(u^{*}, v^{*}, s^{*}\right)_{+}\right\|_{\infty}\right\} \approx 10^{-8} .
$$

Valores de (4.8) e (4.9) serão reportados nos experimentos numéricos. Derivadas primeiras e a matriz Hessiana esparsa foram programadas manualmente. Mais ainda, a estratégia descrita em [BS08] para reduzir a complexidade na avaliação da função objetivo e das derivadas foi utilizada. Os códigos estão disponíveis em http://www.ime.usp.br/ egbirgin/packing/.

Como esperado, a estratégia multistart levou a resultados pobres. Quando utilizamos os pontos iniciais dos tipos R1, R2, R3 e R4, o Algoritmo 4.1 foi capaz de melhorar o número de itens fornecido pelo limite inferior dado pelo reticulado em poucos problemas, sendo a versão com R4 melhor ou igual do que as outras três em todas as instâncias. Mais ainda, como sugerido no final da Seção 4.1, o Algoritmo 4.1 com o tipo de pontos iniciais aleatórios R4 foi capaz de melhorar soluções para instâncias com um número pequeno de círculos (instâncias pequenas do conjunto 1) ou instâncias para as quais o limite inferior fornecido pelo reticulado é ruim (maioria das instâncias estreitas do conjunto 3). As Tabelas 4.2, 4.3, 4.4 e 4.5 mostram os resultados do Algoritmo 4.1 combinado com os pontos iniciais do tipo R1, R2, R3 e R4, respectivamente. Nas tabelas, as três primeiras colunas identificam as instâncias, incluindo seus limites inferiores computados. A coluna $m$ mostra o número de círculos empacotados. As colunas $f^{\xi}$ e $\left\|c^{\xi}\right\|_{\infty}$ correspondem a quantidades em (4.8) e (4.9), respectivamente, e quantificam a precisão das soluções computadas. Na coluna \#PI (número de pontos iniciais), A(B) reportam o número de pontos iniciais utilizados para empacotar $m_{l b}+1, \ldots, m$ círculos, significando que os Passos 3 e 4 do Algoritmo 4.1 foram executados A vezes e que dessas A rodadas B foram utilizadas para empacotar exatamente $m$ círculos. A coluna nomeada como "Tempo de CPU" mostra o tempo em segundos decorrido a partir do início até que a solução com $m$ círculos foi encontrada. A última coluna, \#PIA (número de pontos iniciais adicionais), mostra o número de tentativas sem sucesso para empacotar $m+1$ círculos que foram utilizadas no tempo de CPU que faltava para completar as 6 horas. A comparação entre o valor de \#PIA e o valor entre parêntesis na coluna \#PI serve de indicador para tentar definir se um item a mais não foi colocado porque de fato não cabe ou porque não houve tempo para tentar um número suficiente de vezes. As Figuras 4.5 e 4.6 mostram a representação gráfica das soluções obtidas com 
o uso do R4 e que melhoraram a solução dada pela estratégia do reticulado para o limite inferior. A representação gráfica sugere que algumas das soluções que melhoraram o limite inferior parecem estar longe de serem soluções ótimas.

\begin{tabular}{|c|c|c|c|c|c|c|c|c|c|}
\hline \multicolumn{3}{|c|}{ Instância } & \multicolumn{6}{|c|}{ Solução e esforço computacional } & Esforço extra \\
\hline & Nome & $m_{l b}$ & $m$ & $f^{\xi}$ & $\left\|c^{\xi}\right\|_{\infty}$ & & & Tempo de CPU (s.) & \#PIA \\
\hline \multirow{14}{*}{ 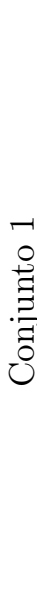 } & s1i01 & 4 & 5 & $6.1 \mathrm{D}-18$ & $3.3 \mathrm{D}-09$ & 15 & (15) & 0.02 & 18585732 \\
\hline & s1i02 & 12 & 13 & $2.6 \mathrm{D}-18$ & $1.4 \mathrm{D}-09$ & 361 & $(361)$ & 1.18 & 4331523 \\
\hline & s1i03 & 20 & 24 & $5.4 \mathrm{D}-16$ & $2.5 \mathrm{D}-08$ & 2824 & $(1437)$ & 47.74 & 729154 \\
\hline & s1i04 & 36 & 38 & $1.1 \mathrm{D}-16$ & $8.2 \mathrm{D}-09$ & 16030 & (13635) & 1597.49 & 156359 \\
\hline & s1i05 & 51 & 54 & $1.5 \mathrm{D}-17$ & $4.2 \mathrm{D}-09$ & 59372 & $(49125)$ & 9635.75 & 66665 \\
\hline & s1i06 & 74 & - & - & - & - & - & - & 55866 \\
\hline & sli07 & 95 & - & - & - & - & - & - & 30654 \\
\hline & s1i08 & 126 & - & - & - & - & - & - & 11775 \\
\hline & sli09 & 159 & - & - & - & - & - & - & 5711 \\
\hline & s1i10 & 192 & - & - & - & - & - & - & 4004 \\
\hline & sli11 & 238 & - & - & - & - & - & - & 1413 \\
\hline & sli12 & 273 & - & - & - & - & - & - & 1371 \\
\hline & sli13 & 320 & - & - & - & - & - & - & 819 \\
\hline & s1i14 & 374 & - & - & - & - & - & - & 528 \\
\hline \multirow{12}{*}{ 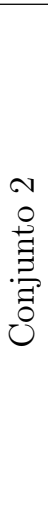 } & s2i01 & 369 & - & - & - & - & - & - & 545 \\
\hline & s2i02 & 367 & - & - & - & - & - & - & 491 \\
\hline & s2i03 & 363 & - & - & - & - & - & - & 543 \\
\hline & s2i04 & 364 & - & - & - & - & - & - & 456 \\
\hline & s2i05 & 361 & - & - & - & - & - & - & 418 \\
\hline & s2i06 & 354 & - & - & - & - & - & - & 462 \\
\hline & s2i07 & 346 & - & - & - & - & - & - & 710 \\
\hline & s2i08 & 341 & - & - & - & - & - & - & 873 \\
\hline & s2i09 & 331 & - & - & - & - & - & - & 1174 \\
\hline & s2i10 & 323 & - & - & - & - & - & - & 1341 \\
\hline & s2i11 & 296 & - & - & - & - & - & - & 2878 \\
\hline & s2i12 & 265 & - & - & - & - & - & - & 2879 \\
\hline \multirow{6}{*}{ 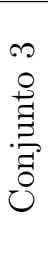 } & s3i01 & 87 & 88 & $1.4 \mathrm{D}-16$ & $7.8 \mathrm{D}-09$ & 23610 & (23610) & 4073.75 & 93439 \\
\hline & s3i02 & 56 & 60 & $7.4 \mathrm{D}-15$ & $1.1 \mathrm{D}-07$ & 11342 & $(7421)$ & 965.06 & 187600 \\
\hline & s3i03 & 107 & - & - & - & - & - & - & 50247 \\
\hline & s3i04 & 49 & 58 & $1.2 \mathrm{D}-09$ & $2.6 \mathrm{D}-05$ & 138954 & $(26622)$ & 11078.55 & 94913 \\
\hline & s3i05 & 128 & - & - & - & - & - & - & 29730 \\
\hline & s3i06 & 59 & 61 & $9.2 \mathrm{D}-17$ & $6.1 \mathrm{D}-09$ & 44671 & (11961) & 3606.36 & 187479 \\
\hline
\end{tabular}

Tabela 4.2: Resultados numéricos do Algoritmo 4.1 utilizando pontos iniciais do tipo R1. 


\begin{tabular}{|c|c|c|c|c|c|c|c|c|c|}
\hline \multicolumn{3}{|c|}{ Instância } & \multicolumn{6}{|c|}{ Solução e esforço computacional } & \multirow{2}{*}{$\frac{\text { Esforço extra }}{\text { \#PIA }}$} \\
\hline & Nome & $m_{l b}$ & $m$ & $f^{\xi}$ & $\left\|c^{\xi}\right\|_{\infty}$ & & PI & Tempo de CPU (s.) & \\
\hline & s1i01 & 4 & 5 & $3.0 \mathrm{D}-17$ & $6.7 \mathrm{D}-09$ & 23 & (23) & 0.02 & \\
\hline \multirow{13}{*}{$\begin{array}{l} \\
\\
\end{array}$} & sli02 & 12 & 13 & 8.3D-17 & $1.1 \mathrm{D}-08$ & 10513476 & (10513476) & 13805.59 & 294100 \\
\hline & sli03 & 20 & - & - & - & - & - & - & 5094742 \\
\hline & sli04 & 36 & - & - & - & - & - & - & 370295 \\
\hline & s1i05 & 51 & - & - & - & - & - & - & 212190 \\
\hline & s1i06 & 74 & - & - & - & - & - & - & 60002 \\
\hline & sli07 & 95 & - & - & - & - & - & - & 28812 \\
\hline & sli08 & 126 & - & - & - & - & - & - & 9715 \\
\hline & sli09 & 159 & - & - & - & - & - & - & 4428 \\
\hline & s1i10 & 192 & - & - & - & - & - & - & 2577 \\
\hline & sli11 & 238 & - & - & - & - & - & - & 1332 \\
\hline & sli12 & 273 & - & - & - & - & - & - & 1027 \\
\hline & sli13 & 320 & - & - & - & - & - & - & 578 \\
\hline & s1i14 & 374 & - & - & - & - & - & - & 364 \\
\hline \multirow{12}{*}{ 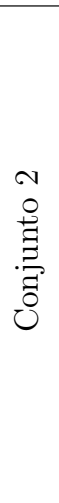 } & s2i01 & 369 & - & - & - & - & - & - & 498 \\
\hline & s2i02 & 367 & - & - & - & - & - & - & 537 \\
\hline & s2i03 & 363 & - & - & - & - & - & - & 531 \\
\hline & s2i04 & 364 & - & - & - & - & - & - & 490 \\
\hline & s2i05 & 361 & - & - & - & - & - & - & 399 \\
\hline & s2i06 & 354 & - & - & - & - & - & - & 542 \\
\hline & s2i07 & 346 & - & - & - & - & - & - & 578 \\
\hline & s2i08 & 341 & - & - & - & - & - & - & 995 \\
\hline & s2i09 & 331 & - & - & - & - & - & - & 2144 \\
\hline & s2i10 & 323 & - & - & - & - & - & - & 1528 \\
\hline & s2i11 & 296 & - & - & - & - & - & - & 21159 \\
\hline & s2i12 & 265 & - & - & - & - & - & - & 17960 \\
\hline \multirow{6}{*}{ 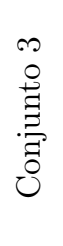 } & s3i01 & 87 & - & - & - & - & - & - & 32420 \\
\hline & s3i02 & 56 & - & - & - & - & - & - & 295331 \\
\hline & s3i03 & 107 & - & - & - & - & - & - & 150688 \\
\hline & s3i04 & 49 & - & - & - & - & - & - & 209600 \\
\hline & s3i05 & 128 & - & - & - & - & - & - & 108928 \\
\hline & s3i06 & 59 & - & - & - & - & - & - & 748470 \\
\hline
\end{tabular}

Tabela 4.3: Resultados numéricos do Algoritmo 4.1 utilizando pontos iniciais do tipo R2. 


\begin{tabular}{|c|c|c|c|c|c|c|c|c|c|}
\hline \multicolumn{3}{|c|}{ Instância } & \multicolumn{6}{|c|}{ Solução e esforço computacional } & Esforço extra \\
\hline & Nome & $m_{l b}$ & $m$ & $f^{\xi}$ & $\left\|c^{\xi}\right\|_{\infty}$ & & PI & Tempo de CPU (s.) & \#PIA \\
\hline \multirow{14}{*}{ 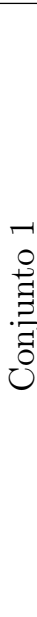 } & s1i01 & 4 & 5 & $2.1 \mathrm{D}-17$ & $4.3 \mathrm{D}-09$ & 8 & (8) & 0.01 & 18819733 \\
\hline & sli02 & 12 & 13 & $9.1 \mathrm{D}-18$ & $3.0 \mathrm{D}-09$ & 141 & (141) & 0.59 & 3215472 \\
\hline & s1i03 & 20 & 24 & $9.4 \mathrm{D}-17$ & $8.8 \mathrm{D}-09$ & 144 & $(92)$ & 3.41 & 498809 \\
\hline & s1i04 & 36 & 39 & $1.3 \mathrm{D}-15$ & $4.6 \mathrm{D}-08$ & 7249 & $(6814)$ & 1142.91 & 109375 \\
\hline & s1i05 & 51 & 56 & $2.3 \mathrm{D}-16$ & $1.2 \mathrm{D}-08$ & 19515 & (6091) & 4287.77 & 48055 \\
\hline & s1i06 & 74 & - & - & - & - & - & - & 43820 \\
\hline & s1i07 & 95 & - & - & - & - & - & - & 23946 \\
\hline & s1i08 & 126 & - & - & - & - & - & - & 8993 \\
\hline & s1i09 & 159 & - & - & - & - & - & - & 4529 \\
\hline & sli10 & 192 & - & - & - & - & - & - & 2909 \\
\hline & sli11 & 238 & - & - & - & - & - & - & 1095 \\
\hline & sli12 & 273 & - & - & - & - & - & - & 1072 \\
\hline & sli13 & 320 & - & - & - & - & - & - & 613 \\
\hline & sli14 & 374 & - & - & - & - & - & - & 392 \\
\hline \multirow{12}{*}{ 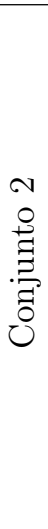 } & s2i01 & 369 & - & - & - & - & - & - & 435 \\
\hline & s2i02 & 367 & - & - & - & - & - & - & 478 \\
\hline & s2i03 & 363 & - & - & - & - & - & - & 466 \\
\hline & s2i04 & 364 & - & - & - & - & - & - & 354 \\
\hline & s2i05 & 361 & - & - & - & - & - & - & 308 \\
\hline & s2i06 & 354 & - & - & - & - & - & - & 421 \\
\hline & s2i07 & 346 & - & - & - & - & - & - & 416 \\
\hline & s2i08 & 341 & - & - & - & - & - & - & 643 \\
\hline & s2i09 & 331 & - & - & - & - & - & - & 712 \\
\hline & s2i10 & 323 & - & - & - & - & - & - & 1383 \\
\hline & s2i11 & 296 & - & - & - & - & - & - & 2177 \\
\hline & s2i12 & 265 & - & - & - & - & - & - & 2003 \\
\hline \multirow{6}{*}{ 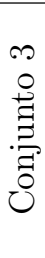 } & s3i01 & 87 & - & - & - & - & - & - & 79586 \\
\hline & s3i02 & 56 & 59 & $1.6 \mathrm{D}-17$ & $4.6 \mathrm{D}-09$ & 56980 & (20282) & 3894.28 & 215200 \\
\hline & s3i03 & 107 & - & - & - & - & - & - & 38627 \\
\hline & s3i04 & 49 & 58 & $3.3 \mathrm{D}-15$ & $4.4 \mathrm{D}-08$ & 204916 & (24962) & 18287.08 & 25572 \\
\hline & s3i05 & 128 & - & - & - & - & - & - & 23348 \\
\hline & s3i06 & 59 & 61 & $7.0 \mathrm{D}-17$ & $6.2 \mathrm{D}-09$ & 39577 & (36401) & 4344.92 & 142736 \\
\hline
\end{tabular}

Tabela 4.4: Resultados numéricos do Algoritmo 4.1 utilizando pontos iniciais do tipo R3. 


\begin{tabular}{|c|c|c|c|c|c|c|c|c|c|}
\hline \multicolumn{3}{|c|}{ Instância } & \multicolumn{6}{|c|}{ Solução e esforço computacional } & Esforço extra \\
\hline & Nome & $m_{l b}$ & $m$ & $f^{\xi}$ & $\left\|c^{\xi}\right\|_{\infty}$ & & PI & Tempo de CPU (s.) & \#PIA \\
\hline \multirow{14}{*}{ 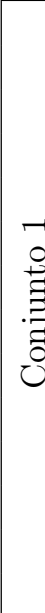 } & sli01 & 4 & 5 & $6.2 \mathrm{D}-18$ & $2.1 \mathrm{D}-09$ & 5 & (5) & 0.00 & 18382854 \\
\hline & s1i02 & 12 & 13 & $1.7 \mathrm{D}-16$ & $1.5 \mathrm{D}-08$ & 33 & (33) & 0.14 & 3083185 \\
\hline & s1i03 & 20 & 24 & $7.7 \mathrm{D}-17$ & $9.6 \mathrm{D}-09$ & 202 & $(26)$ & 4.13 & 652782 \\
\hline & s1i04 & 36 & 39 & $1.3 \mathrm{D}-16$ & $1.0 \mathrm{D}-08$ & 36082 & $(35485)$ & 4935.44 & 100794 \\
\hline & s1i05 & 51 & 56 & $2.4 \mathrm{D}-17$ & $5.2 \mathrm{D}-09$ & 13280 & $(4796)$ & 3248.33 & 55003 \\
\hline & s1i06 & 74 & 76 & $7.7 \mathrm{D}-16$ & $2.0 \mathrm{D}-08$ & 31888 & $(24132)$ & 20415.82 & 1522 \\
\hline & s1i07 & 95 & 97 & $7.6 \mathrm{D}-17$ & $7.1 \mathrm{D}-09$ & 13407 & $(8410)$ & 18193.36 & 2086 \\
\hline & s1i08 & 126 & - & - & - & - & - & - & 6364 \\
\hline & s1i09 & 159 & - & - & — & - & - & - & 3038 \\
\hline & s1i10 & 192 & - & - & - & - & - & - & 2074 \\
\hline & sli11 & 238 & - & - & - & - & - & - & 573 \\
\hline & sli12 & 273 & - & - & - & - & - & - & 669 \\
\hline & s1i13 & 320 & - & - & - & - & - & - & 364 \\
\hline & sli14 & 374 & - & - & - & - & - & - & 198 \\
\hline \multirow{12}{*}{ 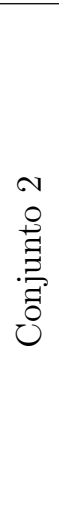 } & s2i01 & 369 & - & - & - & - & - & - & 178 \\
\hline & s2i02 & 367 & - & - & - & - & - & - & 313 \\
\hline & s2i03 & 363 & - & - & - & - & - & - & 269 \\
\hline & s2i04 & 364 & - & - & - & - & - & - & 269 \\
\hline & s2i05 & 361 & - & - & - & - & - & - & 224 \\
\hline & s2i06 & 354 & - & - & - & - & - & - & 229 \\
\hline & s2i07 & 346 & - & - & - & - & - & - & 387 \\
\hline & s2i08 & 341 & - & - & - & - & - & - & 605 \\
\hline & s2i09 & 331 & - & - & - & - & - & - & 802 \\
\hline & s2i10 & 323 & - & - & - & - & - & - & 1533 \\
\hline & s2i11 & 296 & - & - & - & - & - & - & 2117 \\
\hline & s2i12 & 265 & - & - & - & - & - & - & 1913 \\
\hline \multirow{6}{*}{ 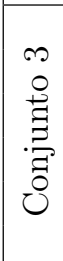 } & s3i01 & 87 & 98 & $2.9 \mathrm{D}-11$ & $4.1 \mathrm{D}-06$ & 34507 & $(1455)$ & 19705.80 & 260 \\
\hline & s3i02 & 56 & 60 & $5.2 \mathrm{D}-16$ & $8.6 \mathrm{D}-09$ & 1641 & (1610) & 245.50 & 12563 \\
\hline & s3i03 & 107 & 109 & $3.2 \mathrm{D}-16$ & $8.4 \mathrm{D}-09$ & 18872 & (9436) & 12838.60 & 1217 \\
\hline & s3i04 & 49 & 68 & $3.6 \mathrm{D}-16$ & $1.8 \mathrm{D}-08$ & 19419 & $(6254)$ & 5888.90 & 4109 \\
\hline & s3i05 & 128 & - & - & - & - & - & - & 2374 \\
\hline & s3i06 & 59 & 71 & $1.3 \mathrm{D}-16$ & $1.0 \mathrm{D}-08$ & 59180 & (33678) & 14992.50 & 2295 \\
\hline
\end{tabular}

Tabela 4.5: Resultados numéricos do Algoritmo 4.1 utilizando pontos iniciais do tipo R4. 


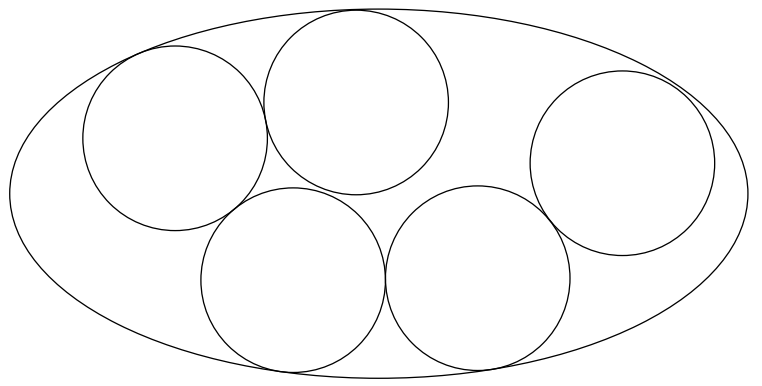

(a) s1i01

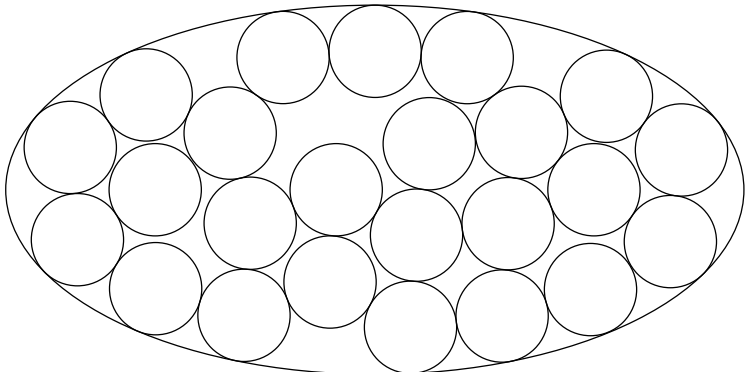

(c) $\mathrm{s} 1 \mathrm{i} 03$

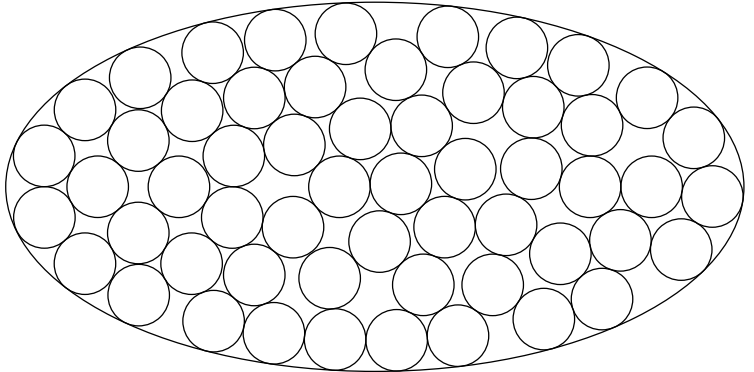

(e) $\mathrm{s} 1 \mathrm{i} 05$

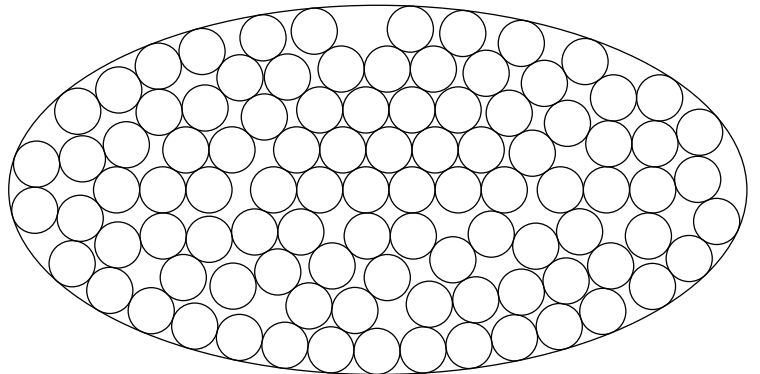

(g) s1i07

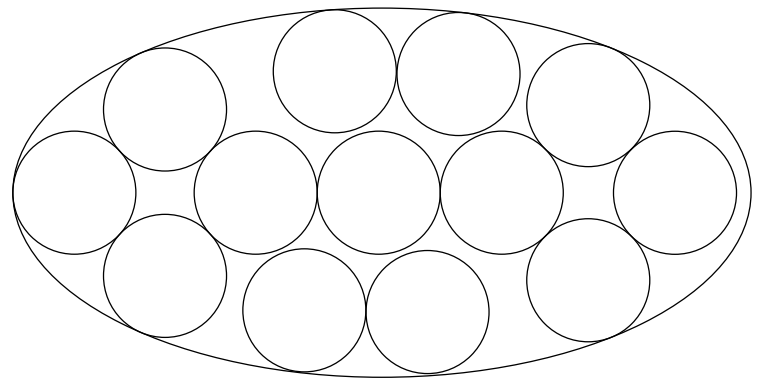

(b) s1i02

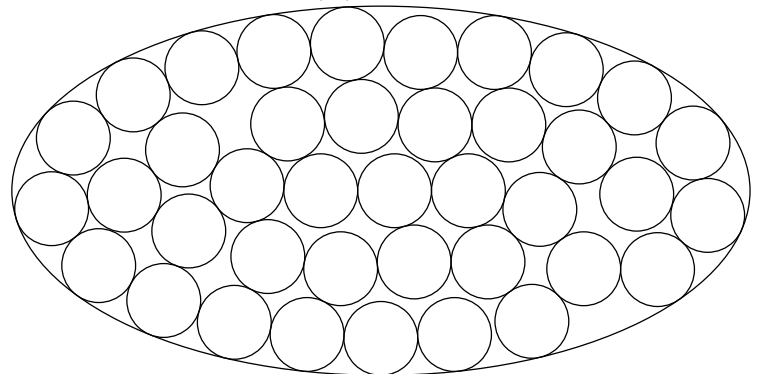

(d) $\mathrm{s} 1 \mathrm{i04}$

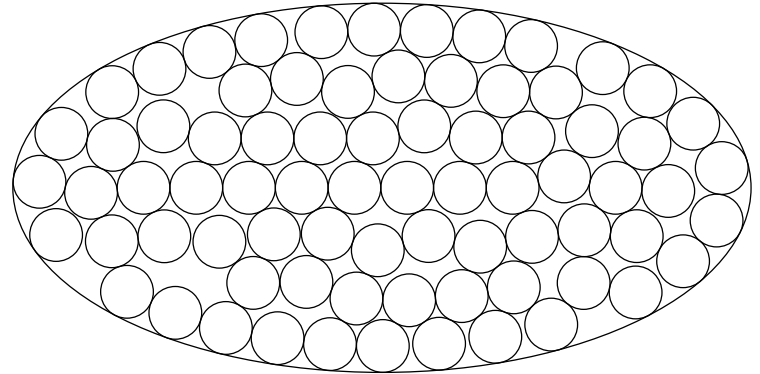

(f) s1i06 simples para instâncias do conjunto 1 que melhoraram a solução dada pelo limite inferior. 
(a) s3i01

(b) s3i02

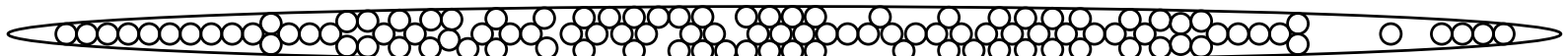

(c) s3i03

\section{0}

(d) $\mathrm{s} 3 \mathrm{i} 04$

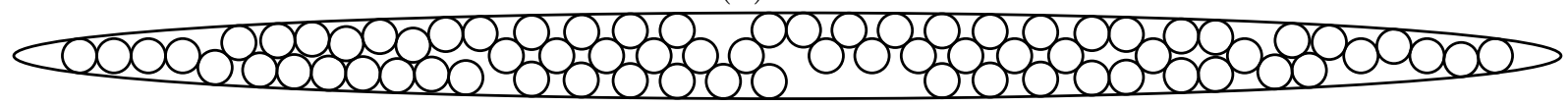

(e) $\mathrm{s} 3 \mathrm{i} 06$

Figura 4.6: Representação gráfica das soluções obtidas pelo Algoritmo $4.1 \mathrm{com}$ a versão do multistart simples para instâncias do conjunto 3 que melhoraram a solução dada pelo limite inferior.

\section{$4.3 \quad$ Tunneling}

Como o objetivo de aumentar as chances de encontrar minimizadores globais, uma estratégia de Tunneling [LM85, Sri06, BGR96, NG09] foi desenvolvida. Muitos pontos estacionários obtidos pelo Algoritmo 4.1 com os pontos iniciais do tipo R1, R2, R3 e R4 apresentam itens com seu centro na borda do contêiner (veja alguns exemplos na Figura 4.7). Consideramos então um movimento de tunneling que, quando aplicado a um ponto estacionário, tenta realocar o item que tem a maior contribuição na função objetivo (4.4) numa posição onde o valor de (4.4) seja estritamente menor.
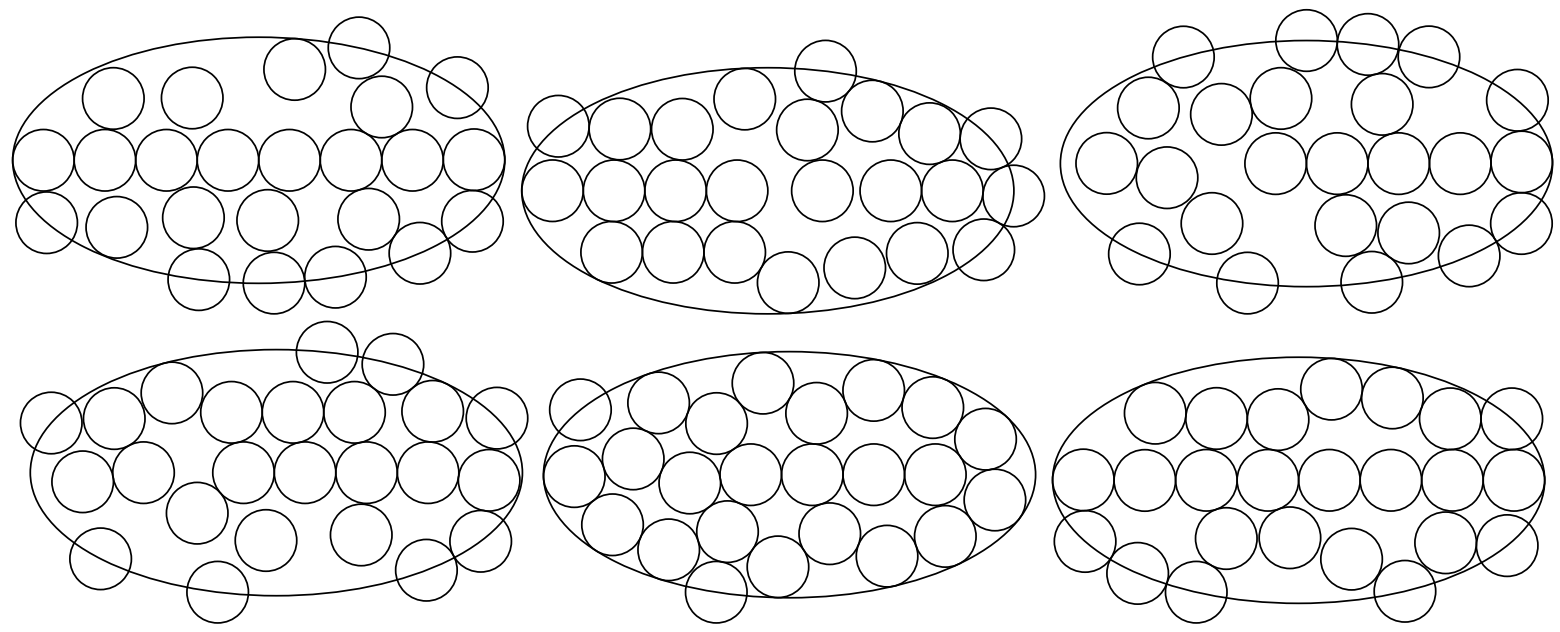

Figura 4.7: Exemplos de pontos estacionários obtidos na tentativa de encontrar uma solução com 24 itens com $r=1$ na elipse com $a=8$ e $b=4$.

A estratégia de empacotamento que utiliza o procedimento de tunneling pode ser descrita pelo Algoritmo 4.2. Todos os passos do Algoritmo 4.2 são idênticos aos do Algoritmo 4.1 com exceção dos Passos 5 e 6. São feitas $m$ tentativas aleatórias de movimentar o item que mais contribui com a função objetivo (4.4) para uma posição no interior da elipse onde a função (4.4) tenha um valor menor. Analisando a função objetivo em (4.4) é possível observar que um item $i$ determinado por 
$\left(u_{i}, v_{i}, s_{i}\right)$ com $\left(u_{i} / a\right)^{2}+\left(v_{i} / b\right)^{2}=1$ e $s_{i}=1$ (quer dizer, com seu centro na borda da elipse) contribui com valor 0.5 no somatório em (4.4). A mesma contribuição acontece se dois itens $i$ e $j$ são tais $\left(u_{i}, v_{i}, s_{i}\right)=\left(u_{j}, v_{j}, s_{j}\right)$, quer dizer, se os itens $i$ e $j$ estiverem totalmente sobrepostos. É por este motivo que realocar um item com o seu centro na borda da elipse para um outro lugar qualquer no interior da elipse tem probabilidade alta de produzir uma redução no valor da função objetivo.

Algoritmo 4.2 Sejam $a \geq b>0$ e $r>0$ constantes dadas.

Passo 1. Calcule o limite inferior $m_{l b}$, i.e. um valor $m_{l b} \geq 0$ tal que $\left(u^{*}, v^{*}, s^{*}\right) \in \mathbb{R}^{3 m_{l b}}$ com $f^{\xi}\left(u^{*}, v^{*}, s^{*}\right)=0$ seja conhecido (note que $m_{l b}=0$ é uma escolha válida). Faça $m_{+} \leftarrow 1 \mathrm{e}$ $m \leftarrow m_{l b}+m_{+}$.

Passo 2. Se o limite de tempo é atingido, pare declarando que $m^{*}=m-1$ itens foram empacotados.

Passo 3. Calcule um ponto inicial aleatório $\left(u_{\text {ini }}, v_{\text {ini }}, s_{\text {ini }}\right)$ para o problema (4.3).

Passo 4. Encontre um ponto estacionário $\left(u^{*}, v^{*}, s^{*}\right)$ de (4.3) iniciando a partir de $\left(u_{\text {ini }}, v_{\text {ini }}, s_{\text {ini }}\right)$.

Passo 5. Se $f^{\xi}\left(u^{*}, v^{*}, s^{*}\right)$ se anula, faça $m_{+} \leftarrow m_{+}+1, m \leftarrow m_{l b}+m_{+}$e vá para o Passo 2 .

Passo 6. Tente, com $m$ movimentos de tunneling a partir do ponto estacionário $\left(u^{*}, v^{*}, s^{*}\right)$, achar um novo ponto $\left(u^{\text {ini }}, v^{\text {ini }}, s^{\text {ini }}\right)$ tal que $f^{\xi}\left(u^{\text {ini }}, v^{\text {ini }}, s^{\text {ini }}\right)<f^{\xi}\left(u^{*}, v^{*}, s^{*}\right)$ (no caso, o melhor em $m$ tentativas). Em caso de sucesso, vá para o Passo 4. Caso contrário, vá para o Passo 2.

A Figura 4.8 representa uma sequência de minimizações feitas pelo Algoritmo 4.2 (a partir de pontos iniciais aleatórios do tipo R4) na tentativa de encontrar uma solução para o problema de empacotar 24 itens com $r=1$ na elipse com $a=8$ e $b=4$. Na figura, é possível observar que apenas dois pontos iniciais aleatórios foram necessários. O primeiro ponto inicial não levou a um minimizador global já que, após nove movimentos de tunneling bem sucedidos, a estratégia de tunneling não foi capaz de melhorar o valor da função objetivo do décimo ponto estacionário. A sequência de pontos estacionários e pontos iniciais gerados é ilustrada nas Figuras 4.9-4.10. Após o fracasso da estratégia de tunneling, um novo ponto inicial aleatório é gerado e, depois de cinco movimentos de tunneling bem sucedidos, a solução global é encontrada. A Figura 4.11 representa a sequência que leva a um minimizador global. Veja na Figura 4.10 que o ponto estacionário que não pode ser melhorado na primeira sequência de tunnelings tem valor de função objetivo da ordem de $10^{-6}$ e que não foi considerado uma solução global do problema por não satisfazer o critério (4.7).

As Tabelas 4.6, 4.7, 4.8 e 4.9 mostram os resultados do Algoritmo 4.2 utilizando os tipos de pontos iniciais R1, R2, R3 e R4, respectivamente. O Algoritmo 4.2 não conseguiu melhorar o limitante inferior dado pela estratégia do reticulado em apenas um problema do conjunto 1 utilizando o ponto inicial do tipo R4. Como nas tabelas anteriores, as três primeiras colunas identificam as instâncias, incluindo o seus limites inferiores computados. Nas tabelas, \#CA identifica o número de minimizações locais ou, equivalentemente, chamadas a Algencan. Nessa coluna, A(B) signifca que foram feitas, ao todo, A minimizações locais para empacotar $1,2, \ldots, m$ itens e que, dessas A minimizações, B foram utilizadas para achar o empacotamento com exatamente $m$ itens. Dessa forma, \#CA é comparável ao número de pontos iniciais utilizado pelo Algoritmo 4.1 que, no caso do Algoritmo 4.1 coincide com o número de minimizações locais. 


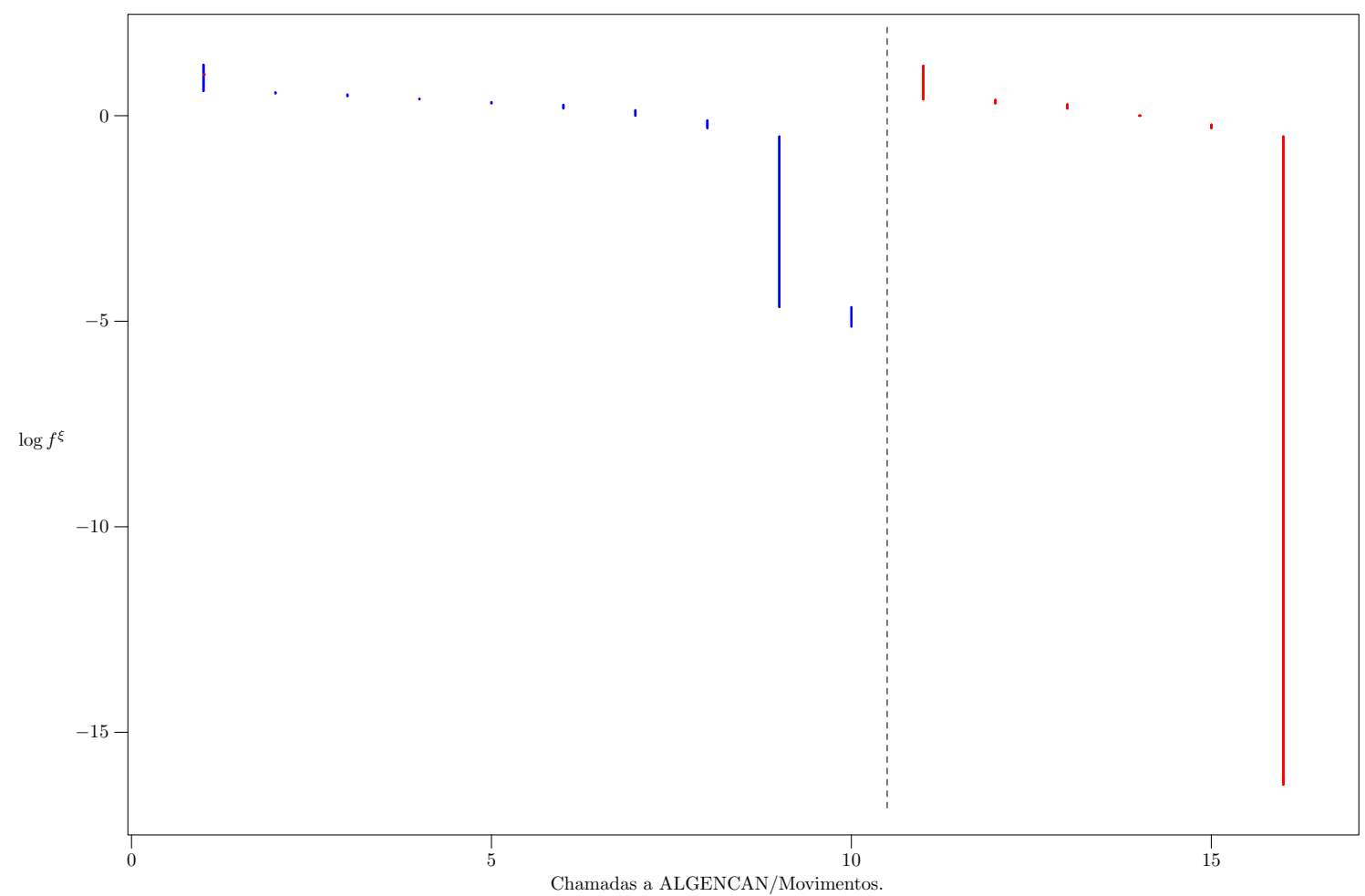

Figura 4.8: Sequência de minimizações feitas pelo Algoritmo 4.2 (a partir de pontos iniciai aleatórios do tipo R4) na tentativa de encontrar uma solução para o problema de empacotar 24 itens com $r=1$ na elipse com $a=8$ e $b=4$. A figura mostra que foram necessários dois pontos iniciais aleatórios, dezesseis minimizações locais, quatorze movimentos de tunneling. 


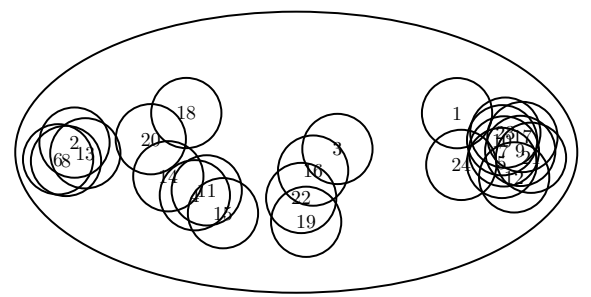

(a) $f^{\xi}=17.386469483344637$

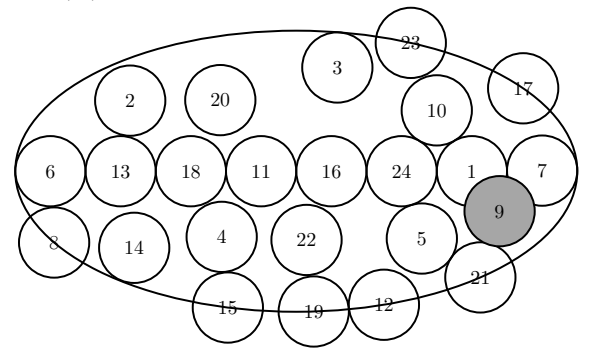

(c) $f^{\xi}=3.6811480347566103$

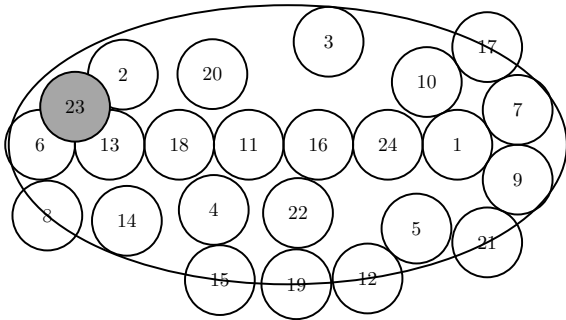

(e) $f^{\xi}=3.2583157037952484$

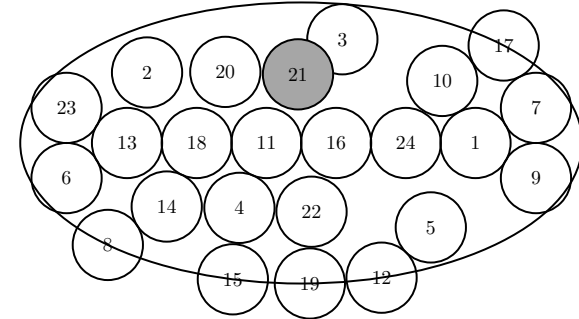

(g) $f^{\xi}=2.5653094375517895$

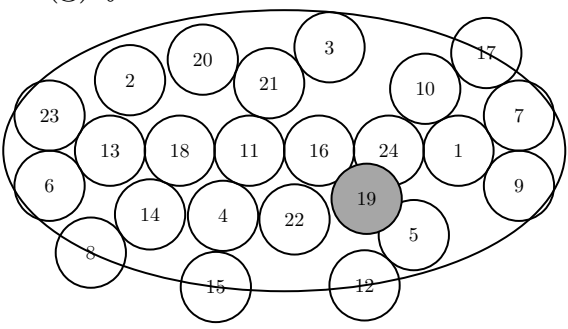

(i) $f^{\xi}=2.1391115345493437$

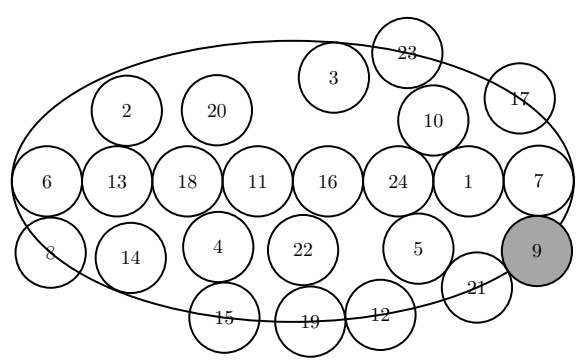

(b) $f^{\xi}=4.0000000000723599$

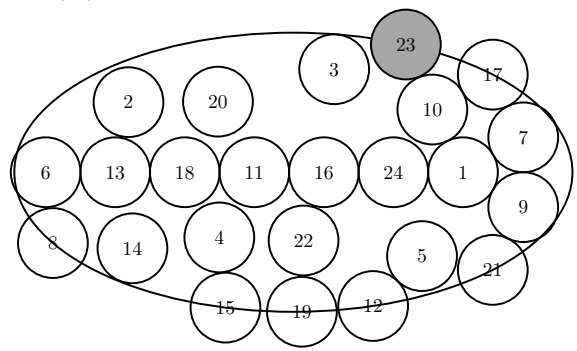

(d) $f^{\xi}=3.5006445177230123$

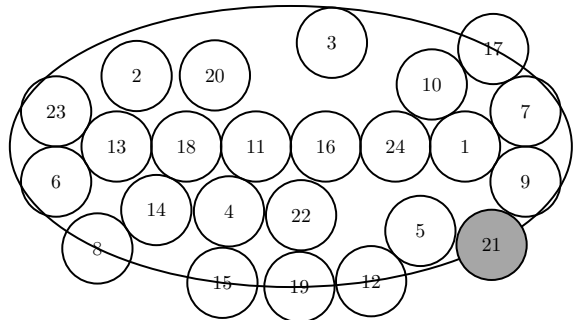

(f) $f^{\xi}=3.0032490001883527$

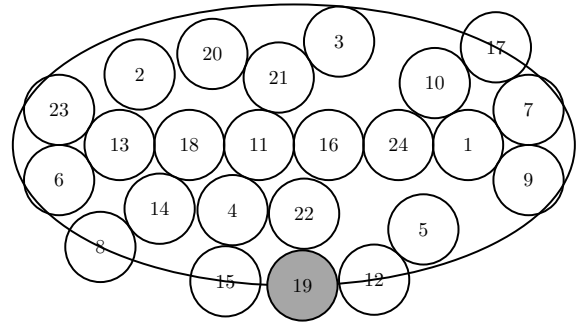

(h) $f^{\xi}=2.5032490001883527$

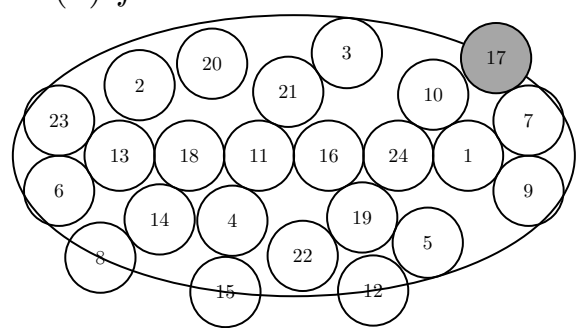

(j) $f^{\xi}=2.0032490001883527$

Figura 4.9: O gráfico em (a) mostra um ponto aleatório inicial do tipo R4 para o problema de empacotar 24 itens com $r=1$ na elipse com $a=8$ e $b=4$. O gráfico em (b) mostra o ponto estacionário encontrado partindo do ponto em (a). Em (c) é ilustrado o novo ponto inicial construído a partir de (b) utilizando a estratégia de tunneling. De (b) para (c), o item cinza foi realocado. De forma geral, os desenhos da direita mostram pontos estacionários e o desenho logo abaixo a esquerda representa um ponto inicial calculado usando um movimento de tunneling a partir de um ponto estacionário. 

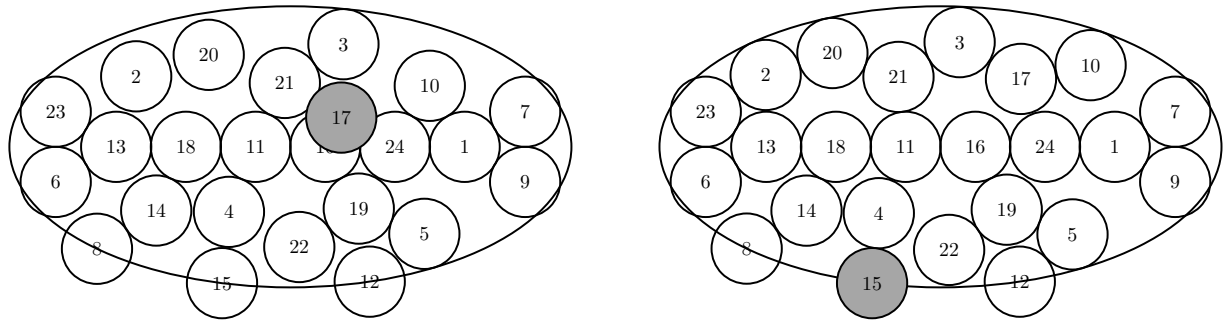

(k) $f^{\xi}=1.8392842545379235$

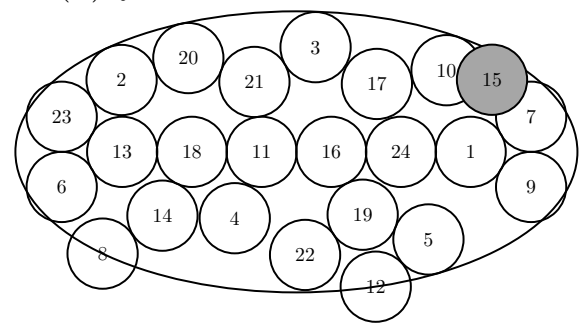

(l) $f^{\xi}=1.5032490001883523$

(m) $f^{\xi}=1.3562183025188674$
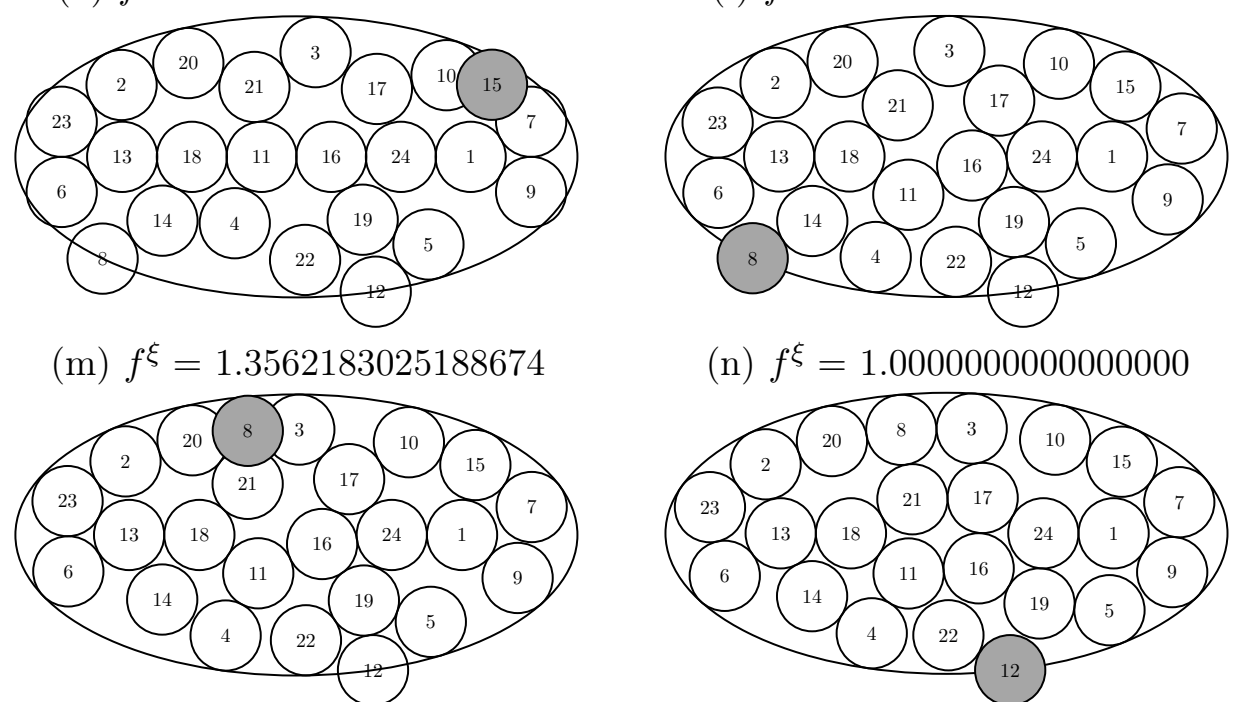

(n) $f^{\xi}=1.0000000000000000$

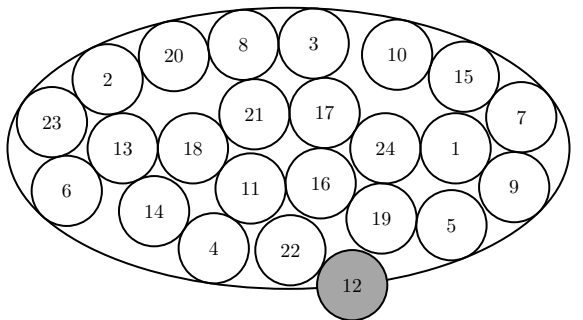

(o) $f^{\xi}=0.76704644257735977$

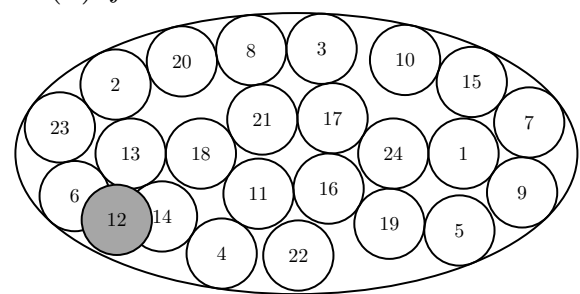

(p) $f^{\xi}=0.50000000000000000$

(q) $f^{\xi}=0.31136573868840467$
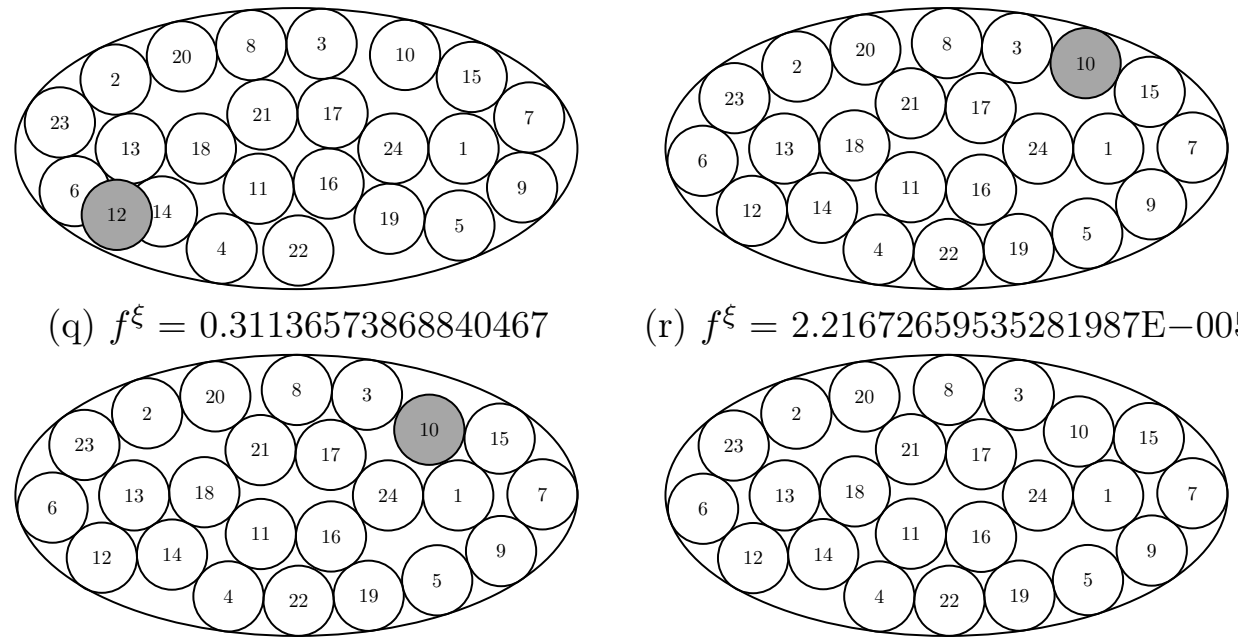

(r) $f^{\xi}=2.21672659535281987 \mathrm{E}-005$

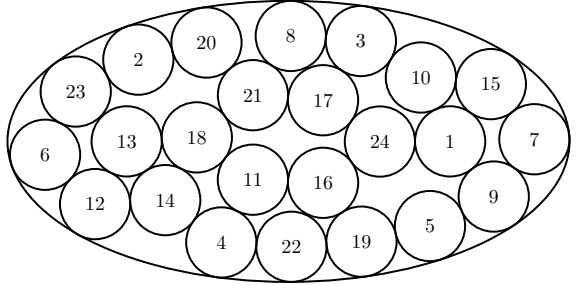

(s) $f^{\xi}=2.21130050036201885 \mathrm{E}-005$

(t) $f^{\xi}=7.36224937566463421 \mathrm{E}-006$

Figura 4.10: Continuação da Figura 4.9. A sequência termina no ponto estacionário ilustrado em (t) a partir do qual o movimento de tunneling não foi capaz de melhorar o valor da função objetivo. 


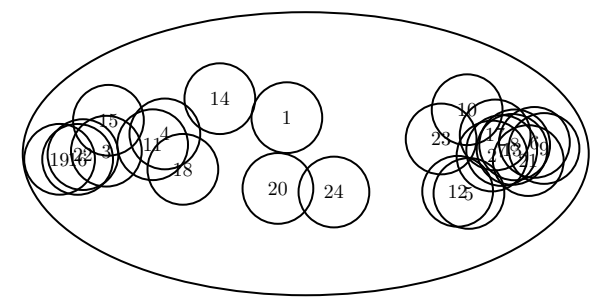

(a) $f^{\xi}=16.323046835981479$

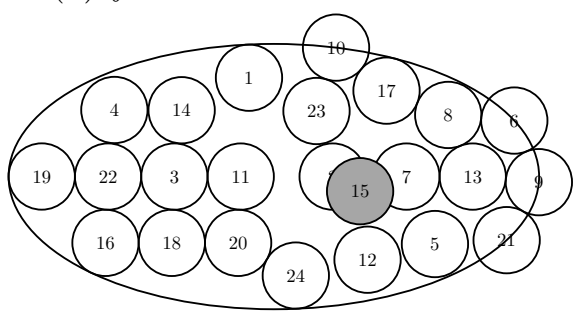

(c) $f^{\xi}=2.4119038429659976$

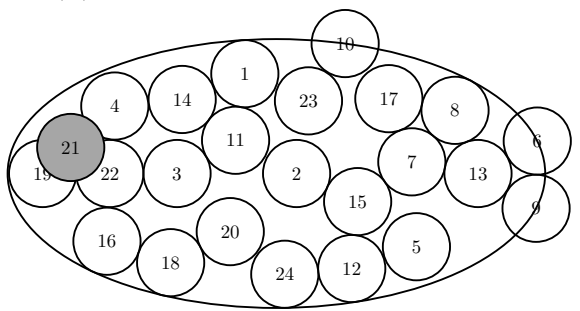

(e) $f^{\xi}=1.8765543263721911$

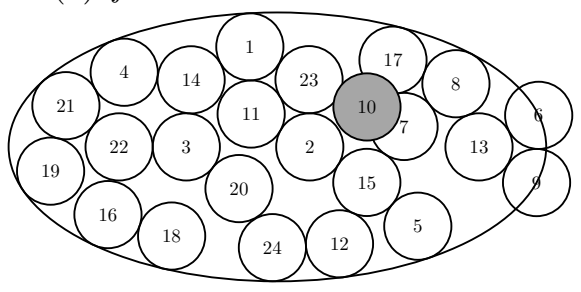

(g) $f^{\xi}=1.2578327777297733$

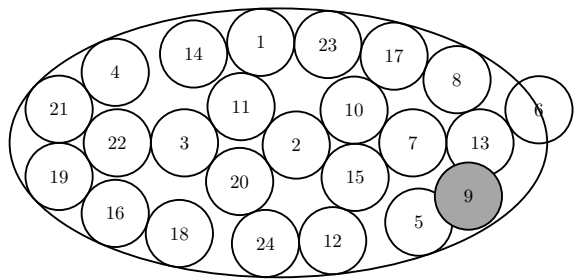

(i) $f^{\xi}=0.60495506610935346$

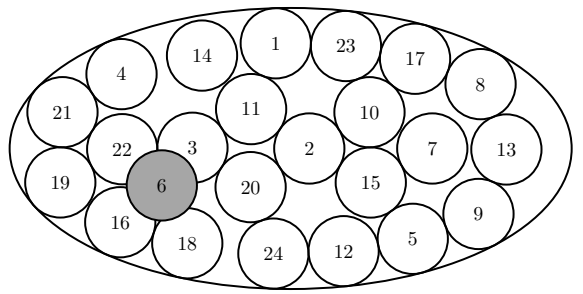

(k) $f^{\xi}=0.31180706748813836$

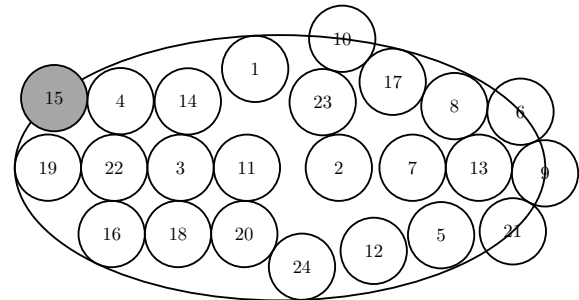

(b) $f^{\xi}=2.5000000000000000$

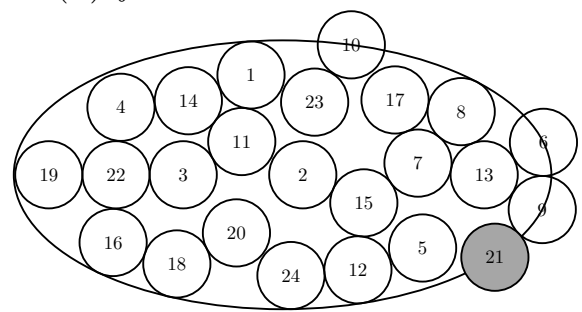

(d) $f^{\xi}=2.0000000000000000$

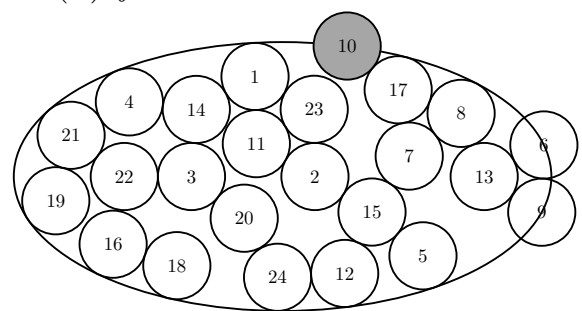

(f) $f^{\xi}=1.5000000000000000$

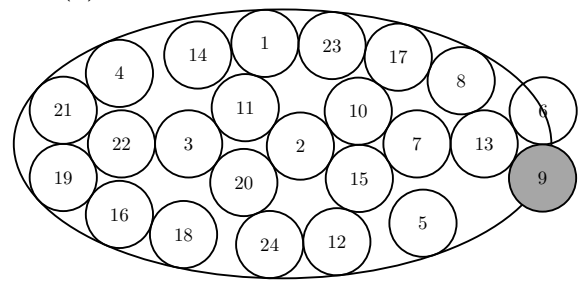

(h) $f^{\xi}=1.0000000000000007$

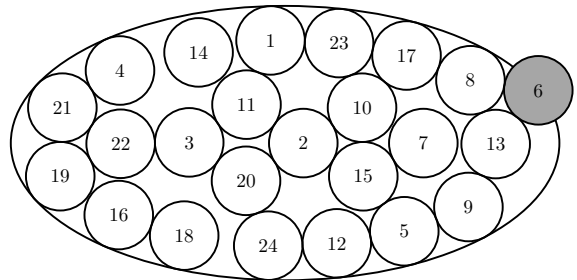

(j) $f^{\xi}=0.50000000000000033$

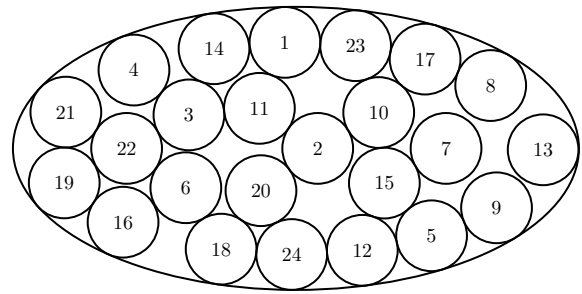

(l) $f^{\xi}=5.37193346381372110 \mathrm{E}-017$

Figura 4.11: Os gráficos mostram uma sequência de movimentos de tunneling que culmina na obtenção de um minimizador global para o problema de empacotar 24 itens com $r=1$ na elipse com $a=8$ e $b=4$. 


\begin{tabular}{|c|c|c|c|c|c|c|c|c|c|c|c|c|}
\hline \multicolumn{3}{|c|}{ Instância } & \multicolumn{8}{|c|}{ Solução e esforço computacional } & \multicolumn{2}{|c|}{ Esforço extra } \\
\hline & Nome & $m_{l b}$ & $m$ & $f^{\xi}$ & $\left\|c^{\xi}\right\|_{\infty}$ & & PI & & $\mathrm{CA}$ & Tempo de CPU (s.) & \#PIA & $\# \mathrm{CAA}$ \\
\hline \multirow{14}{*}{ 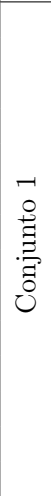 } & s1i01 & 4 & 5 & $8.0 \mathrm{D}-18$ & $3.1 \mathrm{D}-09$ & 8 & (8) & 14 & $(14)$ & 0.02 & 12306891 & 26995467 \\
\hline & s1i02 & 12 & 13 & $1.7 \mathrm{D}-16$ & $1.5 \mathrm{D}-08$ & 1 & (1) & 7 & $(7)$ & 0.03 & 989592 & 5798959 \\
\hline & s1i03 & 20 & 24 & $7.6 \mathrm{D}-17$ & $8.4 \mathrm{D}-09$ & 6 & $(2)$ & 66 & $(21)$ & 0.55 & 119838 & 1138007 \\
\hline & s1i04 & 36 & 39 & $6.5 \mathrm{D}-17$ & $5.5 \mathrm{D}-09$ & 11 & $(9)$ & 130 & (114) & 7.72 & 23030 & 287659 \\
\hline & s1i05 & 51 & 57 & $8.6 \mathrm{D}-11$ & $6.1 \mathrm{D}-06$ & 46 & (41) & 571 & $(518)$ & 117.69 & 6869 & 99224 \\
\hline & s1i06 & 74 & 78 & $1.7 \mathrm{D}-16$ & $1.3 \mathrm{D}-08$ & 8 & $(5)$ & 92 & $(67)$ & 42.48 & 2777 & 46573 \\
\hline & $\mathrm{s} 1 \mathrm{i} 07$ & 95 & 103 & $4.1 \mathrm{D}-16$ & $2.1 \mathrm{D}-08$ & 40 & (29) & 691 & (518) & 544.58 & 1173 & 22531 \\
\hline & s1i08 & 126 & 132 & $1.0 \mathrm{D}-08$ & $5.2 \mathrm{D}-05$ & 500 & $(486)$ & 10741 & (10444) & 17708.01 & 101 & 2251 \\
\hline & s1i09 & 159 & 164 & $3.5 \mathrm{D}-16$ & $1.4 \mathrm{D}-08$ & 12 & (8) & 259 & (174) & 765.64 & 264 & 6341 \\
\hline & s1i10 & 192 & 198 & $1.1 \mathrm{D}-16$ & $1.1 \mathrm{D}-08$ & 38 & (12) & 957 & $(320)$ & 3522.46 & 162 & 4080 \\
\hline & sli11 & 238 & 239 & $4.8 \mathrm{D}-16$ & $2.0 \mathrm{D}-08$ & 3 & $(3)$ & 104 & (104) & 677.66 & 88 & 2664 \\
\hline & s1i12 & 273 & 280 & $5.1 \mathrm{D}-15$ & $5.0 \mathrm{D}-08$ & 31 & (16) & 826 & $(442)$ & 9185.79 & 35 & 1093 \\
\hline & sli13 & 320 & 327 & $1.5 \mathrm{D}-16$ & $6.7 \mathrm{D}-09$ & 8 & (1) & 254 & $(36)$ & 2821.60 & 37 & 1240 \\
\hline & s1i14 & 374 & 378 & $1.2 \mathrm{D}-16$ & $1.2 \mathrm{D}-08$ & 6 & (1) & 227 & (43) & 3699.49 & 21 & 655 \\
\hline \multirow{12}{*}{ 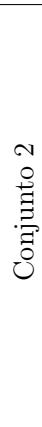 } & $\mathrm{s} 2 \mathrm{i} 01$ & 369 & 375 & $3.7 \mathrm{D}-16$ & $1.8 \mathrm{D}-08$ & 7 & $(2)$ & 234 & (74) & 4476.39 & 20 & 731 \\
\hline & s2i02 & 367 & 373 & $8.7 \mathrm{D}-17$ & $8.6 \mathrm{D}-09$ & 25 & (18) & 1040 & $(740)$ & 20789.62 & 1 & 54 \\
\hline & $\mathrm{s} 2 \mathrm{i} 03$ & 363 & 375 & $9.1 \mathrm{D}-17$ & $8.9 \mathrm{D}-09$ & 22 & (10) & 914 & (431) & 20395.01 & 1 & 40 \\
\hline & $\mathrm{s} 2 \mathrm{i} 04$ & 364 & 374 & $3.4 \mathrm{D}-17$ & $7.1 \mathrm{D}-09$ & 18 & $(3)$ & 844 & (143) & 18186.06 & 3 & 127 \\
\hline & $\mathrm{s} 2 \mathrm{i} 05$ & 361 & 375 & $4.4 \mathrm{D}-16$ & $1.7 \mathrm{D}-08$ & 21 & (6) & 1230 & $(345)$ & 22278.32 & 0 & 0 \\
\hline & $\mathrm{s} 2 \mathrm{i} 06$ & 354 & 371 & $2.7 \mathrm{D}-16$ & $9.9 \mathrm{D}-09$ & 18 & (1) & 1000 & (63) & 12462.39 & 7 & 453 \\
\hline & $\mathrm{s} 2 \mathrm{i} 07$ & 346 & 371 & $1.5 \mathrm{D}-16$ & $7.1 \mathrm{D}-09$ & 30 & (2) & 2608 & (149) & 20446.49 & 1 & 106 \\
\hline & $\mathrm{s} 2 \mathrm{i} 08$ & 341 & 365 & $8.3 \mathrm{D}-16$ & $2.5 \mathrm{D}-08$ & 25 & (1) & 2727 & (115) & 11375.97 & 11 & 959 \\
\hline & s2i09 & 331 & 358 & $1.8 \mathrm{D}-15$ & $5.4 \mathrm{D}-08$ & 34 & (4) & 3958 & $(407)$ & 17008.71 & 5 & 547 \\
\hline & $\mathrm{s} 2 \mathrm{i} 10$ & 323 & 358 & $6.3 \mathrm{D}-16$ & $1.7 \mathrm{D}-08$ & 40 & (1) & 4016 & (118) & 12642.86 & 12 & 1628 \\
\hline & s2i11 & 296 & 339 & $1.1 \mathrm{D}-15$ & $2.6 \mathrm{D}-08$ & 53 & (2) & 6778 & $(320)$ & 18643.27 & 5 & 752 \\
\hline & s2i12 & 265 & 325 & $2.3 \mathrm{D}-16$ & $9.7 \mathrm{D}-09$ & 77 & (2) & 8465 & $(233)$ & 18365.35 & 5 & 621 \\
\hline \multirow{6}{*}{ 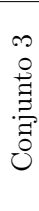 } & $\mathrm{s} 3 \mathrm{i} 01$ & 87 & 124 & $1.0 \mathrm{D}-12$ & $1.4 \mathrm{D}-06$ & 1087 & $(48)$ & 28826 & (1336) & 19349.43 & 121 & 3393 \\
\hline & s3i02 & 56 & 60 & $2.4 \mathrm{D}-16$ & $6.0 \mathrm{D}-09$ & 334 & $(330)$ & 2739 & (2695) & 205.84 & 33612 & 286025 \\
\hline & s3i03 & 107 & 140 & $1.3 \mathrm{D}-15$ & $4.2 \mathrm{D}-08$ & 602 & $(159)$ & 27893 & $(7501)$ & 16276.74 & 181 & 8624 \\
\hline & s3i04 & 49 & 75 & $1.2 \mathrm{D}-16$ & $6.5 \mathrm{D}-09$ & 7116 & $(5843)$ & 126897 & $(105276)$ & 17926.95 & 1471 & 27004 \\
\hline & $\mathrm{s} 3 \mathrm{i} 05$ & 128 & 150 & $8.2 \mathrm{D}-17$ & $7.8 \mathrm{D}-09$ & 135 & $(111)$ & 8019 & (6821) & 4670.34 & 395 & 24159 \\
\hline & s3i06 & 59 & 78 & $9.5 \mathrm{D}-17$ & $6.4 \mathrm{D}-09$ & 2339 & (1458) & 57384 & $(36221)$ & 8527.52 & 3266 & 82840 \\
\hline
\end{tabular}

Tabela 4.6: Resultados numéricos do Algoritimo 4.2 utilizando pontos iniciais do tipo R1.

É importante ressaltar que uma variedade grande de movimentos de tunneling foi idealizada e testada numericamente. Todas elas levaram a resultados práticos muito similares aos da estratégia aqui descrita.

\subsection{Alternativas ao multistart}

As estratégias descritas nas seções anteriores para gerar os pontos iniciais aleatórios no procedimento de empacotamento com $\bar{m}$ itens não faz o uso da solução conhecida para empacotar $\bar{m}-1$. Esta decisão tem uma motivação clara: ser capaz de rapidamente reproduzir um dado resultado (solução) sem ter que reproduzir todo o experimento desde o início. Entretanto, dado o fraco desempenho de tal estratégia, descrevemos na presente seção estratégias adicionais para gerar pontos iniciais que fazem uso das soluções previamente computadas. As três estratégias consideradas são

M1: O ponto inicial para empacotar $\bar{m}$ círculos sempre será a solução conhecida para empacotar $\bar{m}-1$ círculos mais um valor inicial gerado aleatoriamente pela estratégia $\mathrm{R} 4$ para as três variáveis associadas ao círculo novo.

M2: A primeira tentativa para esta estratégia funciona como M1. Entretanto, quando um ponto estácionário indesejado para empacotar $\bar{m}$ círculos for obtido, novos valores aleatórios (como na estratégia R4) serão dados para o círculo de maior inviabilidade, preservando os outros 


\begin{tabular}{|c|c|c|c|c|c|c|c|c|c|c|c|c|}
\hline \multicolumn{3}{|c|}{ Instância } & \multicolumn{8}{|c|}{ Solução e esforço computacional } & \multicolumn{2}{|c|}{ Esforço extra } \\
\hline & Nome & $m_{l b}$ & $m$ & $f^{\xi}$ & $\left\|c^{\xi}\right\|_{\infty}$ & & PI & & $\mathrm{A}$ & Tempo de CPU (s.) & \#PIA & \#CAA \\
\hline \multirow{14}{*}{ 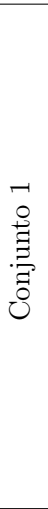 } & s1i01 & 4 & 5 & $7.8 \mathrm{D}-17$ & $1.2 \mathrm{D}-08$ & 1 & (1) & 2 & (2) & 0.00 & 9211152 & 28185044 \\
\hline & sli02 & 12 & 13 & $1.5 \mathrm{D}-16$ & $1.2 \mathrm{D}-08$ & 1 & (1) & 6 & (6) & 0.03 & 800063 & 7501041 \\
\hline & sli03 & 20 & 24 & $1.4 \mathrm{D}-17$ & $4.0 \mathrm{D}-09$ & 6 & (3) & 109 & $(57)$ & 0.91 & 109271 & 1951818 \\
\hline & sli04 & 36 & 39 & $3.3 \mathrm{D}-17$ & $4.0 \mathrm{D}-09$ & 5 & (3) & 126 & $(75)$ & 3.40 & 20429 & 475852 \\
\hline & sli05 & 51 & 57 & $4.6 \mathrm{D}-17$ & $6.2 \mathrm{D}-09$ & 21 & (14) & 548 & (363) & 50.97 & 6325 & 167201 \\
\hline & s1i06 & 74 & 78 & $1.3 \mathrm{D}-16$ & $9.5 \mathrm{D}-09$ & 12 & $(9)$ & 397 & $(296)$ & 86.53 & 2462 & 82509 \\
\hline & sli07 & 95 & 103 & $1.0 \mathrm{D}-16$ & $1.2 \mathrm{D}-08$ & 16 & (4) & 598 & $(154)$ & 214.07 & 1046 & 40295 \\
\hline & sli08 & 126 & 132 & $2.9 \mathrm{D}-16$ & $1.2 \mathrm{D}-08$ & 293 & (264) & 12351 & (11122) & 12625.30 & 193 & 8109 \\
\hline & sli09 & 159 & 164 & $1.0 \mathrm{D}-14$ & $5.8 \mathrm{D}-08$ & 20 & $(12)$ & 915 & (549) & 1535.34 & 223 & 10066 \\
\hline & sli10 & 192 & 198 & $6.1 \mathrm{D}-17$ & $5.2 \mathrm{D}-09$ & 33 & (13) & 1684 & (671) & 3926.56 & 130 & 6526 \\
\hline & sli11 & 238 & 239 & $1.1 \mathrm{D}-16$ & $1.2 \mathrm{D}-08$ & 6 & (6) & 339 & (339) & 1650.61 & 75 & 4115 \\
\hline & sli12 & 273 & 280 & $1.1 \mathrm{D}-16$ & $1.0 \mathrm{D}-08$ & 53 & (44) & 3221 & $(2680)$ & 20463.95 & 3 & 185 \\
\hline & sli13 & 320 & 327 & $1.2 \mathrm{D}-16$ & $9.1 \mathrm{D}-09$ & 7 & (1) & 450 & (69) & 3366.75 & 28 & 1771 \\
\hline & sli14 & 374 & 377 & $4.4 \mathrm{D}-16$ & $2.6 \mathrm{D}-08$ & 3 & (1) & 210 & $(71)$ & 2757.90 & 18 & 1285 \\
\hline \multirow{12}{*}{ 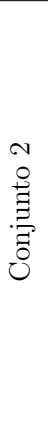 } & s2i01 & 369 & 376 & $6.9 \mathrm{D}-17$ & $6.3 \mathrm{D}-09$ & 18 & (5) & 1328 & $(372)$ & 16499.32 & 6 & 432 \\
\hline & s2i02 & 367 & 373 & $2.8 \mathrm{D}-16$ & $1.5 \mathrm{D}-08$ & 12 & (1) & 947 & $(78)$ & 9688.88 & 11 & 871 \\
\hline & s2i03 & 363 & 374 & $9.6 \mathrm{D}-17$ & $8.9 \mathrm{D}-09$ & 20 & (6) & 1623 & (481) & 17962.88 & 3 & 255 \\
\hline & s2i04 & 364 & 371 & $2.1 \mathrm{D}-16$ & $1.3 \mathrm{D}-08$ & 20 & (5) & 1784 & (438) & 21756.15 & 0 & 0 \\
\hline & s2i05 & 361 & 376 & $5.2 \mathrm{D}-16$ & $2.2 \mathrm{D}-08$ & 19 & (1) & 1736 & $(89)$ & 20697.79 & 1 & 86 \\
\hline & s2i06 & 354 & 370 & $3.3 \mathrm{D}-16$ & $9.5 \mathrm{D}-09$ & 23 & (1) & 2390 & (103) & 18416.55 & 2 & 209 \\
\hline & s2i07 & 346 & 371 & $3.4 \mathrm{D}-16$ & $2.0 \mathrm{D}-08$ & 28 & (2) & 3083 & (233) & 19886.63 & 2 & 217 \\
\hline & s2i08 & 341 & 365 & $1.1 \mathrm{D}-16$ & $9.0 \mathrm{D}-09$ & 28 & (2) & 3609 & $(237)$ & 16040.58 & 6 & 767 \\
\hline & s2i09 & 331 & 359 & $6.1 \mathrm{D}-16$ & $1.4 \mathrm{D}-08$ & 38 & (5) & 5175 & (711) & 20462.60 & 2 & 243 \\
\hline & s2i10 & 323 & 359 & $2.4 \mathrm{D}-15$ & $5.9 \mathrm{D}-08$ & 50 & (6) & 7987 & (997) & 21541.82 & 1 & 43 \\
\hline & s2i11 & 296 & 339 & $3.7 \mathrm{D}-16$ & $1.4 \mathrm{D}-08$ & 51 & (2) & 10623 & $(440)$ & 20115.89 & 3 & 614 \\
\hline & s2i12 & 265 & 322 & $2.9 \mathrm{D}-16$ & $1.2 \mathrm{D}-08$ & 65 & (1) & 11017 & (193) & 19598.44 & 3 & 539 \\
\hline \multirow{6}{*}{ 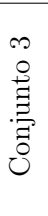 } & s3i01 & 87 & 122 & $1.4 \mathrm{D}-17$ & $2.7 \mathrm{D}-09$ & 712 & (329) & 30889 & $(14805)$ & 14961.49 & 273 & 12510 \\
\hline & s3i02 & 56 & 60 & $5.1 \mathrm{D}-17$ & $3.7 \mathrm{D}-09$ & 27 & $(24)$ & 391 & (355) & 19.23 & 28693 & 432262 \\
\hline & s3i03 & 107 & 139 & $3.5 \mathrm{D}-17$ & $3.7 \mathrm{D}-09$ & 144 & $(33)$ & 10036 & $(2433)$ & 4609.47 & 422 & 31291 \\
\hline & s3i04 & 49 & 74 & $2.9 \mathrm{D}-17$ & $5.4 \mathrm{D}-09$ & 2368 & (589) & 66744 & (17037) & 8880.07 & 2998 & 88739 \\
\hline & s3i05 & 128 & 149 & $7.0 \mathrm{D}-16$ & $1.7 \mathrm{D}-08$ & 58 & (33) & 5267 & (3096) & 2020.47 & 395 & 37314 \\
\hline & s3i06 & 59 & 78 & $3.3 \mathrm{D}-16$ & $1.9 \mathrm{D}-08$ & 3489 & (2319) & 136109 & (91099) & 16572.32 & 949 & 37741 \\
\hline
\end{tabular}

Tabela 4.7: Resultados numéricos do Algoritimo 4.2 utilizando pontos iniciais do tipo R2. 


\begin{tabular}{|c|c|c|c|c|c|c|c|c|c|c|c|c|}
\hline \multicolumn{3}{|c|}{ Instância } & \multicolumn{8}{|c|}{ Solução e esforço computacional } & \multicolumn{2}{|c|}{ Esforço extra } \\
\hline & Nome & $m_{l b}$ & $m$ & $f^{\xi}$ & $\left\|c^{\xi}\right\|_{\infty}$ & & PI & & $\mathrm{CA}$ & Tempo de CPU (s.) & \#PIA & \#CAA \\
\hline \multirow{14}{*}{ 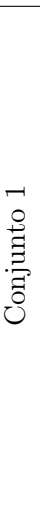 } & sli01 & 4 & 5 & $2.1 \mathrm{D}-17$ & $4.3 \mathrm{D}-09$ & 8 & (8) & 8 & (8) & 0.01 & 15585240 & 27032833 \\
\hline & sli02 & 12 & 13 & $4.9 \mathrm{D}-17$ & $9.3 \mathrm{D}-09$ & 3 & (3) & 17 & (17) & 0.04 & 1376861 & 5200118 \\
\hline & sli03 & 20 & 24 & $6.9 \mathrm{D}-17$ & $5.7 \mathrm{D}-09$ & 4 & (1) & 32 & $(8)$ & 0.36 & 140039 & 858151 \\
\hline & sli04 & 36 & 39 & $4.2 \mathrm{D}-15$ & $4.3 \mathrm{D}-08$ & 11 & (9) & 91 & (69) & 6.16 & 25780 & 233686 \\
\hline & s1i05 & 51 & 57 & $4.2 \mathrm{D}-17$ & $5.0 \mathrm{D}-09$ & 10 & (5) & 137 & (62) & 21.49 & 7298 & 82786 \\
\hline & s1i06 & 74 & 78 & $2.0 \mathrm{D}-16$ & $7.8 \mathrm{D}-09$ & 33 & (30) & 516 & (468) & 195.48 & 2880 & 39603 \\
\hline & sli07 & 95 & 103 & $5.8 \mathrm{D}-16$ & $2.1 \mathrm{D}-08$ & 52 & $(44)$ & 938 & $(771)$ & 750.31 & 1217 & 20168 \\
\hline & s1i08 & 126 & 132 & $9.5 \mathrm{D}-16$ & $1.6 \mathrm{D}-08$ & 161 & (145) & 3052 & $(2685)$ & 5737.44 & 421 & 7983 \\
\hline & sli09 & 159 & 164 & $3.3 \mathrm{D}-16$ & $1.7 \mathrm{D}-08$ & 14 & $(10)$ & 317 & $(230)$ & 833.50 & 274 & 5756 \\
\hline & sli10 & 192 & 199 & $7.9 \mathrm{D}-13$ & $7.0 \mathrm{D}-07$ & 162 & (145) & 3834 & (3418) & 18232.32 & 29 & 680 \\
\hline & sli11 & 238 & 239 & $1.2 \mathrm{D}-16$ & $1.1 \mathrm{D}-08$ & 4 & (4) & 107 & (107) & 843.75 & 93 & 2399 \\
\hline & s1i12 & 273 & 280 & $2.5 \mathrm{D}-16$ & $1.7 \mathrm{D}-08$ & 31 & (3) & 832 & 87 & 8163.66 & 41 & 1115 \\
\hline & sli13 & 320 & 327 & $1.9 \mathrm{D}-16$ & $1.0 \mathrm{D}-08$ & 13 & (4) & 381 & (124) & 5408.83 & 32 & 897 \\
\hline & sli14 & 374 & 377 & $2.8 \mathrm{D}-17$ & $4.2 \mathrm{D}-09$ & 21 & (16) & 647 & (496) & 15734.63 & 8 & 206 \\
\hline \multirow{12}{*}{ 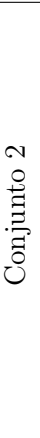 } & s2i01 & 369 & 376 & $6.1 \mathrm{D}-16$ & $1.9 \mathrm{D}-08$ & 16 & $(8)$ & 595 & (312) & 10536.75 & 13 & 485 \\
\hline & s2i02 & 367 & 373 & $3.2 \mathrm{D}-16$ & $1.5 \mathrm{D}-08$ & 18 & (9) & 872 & (437) & 14230.97 & 7 & 348 \\
\hline & s2i03 & 363 & 374 & $5.6 \mathrm{D}-16$ & $3.1 \mathrm{D}-08$ & 13 & (2) & 775 & (141) & 10028.47 & 11 & 688 \\
\hline & s2i04 & 364 & 371 & $2.8 \mathrm{D}-17$ & $3.0 \mathrm{D}-09$ & 10 & (3) & 842 & (264) & 10286.15 & 9 & 727 \\
\hline & s2i05 & 361 & 374 & $7.9 \mathrm{D}-17$ & $5.3 \mathrm{D}-09$ & 17 & (3) & 1488 & (271) & 17643.73 & 3 & 269 \\
\hline & s2i06 & 354 & 371 & $1.1 \mathrm{D}-16$ & $6.2 \mathrm{D}-09$ & 25 & (4) & 2445 & (396) & 23013.65 & 0 & 0 \\
\hline & s2i07 & 346 & 369 & $6.6 \mathrm{D}-16$ & $2.6 \mathrm{D}-08$ & 25 & (2) & 2739 & (221) & 16549.69 & 4 & 434 \\
\hline & s2i08 & 341 & 367 & $1.2 \mathrm{D}-15$ & $2.3 \mathrm{D}-08$ & 28 & (2) & 3661 & (266) & 14640.91 & 7 & 926 \\
\hline & s2i09 & 331 & 358 & $3.8 \mathrm{D}-15$ & $6.3 \mathrm{D}-08$ & 38 & (7) & 5417 & (974) & 20433.28 & 2 & 260 \\
\hline & s2i10 & 323 & 359 & $2.0 \mathrm{D}-16$ & $1.1 \mathrm{D}-08$ & 50 & (4) & 7598 & (653) & 20812.31 & 2 & 234 \\
\hline & s2i11 & 296 & 337 & $8.9 \mathrm{D}-15$ & $1.3 \mathrm{D}-07$ & 53 & (2) & 6986 & (305) & 19002.94 & 4 & 561 \\
\hline & s2i12 & 265 & 326 & $2.1 \mathrm{D}-15$ & $2.3 \mathrm{D}-08$ & 78 & (4) & 7294 & (501) & 15333.37 & 12 & 1490 \\
\hline \multirow{6}{*}{ 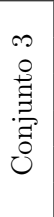 } & s3i01 & 87 & 120 & $6.4 \mathrm{D}-09$ & $6.1 \mathrm{D}-05$ & 683 & (308) & 20178 & $(9386)$ & 13345.90 & 37 & 1143 \\
\hline & s3i02 & 56 & 60 & $9.9 \mathrm{D}-16$ & $3.6 \mathrm{D}-08$ & 29 & $(26)$ & 303 & (268) & 19.22 & 19539 & 209064 \\
\hline & s3i03 & 107 & 140 & $1.5 \mathrm{D}-16$ & $7.0 \mathrm{D}-09$ & 458 & (259) & 22127 & (12569) & 11412.12 & 98 & 4775 \\
\hline & s3i04 & 49 & 73 & $2.8 \mathrm{D}-17$ & $4.5 \mathrm{D}-09$ & 1162 & (978) & 21736 & (18564) & 3305.39 & 3683 & 71842 \\
\hline & s3i05 & 128 & 149 & $1.5 \mathrm{D}-16$ & $4.8 \mathrm{D}-09$ & 59 & $(35)$ & 3312 & $(1956)$ & 1590.44 & 332 & 19182 \\
\hline & s3i06 & 59 & 77 & $5.4 \mathrm{D}-15$ & $9.7 \mathrm{D}-08$ & 341 & (154) & 8025 & (3771) & 1052.01 & 3751 & 91768 \\
\hline
\end{tabular}

Tabela 4.8: Resultados numéricos do Algoritimo 4.2 utilizando pontos iniciais do tipo R3. 


\begin{tabular}{|c|c|c|c|c|c|c|c|c|c|c|c|c|}
\hline \multicolumn{3}{|c|}{ Instância } & \multicolumn{8}{|c|}{ Solução e esforço computacional } & \multicolumn{2}{|c|}{ Esforço extra } \\
\hline & Nome & $m_{l b}$ & $m$ & $f^{\xi}$ & $\left\|c^{\xi}\right\|_{\infty}$ & & PI & & $\mathrm{CA}$ & Tempo de CPU (s.) & \#PIA & \#CAA \\
\hline \multirow{14}{*}{ 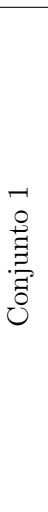 } & s1i01 & 4 & 5 & $6.2 \mathrm{D}-18$ & $2.1 \mathrm{D}-09$ & 5 & (5) & 6 & (6) & 0.00 & 19662697 & 27135292 \\
\hline & sli02 & 12 & 13 & $1.1 \mathrm{D}-16$ & 9.9D-09 & 3 & (3) & 13 & (13) & 0.04 & 1351672 & 5592068 \\
\hline & sli03 & 20 & 24 & $5.4 \mathrm{D}-17$ & $5.9 \mathrm{D}-09$ & 5 & (2) & 35 & (16) & 0.54 & 127878 & 1131284 \\
\hline & sli04 & 36 & 39 & $7.8 \mathrm{D}-17$ & $6.9 \mathrm{D}-09$ & 23 & $(21)$ & 350 & $(308)$ & 17.91 & 23229 & 310516 \\
\hline & sli05 & 51 & 57 & $5.2 \mathrm{D}-17$ & $5.3 \mathrm{D}-09$ & 37 & $(24)$ & 642 & $(401)$ & 93.05 & 6935 & 103167 \\
\hline & sli06 & 74 & 78 & $5.8 \mathrm{D}-17$ & $8.0 \mathrm{D}-09$ & 18 & $(15)$ & 342 & (237) & 103.15 & 2889 & 43759 \\
\hline & sli07 & 95 & 103 & $1.4 \mathrm{D}-15$ & $3.5 \mathrm{D}-08$ & 11 & $(2)$ & 221 & $(37)$ & 131.43 & 1255 & 18415 \\
\hline & sli08 & 126 & 132 & $9.5 \mathrm{D}-18$ & $3.1 \mathrm{D}-09$ & 503 & (489) & 7850 & $(7612)$ & 17490.30 & 113 & 1705 \\
\hline & sli09 & 159 & 164 & $5.9 \mathrm{D}-17$ & $5.0 \mathrm{D}-09$ & 15 & (10) & 211 & $(142)$ & 805.97 & 282 & 4588 \\
\hline & sli10 & 192 & 199 & $8.2 \mathrm{D}-16$ & $1.6 \mathrm{D}-08$ & 185 & (107) & 3403 & (1967) & 20075.47 & 14 & 220 \\
\hline & sli11 & 238 & - & - & - & - & - & - & - & - & 109 & 2050 \\
\hline & sli12 & 273 & 280 & $3.1 \mathrm{D}-09$ & $2.9 \mathrm{D}-05$ & 16 & (4) & 322 & $(82)$ & 4957.64 & 52 & 1015 \\
\hline & sli13 & 320 & 328 & $1.3 \mathrm{D}-16$ & $1.3 \mathrm{D}-08$ & 17 & (6) & 365 & (121) & 6616.77 & 31 & 592 \\
\hline & sli14 & 374 & 377 & $2.8 \mathrm{D}-15$ & $4.3 \mathrm{D}-08$ & 22 & (14) & 502 & (334) & 14953.91 & 9 & 174 \\
\hline \multirow{12}{*}{ 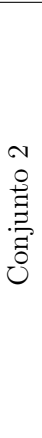 } & s2i01 & 369 & 375 & $8.6 \mathrm{D}-16$ & $2.6 \mathrm{D}-08$ & 9 & $(3)$ & 269 & $(87)$ & 5640.54 & 21 & 587 \\
\hline & $\mathrm{s} 2 \mathrm{i} 02$ & 367 & 373 & $8.5 \mathrm{D}-16$ & $1.8 \mathrm{D}-08$ & 10 & (1) & 400 & (33) & 7276.83 & 15 & 586 \\
\hline & s2i03 & 363 & 375 & $5.8 \mathrm{D}-16$ & $1.5 \mathrm{D}-08$ & 24 & (11) & 1139 & (533) & 19344.92 & 2 & 111 \\
\hline & s2i04 & 364 & 374 & $1.2 \mathrm{D}-16$ & $6.1 \mathrm{D}-09$ & 19 & (1) & 1161 & $(61)$ & 22261.46 & 0 & 0 \\
\hline & s2i05 & 361 & 374 & $1.3 \mathrm{D}-15$ & $3.4 \mathrm{D}-08$ & 15 & (2) & 1286 & (161) & 14737.87 & 5 & 357 \\
\hline & s2i06 & 354 & 370 & $7.2 \mathrm{D}-16$ & $2.1 \mathrm{D}-08$ & 17 & (1) & 1686 & (100) & 13095.99 & 7 & 645 \\
\hline & s2i07 & 346 & 370 & $1.9 \mathrm{D}-16$ & $9.6 \mathrm{D}-09$ & 28 & (2) & 3171 & $(215)$ & 20976.06 & 1 & 113 \\
\hline & s2i08 & 341 & 366 & $4.8 \mathrm{D}-16$ & $1.2 \mathrm{D}-08$ & 29 & (3) & 3818 & $(384)$ & 16641.86 & 5 & 614 \\
\hline & s2i09 & 331 & 359 & $2.8 \mathrm{D}-16$ & $1.4 \mathrm{D}-08$ & 33 & (1) & 4692 & (153) & 17276.01 & 5 & 664 \\
\hline & s2i10 & 323 & 359 & $2.5 \mathrm{D}-16$ & $1.1 \mathrm{D}-08$ & 45 & (7) & 6575 & (1143) & 18523.94 & 4 & 655 \\
\hline & s2i11 & 296 & 335 & $2.9 \mathrm{D}-16$ & $7.4 \mathrm{D}-09$ & 48 & (7) & 6118 & (1006) & 16498.49 & 9 & 1266 \\
\hline & s2i12 & 265 & 325 & $4.1 \mathrm{D}-15$ & $6.7 \mathrm{D}-08$ & 69 & (5) & 6958 & (693) & 14515.61 & 12 & 1520 \\
\hline \multirow{6}{*}{ 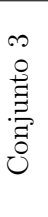 } & s3i01 & 87 & 123 & $1.2 \mathrm{D}-16$ & $1.4 \mathrm{D}-08$ & 1036 & $(435)$ & 24332 & (11003) & 14619.59 & 450 & 11553 \\
\hline & s3i02 & 56 & 60 & $3.3 \mathrm{D}-17$ & $2.7 \mathrm{D}-09$ & 29 & $(26)$ & 106 & (98) & 10.56 & 61155 & 249444 \\
\hline & s3i03 & 107 & 140 & $4.3 \mathrm{D}-17$ & $3.5 \mathrm{D}-09$ & 556 & (211) & 27154 & (10756) & 14981.67 & 223 & 11401 \\
\hline & s3i04 & 49 & 73 & $1.6 \mathrm{D}-14$ & $1.7 \mathrm{D}-07$ & 1508 & (378) & 21812 & $(5695)$ & 2846.76 & 9505 & 146682 \\
\hline & s3i05 & 128 & 150 & $5.0 \mathrm{D}-15$ & $6.5 \mathrm{D}-08$ & 422 & (378) & 26016 & (23497) & 16474.75 & 121 & 7458 \\
\hline & s3i06 & 59 & 77 & $3.2 \mathrm{D}-18$ & $1.5 \mathrm{D}-09$ & 224 & $(55)$ & 5298 & (1304) & 702.43 & 5982 & 146793 \\
\hline
\end{tabular}

Tabela 4.9: Resultados numéricos do Algoritimo 4.2 utilizando pontos iniciais do tipo R4. 


\begin{tabular}{|c|c|c|c|c|c|c|c|c|c|}
\hline \multicolumn{3}{|c|}{ Instância } & \multicolumn{6}{|c|}{ Solução e esforço computacional } & Esforço extra \\
\hline & Nome & $m_{l b}$ & $m$ & $f^{\xi}$ & $\left\|c^{\xi}\right\|_{\infty}$ & & & Tempo de CPU (s.) & \#PIA \\
\hline \multirow{14}{*}{ 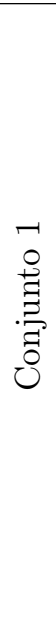 } & s1i01 & 4 & 5 & $2.7 \mathrm{D}-18$ & $2.3 \mathrm{D}-09$ & 6 & (6) & 0.00 & 23460130 \\
\hline & s1i02 & 12 & 13 & $1.0 \mathrm{D}-16$ & $9.8 \mathrm{D}-09$ & 720 & $(720)$ & 2.11 & 4659750 \\
\hline & s1i03 & 20 & 24 & $9.6 \mathrm{D}-17$ & $1.1 \mathrm{D}-08$ & 4 & (1) & 0.06 & 708338 \\
\hline & s1i04 & 36 & 39 & $2.9 \mathrm{D}-16$ & $1.1 \mathrm{D}-08$ & 158 & $(151)$ & 18.13 & 282306 \\
\hline & s1i05 & 51 & 57 & $1.6 \mathrm{D}-16$ & $1.1 \mathrm{D}-08$ & 301 & $(296)$ & 89.62 & 95656 \\
\hline & s1i06 & 74 & 78 & $1.2 \mathrm{D}-15$ & $2.2 \mathrm{D}-08$ & 209 & (198) & 162.08 & 37045 \\
\hline & s1i07 & 95 & 103 & $2.3 \mathrm{D}-12$ & $9.9 \mathrm{D}-07$ & 15 & (8) & 24.61 & 18221 \\
\hline & s1i08 & 126 & 132 & $9.6 \mathrm{D}-17$ & $6.7 \mathrm{D}-09$ & 87 & (5) & 274.92 & 9587 \\
\hline & s1i09 & 159 & 164 & $1.1 \mathrm{D}-17$ & $1.7 \mathrm{D}-09$ & 92 & (84) & 488.65 & 4862 \\
\hline & s1i10 & 192 & 198 & $3.8 \mathrm{D}-09$ & $3.1 \mathrm{D}-05$ & 135 & (128) & 808.40 & 2233 \\
\hline & s1i11 & 238 & 239 & $3.9 \mathrm{D}-16$ & $1.4 \mathrm{D}-08$ & 2 & (2) & 42.43 & 1351 \\
\hline & s1i12 & 273 & 281 & $2.2 \mathrm{D}-17$ & $2.5 \mathrm{D}-09$ & 77 & (70) & 2002.26 & 915 \\
\hline & s1i13 & 320 & 327 & $1.3 \mathrm{D}-16$ & $6.1 \mathrm{D}-09$ & 21 & (13) & 887.23 & 517 \\
\hline & s1i14 & 374 & 378 & $5.2 \mathrm{D}-17$ & $5.5 \mathrm{D}-09$ & 13 & $(6)$ & 960.37 & 307 \\
\hline \multirow{12}{*}{ 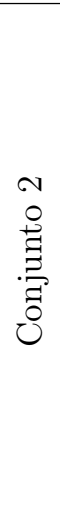 } & s2i01 & 369 & 376 & $2.0 \mathrm{D}-17$ & $3.6 \mathrm{D}-09$ & 20 & (14) & 1220.52 & 383 \\
\hline & s2i02 & 367 & 377 & $6.1 \mathrm{D}-17$ & $5.5 \mathrm{D}-09$ & 17 & (8) & 871.21 & 353 \\
\hline & s2i03 & 363 & 376 & $1.2 \mathrm{D}-16$ & $7.4 \mathrm{D}-09$ & 28 & (6) & 1735.57 & 341 \\
\hline & s2i04 & 364 & 378 & $3.2 \mathrm{D}-16$ & $1.3 \mathrm{D}-08$ & 339 & $(224)$ & 19106.20 & 53 \\
\hline & s2i05 & 361 & 375 & $2.1 \mathrm{D}-15$ & $4.3 \mathrm{D}-08$ & 80 & $(37)$ & 3717.94 & 422 \\
\hline & s2i06 & 354 & 371 & $6.9 \mathrm{D}-16$ & $2.2 \mathrm{D}-08$ & 550 & (234) & 18711.12 & 91 \\
\hline & s2i07 & 346 & 373 & $2.8 \mathrm{D}-15$ & $5.0 \mathrm{D}-08$ & 239 & (204) & 10490.40 & 313 \\
\hline & s2i08 & 341 & 366 & $2.9 \mathrm{D}-11$ & $4.8 \mathrm{D}-06$ & 189 & (59) & 6061.91 & 884 \\
\hline & s2i09 & 331 & 365 & $7.7 \mathrm{D}-17$ & $3.5 \mathrm{D}-09$ & 297 & $(22)$ & 6251.26 & 718 \\
\hline & s2i10 & 323 & 357 & $1.5 \mathrm{D}-15$ & $3.1 \mathrm{D}-08$ & 934 & (157) & 18528.08 & 199 \\
\hline & s2i11 & 296 & 353 & $5.1 \mathrm{D}-16$ & $2.0 \mathrm{D}-08$ & 540 & (136) & 7096.15 & 1112 \\
\hline & s2i12 & 265 & 324 & $1.8 \mathrm{D}-15$ & $3.9 \mathrm{D}-08$ & 2185 & $(242)$ & 20997.28 & 147 \\
\hline \multirow{6}{*}{ 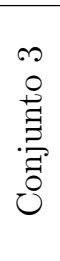 } & s3i01 & 87 & 118 & $1.4 \mathrm{D}-08$ & $6.4 \mathrm{D}-05$ & 18078 & (9972) & 10548.71 & 18630 \\
\hline & s3i02 & 56 & 57 & $2.0 \mathrm{D}-17$ & $6.0 \mathrm{D}-09$ & 5 & (5) & 0.13 & 605187 \\
\hline & s3i03 & 107 & 140 & $2.0 \mathrm{D}-14$ & $1.9 \mathrm{D}-07$ & 3136 & (2333) & 2414.27 & 25360 \\
\hline & s3i04 & 49 & 67 & $4.6 \mathrm{D}-16$ & $2.7 \mathrm{D}-08$ & 17826 & (9775) & 1919.16 & 169738 \\
\hline & s3i05 & 128 & 147 & $2.5 \mathrm{D}-16$ & $9.5 \mathrm{D}-09$ & 383 & $(150)$ & 449.84 & 21535 \\
\hline & s3i06 & 59 & 77 & $1.8 \mathrm{D}-16$ & $9.0 \mathrm{D}-09$ & 361 & $(37)$ & 61.32 & 129557 \\
\hline
\end{tabular}

Tabela 4.10: Desempenho do Algoritmo 4.1 associado à estratégia M1 para o cálculo dos pontos iniciais.

valores do ponto estacionário. Esse ponto estacionário modificado é utilizado como ponto inicial para a próxima tentativa.

M3: Esta estratégia funciona como M2, mas as variáveis de um círculo escolhido aleatoriamente são modificadas em vez das variáveis associadas ao disco mais inviável.

Note que a estratégia M2 assemelha-se à estratégia de tunneling, embora uma melhoria na função objetivo no novo ponto inicial com respeito ao ponto estacionário corrente não seja necessária. As tabelas 4.10-4.12 mostram os resultados do Algoritmo 4.1 associado às estratégias M1-M3 para o cálculo dos pontos iniciais. As Figuras 4.12-4.16 ilustram a melhor solução encontrada por uma das três estratégias. As tabelas mostram que as estratégias M1, M2 e M3 encontram as melhores soluções (entre as soluções encontradas pelas três estratégias) em 17, 27 e 15 instâncias (em um total de 32), respectivamente. As tabelas também mostram que, se a estratégia M3 for ignorada, todas as melhores soluções ainda serão encontradas pelas estratégias M1 e M2, e que, entre M1 e M2, nenhuma das duas é melhor que a outra. 


\begin{tabular}{|c|c|c|c|c|c|c|c|c|c|}
\hline \multicolumn{3}{|c|}{ Instância } & \multicolumn{6}{|c|}{ Solução e esforço computacional } & \multirow{2}{*}{$\frac{\text { Esforço extra }}{\# \text { PIA }}$} \\
\hline & Nome & $m_{l b}$ & $m$ & $f^{\xi}$ & $\left\|c^{\xi}\right\|_{\infty}$ & & PI & Tempo de CPU (s.) & \\
\hline \multirow{14}{*}{ 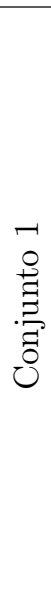 } & s1i01 & 4 & 5 & $4.1 \mathrm{D}-19$ & $7.8 \mathrm{D}-10$ & 9 & $(9)$ & 0.01 & 26068471 \\
\hline & $\mathrm{s} 1 \mathrm{i} 02$ & 12 & 13 & $1.5 \mathrm{D}-16$ & $1.5 \mathrm{D}-08$ & 2 & $(2)$ & 0.01 & 4774069 \\
\hline & s1i03 & 20 & 24 & $9.6 \mathrm{D}-17$ & $1.1 \mathrm{D}-08$ & 4 & (1) & 0.06 & 907210 \\
\hline & s1i04 & 36 & 39 & $2.7 \mathrm{D}-17$ & $3.8 \mathrm{D}-09$ & 49 & $(44)$ & 5.06 & 261714 \\
\hline & $\mathrm{s} 1 \mathrm{i} 05$ & 51 & 57 & $4.5 \mathrm{D}-17$ & $9.1 \mathrm{D}-09$ & 47 & $(42)$ & 12.29 & 95777 \\
\hline & s1i06 & 74 & 78 & $7.2 \mathrm{D}-17$ & $5.6 \mathrm{D}-09$ & 8 & $(3)$ & 10.23 & 37881 \\
\hline & s1i07 & 95 & 103 & $9.5 \mathrm{D}-17$ & $7.6 \mathrm{D}-09$ & 80 & $(73)$ & 89.06 & 28480 \\
\hline & s1i08 & 126 & 132 & $1.0 \mathrm{D}-15$ & $2.1 \mathrm{D}-08$ & 313 & $(298)$ & 762.19 & 10864 \\
\hline & s1i09 & 159 & 164 & $2.3 \mathrm{D}-16$ & $1.2 \mathrm{D}-08$ & 8 & (3) & 36.75 & 6807 \\
\hline & s1i10 & 192 & 199 & $1.8 \mathrm{D}-16$ & $1.5 \mathrm{D}-08$ & 44 & (1) & 359.77 & 4197 \\
\hline & s1i11 & 238 & 239 & $3.6 \mathrm{D}-16$ & $2.3 \mathrm{D}-08$ & 9 & (9) & 218.12 & 2823 \\
\hline & s1i12 & 273 & 280 & $2.8 \mathrm{D}-16$ & $1.7 \mathrm{D}-08$ & 7 & (1) & 98.31 & 2049 \\
\hline & s1i13 & 320 & 328 & $1.1 \mathrm{D}-09$ & $2.1 \mathrm{D}-05$ & 555 & $(543)$ & 11780.04 & 583 \\
\hline & s1i14 & 374 & 378 & $1.7 \mathrm{D}-15$ & $2.6 \mathrm{D}-08$ & 52 & (34) & 3140.71 & 645 \\
\hline \multirow{12}{*}{ 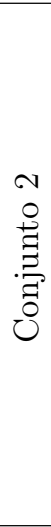 } & $\mathrm{s} 2 \mathrm{i} 01$ & 369 & 377 & $3.4 \mathrm{D}-16$ & $1.2 \mathrm{D}-08$ & 351 & $(342)$ & 12567.15 & 348 \\
\hline & $\mathrm{s} 2 \mathrm{i} 02$ & 367 & 376 & $3.4 \mathrm{D}-16$ & $2.1 \mathrm{D}-08$ & 9 & (1) & 160.42 & 1096 \\
\hline & $\mathrm{s} 2 \mathrm{i} 03$ & 363 & 376 & $7.6 \mathrm{D}-16$ & $1.9 \mathrm{D}-08$ & 324 & (291) & 12559.34 & 284 \\
\hline & s2i04 & 364 & 378 & $7.0 \mathrm{D}-16$ & $1.6 \mathrm{D}-08$ & 390 & (1) & 11094.14 & 386 \\
\hline & $\mathrm{s} 2 \mathrm{i} 05$ & 361 & 379 & $4.1 \mathrm{D}-16$ & $1.4 \mathrm{D}-08$ & 462 & $(250)$ & 15327.75 & 280 \\
\hline & $\mathrm{s} 2 \mathrm{i} 06$ & 354 & 378 & $3.8 \mathrm{D}-16$ & $1.2 \mathrm{D}-08$ & 278 & (1) & 8496.39 & 657 \\
\hline & $\mathrm{s} 2 \mathrm{i} 07$ & 346 & 375 & $1.4 \mathrm{D}-09$ & $2.0 \mathrm{D}-05$ & 830 & $(548)$ & 16983.64 & 284 \\
\hline & $\mathrm{s} 2 \mathrm{i} 08$ & 341 & 370 & $3.2 \mathrm{D}-16$ & $1.3 \mathrm{D}-08$ & 227 & (1) & 6081.33 & 1118 \\
\hline & s2i09 & 331 & 363 & $4.1 \mathrm{D}-15$ & $6.9 \mathrm{D}-08$ & 733 & $(465)$ & 8453.13 & 1269 \\
\hline & $\mathrm{s} 2 \mathrm{i} 10$ & 323 & 360 & $3.2 \mathrm{D}-16$ & $1.3 \mathrm{D}-08$ & 1188 & $(521)$ & 13996.47 & 899 \\
\hline & s2i11 & 296 & 350 & $2.6 \mathrm{D}-16$ & $1.1 \mathrm{D}-08$ & 1468 & $(1070)$ & 9254.16 & 2355 \\
\hline & s2i12 & 265 & 336 & $1.1 \mathrm{D}-16$ & $1.2 \mathrm{D}-08$ & 5337 & (4128) & 20337.15 & 413 \\
\hline \multirow{6}{*}{ 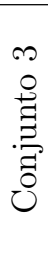 } & s3i01 & 87 & 139 & $6.6 \mathrm{D}-16$ & $1.1 \mathrm{D}-08$ & 56217 & $(53195)$ & 18557.22 & 10011 \\
\hline & s3i02 & 56 & 61 & $2.2 \mathrm{D}-17$ & $2.7 \mathrm{D}-09$ & 57007 & $(56811)$ & 3050.48 & 443059 \\
\hline & s3i03 & 107 & 141 & $6.4 \mathrm{D}-15$ & $1.1 \mathrm{D}-07$ & 17797 & $(14492)$ & 5538.99 & 53725 \\
\hline & s3i04 & 49 & 78 & $9.4 \mathrm{D}-20$ & $4.0 \mathrm{D}-10$ & 636 & (105) & 81.29 & 192575 \\
\hline & s3i05 & 128 & 151 & $3.6 \mathrm{D}-16$ & $7.0 \mathrm{D}-09$ & 3488 & (2797) & 2443.46 & 31326 \\
\hline & s3i06 & 59 & 79 & $4.4 \mathrm{D}-09$ & $5.1 \mathrm{D}-05$ & 8612 & $(8158)$ & 835.34 & 240445 \\
\hline
\end{tabular}

Tabela 4.11: Desempenho do Algoritmo 4.1 associado à estratégia M2 para o cálculo dos pontos iniciais. 


\begin{tabular}{|c|c|c|c|c|c|c|c|c|c|}
\hline \multicolumn{3}{|c|}{ Instância } & \multicolumn{6}{|c|}{ Solução e esforço computacional } & Esforço extra \\
\hline & Nome & $m_{l b}$ & $m$ & $f^{\xi}$ & $\left\|c^{\xi}\right\|_{\infty}$ & & $\mathrm{PI}$ & Tempo de CPU (s.) & \#PIA \\
\hline \multirow{14}{*}{ 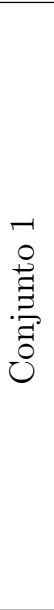 } & sli01 & 4 & 5 & $9.0 \mathrm{D}-19$ & $7.9 \mathrm{D}-10$ & 5 & $(5)$ & 0.00 & 17482175 \\
\hline & s1i02 & 12 & 13 & $4.2 \mathrm{D}-17$ & 7.7D-09 & 5 & $(5)$ & 0.03 & 4059198 \\
\hline & s1i03 & 20 & 24 & $9.6 \mathrm{D}-17$ & $1.1 \mathrm{D}-08$ & 4 & (1) & 0.05 & 727197 \\
\hline & s1i04 & 36 & 39 & $1.5 \mathrm{D}-16$ & 8.9D-09 & 26 & $(21)$ & 2.24 & 195840 \\
\hline & s1i05 & 51 & 57 & $9.5 \mathrm{D}-16$ & $3.2 \mathrm{D}-08$ & 15 & $(10)$ & 3.56 & 77726 \\
\hline & s1i06 & 74 & 78 & $1.3 \mathrm{D}-16$ & 1.1D-08 & 16 & (12) & 20.14 & 29734 \\
\hline & s1i07 & 95 & 103 & $2.2 \mathrm{D}-16$ & $1.0 \mathrm{D}-08$ & 52 & $(45)$ & 65.49 & 20173 \\
\hline & s1i08 & 126 & 132 & 2.9D-17 & 5.7D-09 & 243 & $(225)$ & 713.19 & 7663 \\
\hline & s1i09 & 159 & 164 & $3.1 \mathrm{D}-16$ & $1.2 \mathrm{D}-08$ & 191 & (124) & 961.63 & 5963 \\
\hline & s1i10 & 192 & 199 & $3.7 \mathrm{D}-16$ & $1.8 \mathrm{D}-08$ & 214 & (196) & 1678.21 & 2906 \\
\hline & s1i11 & 238 & 238 & - & - & - & - & - & 2271 \\
\hline & s1i12 & 273 & 281 & $3.3 \mathrm{D}-16$ & $1.1 \mathrm{D}-08$ & 10 & (3) & 226.39 & 1906 \\
\hline & s1i13 & 320 & 327 & $1.8 \mathrm{D}-16$ & $1.0 \mathrm{D}-08$ & 291 & $(267)$ & 7287.23 & 873 \\
\hline & s1i14 & 374 & 376 & $2.7 \mathrm{D}-15$ & 3.3D-08 & 4 & (3) & 356.83 & 944 \\
\hline \multirow{12}{*}{ 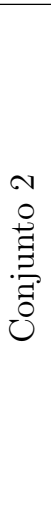 } & s2i01 & 369 & 375 & $1.9 \mathrm{D}-15$ & $5.7 \mathrm{D}-08$ & 6 & (1) & 557.13 & 724 \\
\hline & s2i02 & 367 & 375 & $2.6 \mathrm{D}-16$ & $1.7 \mathrm{D}-08$ & 8 & (1) & 143.56 & 982 \\
\hline & s2i03 & 363 & 375 & $3.7 \mathrm{D}-16$ & $1.8 \mathrm{D}-08$ & 444 & (178) & 16654.77 & 162 \\
\hline & s2i04 & 364 & 377 & $1.8 \mathrm{D}-16$ & $9.5 \mathrm{D}-09$ & 162 & (1) & 7741.19 & 387 \\
\hline & s2i05 & 361 & 369 & 4.0D-17 & 6.8D-09 & 64 & (1) & 2913.40 & 328 \\
\hline & s2i06 & 354 & 373 & 4.6D-17 & 2.8D-09 & 327 & $(126)$ & 12170.68 & 290 \\
\hline & s2i07 & 346 & 371 & $2.5 \mathrm{D}-16$ & $1.2 \mathrm{D}-08$ & 710 & $(261)$ & 18706.98 & 160 \\
\hline & s2i08 & 341 & 366 & $1.0 \mathrm{D}-08$ & $6.0 \mathrm{D}-05$ & 836 & $(1)$ & 17273.26 & 144 \\
\hline & s2i09 & 331 & 358 & $1.9 \mathrm{D}-15$ & $5.6 \mathrm{D}-08$ & 915 & (132) & 20169.86 & 140 \\
\hline & s2i10 & 323 & 357 & 4.1D-16 & 1.1D-08 & 2416 & (106) & 21095.57 & 57 \\
\hline & s2i11 & 296 & 345 & 2.6D-16 & 1.6D-08 & 1266 & (1) & 19803.39 & 321 \\
\hline & s2i12 & 265 & 331 & $5.1 \mathrm{D}-17$ & 3.9D-09 & 2167 & $(1)$ & 16411.58 & 801 \\
\hline \multirow{6}{*}{ 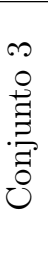 } & s3i01 & 87 & 134 & $5.7 \mathrm{D}-17$ & $5.3 \mathrm{D}-09$ & 23054 & $(15296)$ & 7248.47 & 58554 \\
\hline & s3i02 & 56 & 61 & 8.0D-18 & $1.4 \mathrm{D}-09$ & 348243 & $(348074)$ & 16652.31 & 122688 \\
\hline & s3i03 & 107 & 141 & $8.5 \mathrm{D}-16$ & $3.8 \mathrm{D}-08$ & 7052 & (3) & 2530.15 & 50832 \\
\hline & s3i04 & 49 & 78 & $5.2 \mathrm{D}-17$ & 5.0D-09 & 111922 & (105478) & 11339.10 & 97496 \\
\hline & s3i05 & 128 & 150 & 4.1D-16 & 9.9D-09 & 11719 & (10614) & 8760.11 & 15730 \\
\hline & s3i06 & 59 & 79 & $1.8 \mathrm{D}-16$ & $1.7 \mathrm{D}-08$ & 70963 & $(66584)$ & 7388.61 & 148024 \\
\hline
\end{tabular}

Tabela 4.12: Desempenho do Algoritmo 4.1 associado à estratégia M3 para o cálculo dos pontos iniciais. 


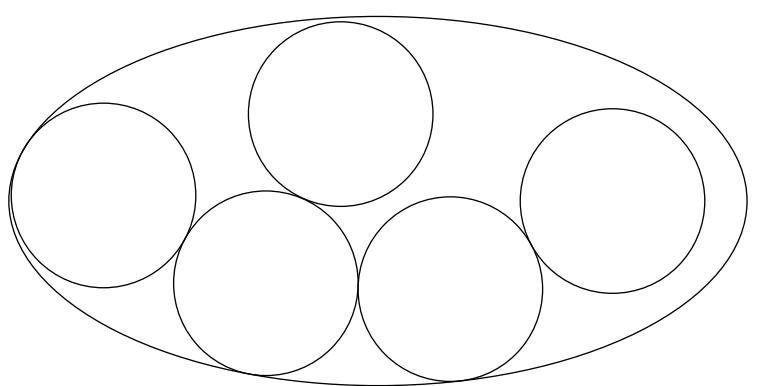

(a) $\mathrm{s} 1 \mathrm{i} 01$

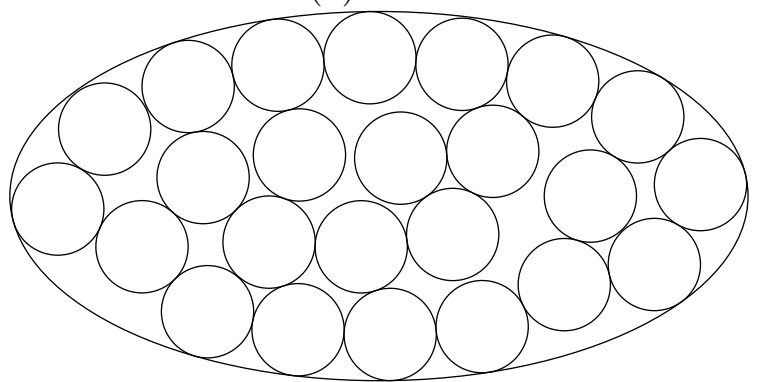

(c) $\mathrm{s} 1 \mathrm{i} 03$

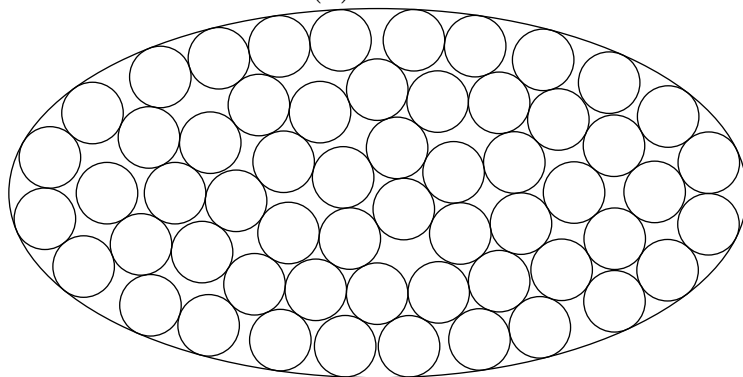

(e) $\mathrm{s} 1 \mathrm{i} 05$

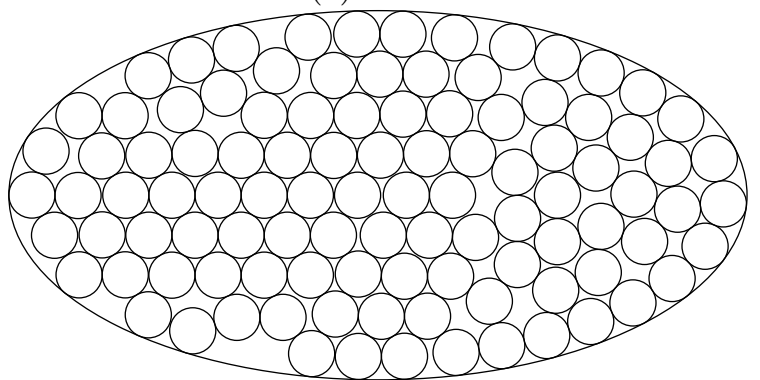

(g) s1i07

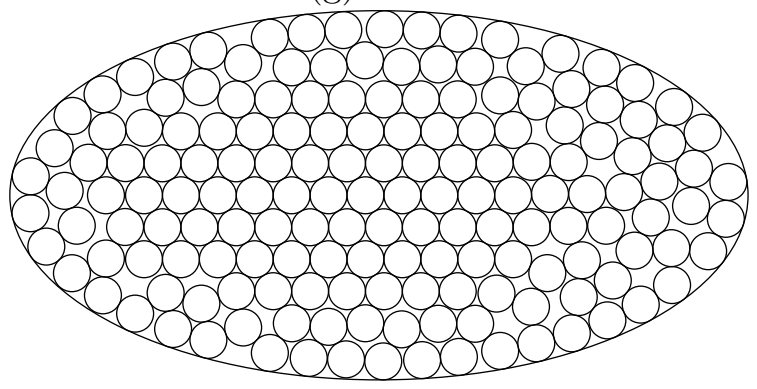

(i) $\mathrm{s} 1 \mathrm{i09}$

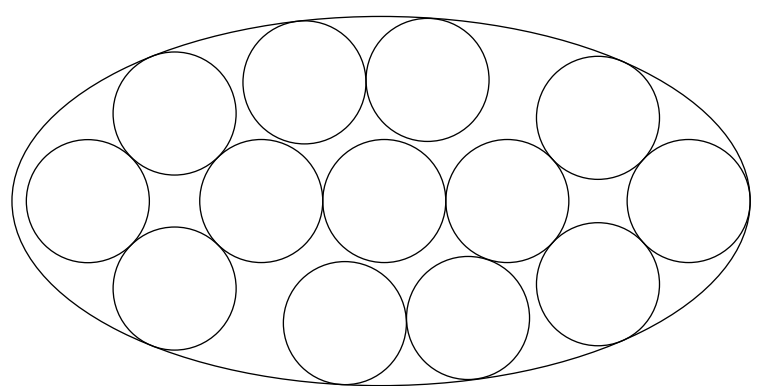

(b) s1i02

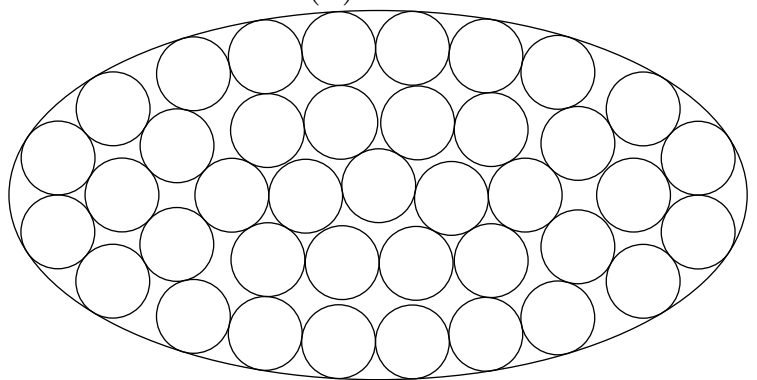

(d) $\mathrm{s} 1 \mathrm{i} 04$

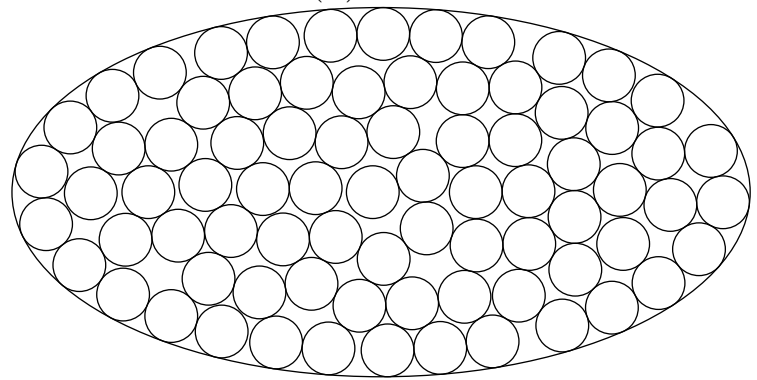

(f) $\mathrm{s} 1 \mathrm{i06}$

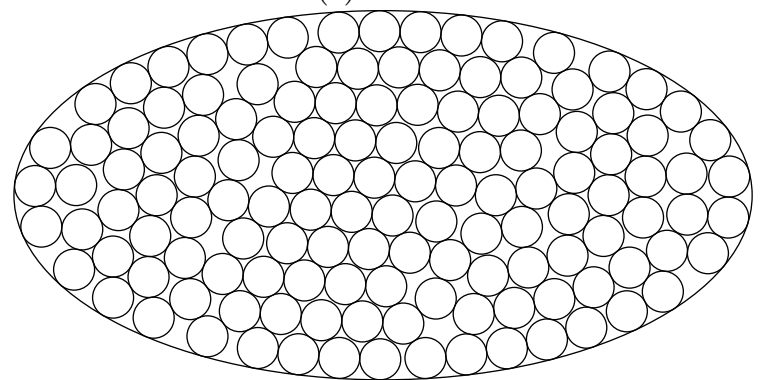

(h) s1i08

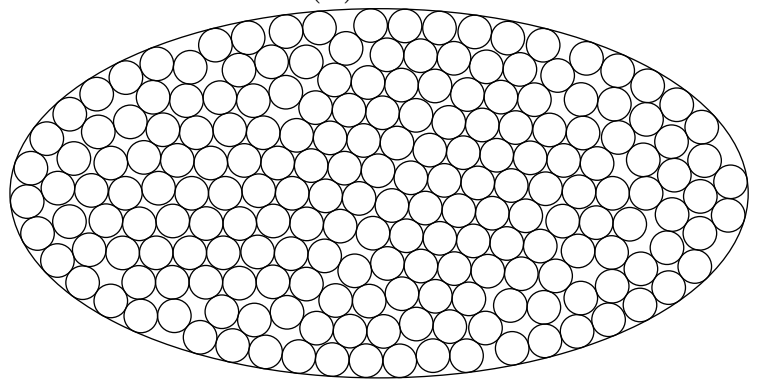

(j) s1i10

Figura 4.12: Representações gráficas das soluções obtidas pelas estratégias M1 e/ou M2 para as instâncias s1i01-s1i10 do conjunto 1. 


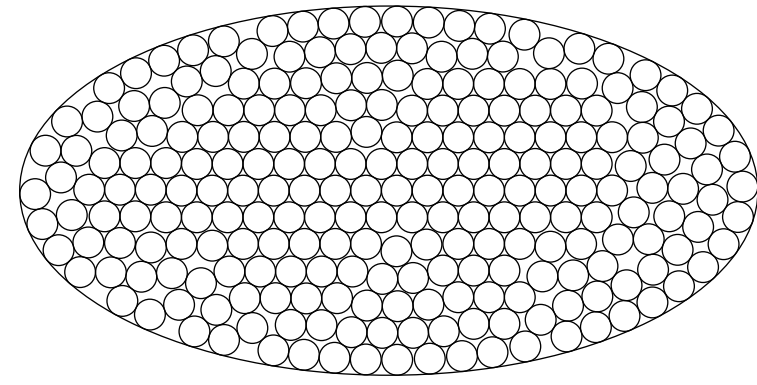

(k) s1i11

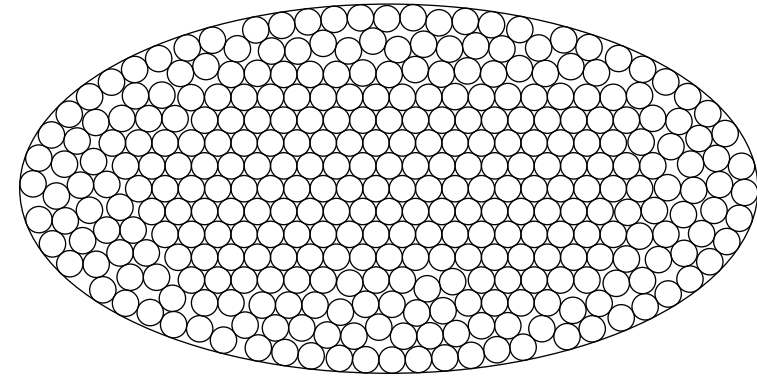

(m) s1i13

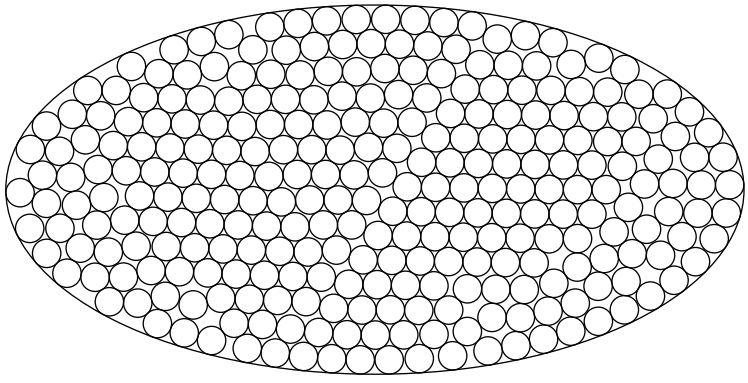

(1) s1i12

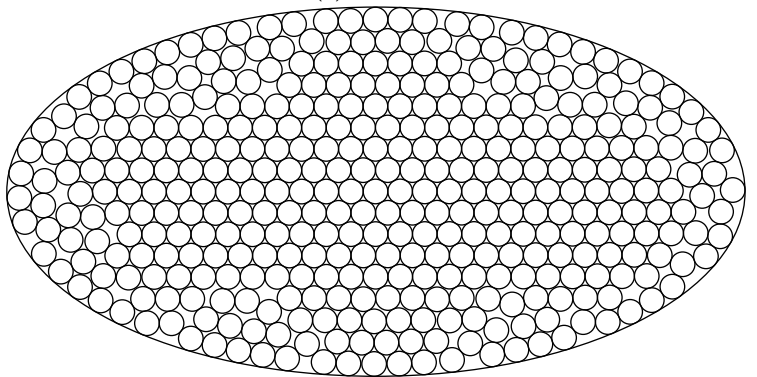

(n) s1i14

Figura 4.13: Representações gráficas das soluções obtidas pelas estratégias M1 e/ou M2 para as instâncias s1i11-s1i14 do conjunto 1. 


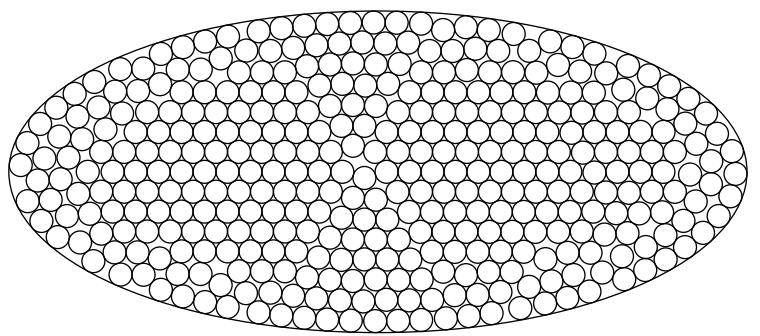

(a) s2i01

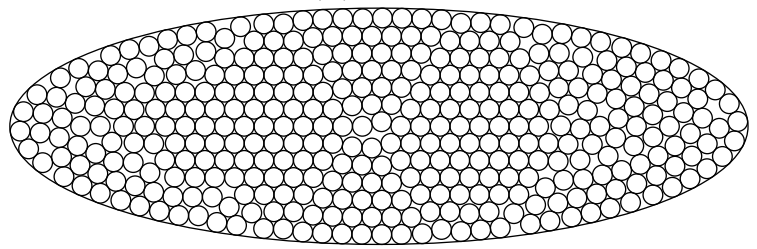

(c) $\mathrm{s} 2 \mathrm{i} 03$

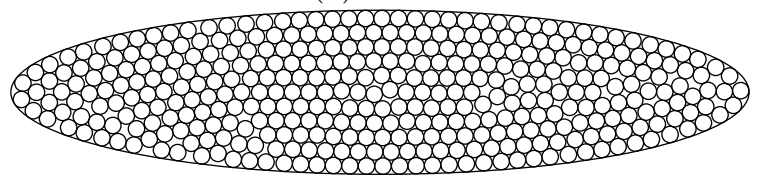

(e) $\mathrm{s} 2 \mathrm{i} 05$

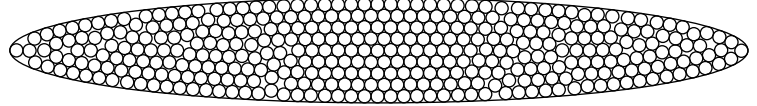

(g) s2i07

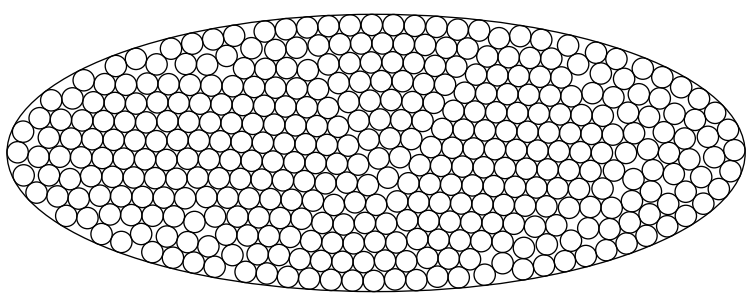

(b) s2i02

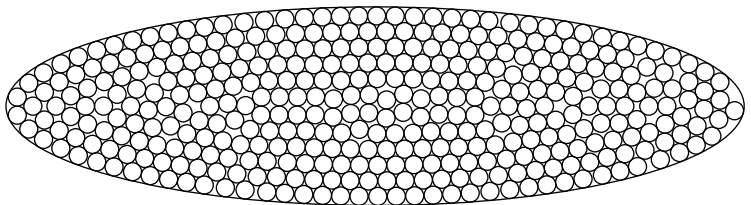

(d) s2i04

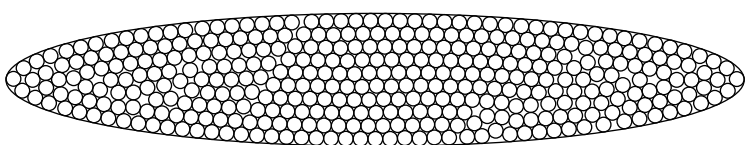

(f) s2i06

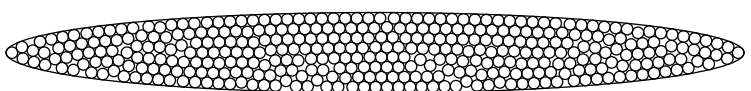

(h) s2i08

Figura 4.14: Representações gráficas das soluções obtidas pelas estratégias M1 e/ou M2 para as instâncias s2i01-s2i08 do conjunto 2.

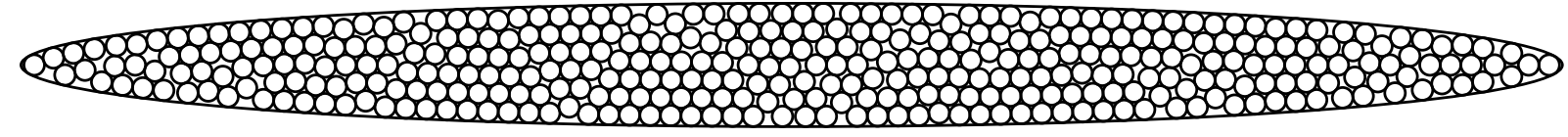

(i) $\mathrm{s} 2 \mathrm{i} 09$

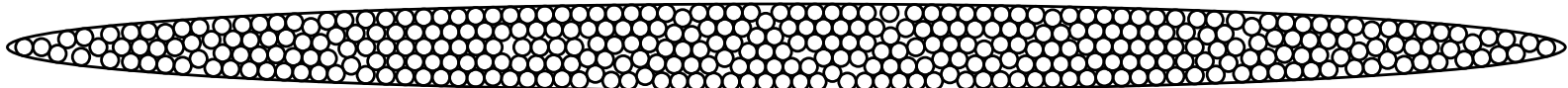

(j) $s 2 \mathrm{i} 10$ cos

(k) s2i11

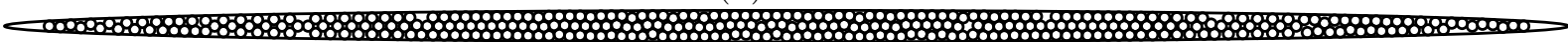

(l) $\mathrm{s} 2 \mathrm{i} 12$

Figura 4.15: Representações gráficas das soluções obtidas pelas estratégias M1 e/ou M2 para as instâncias s2i09-s2i12 do conjunto 2. 


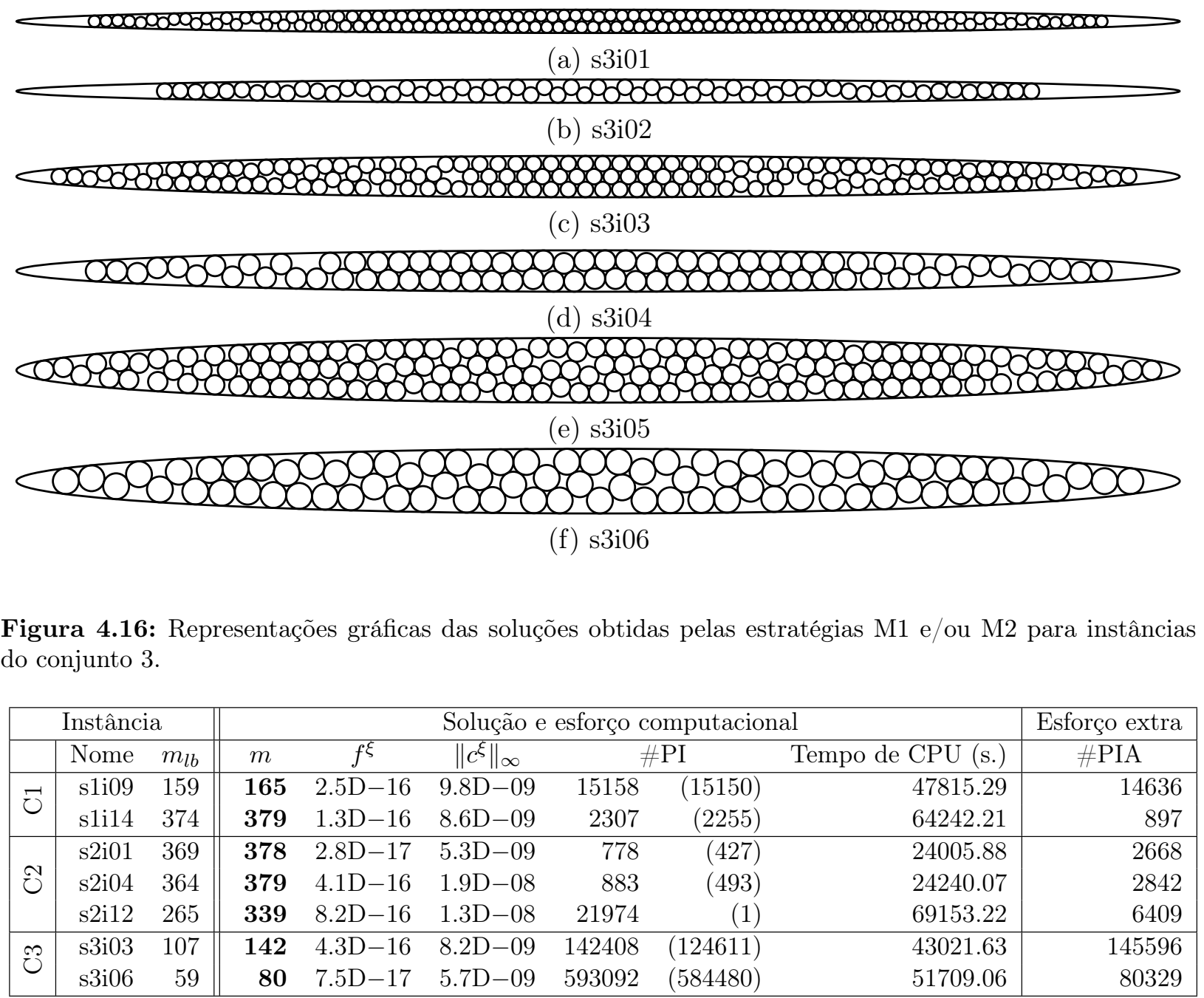

Tabela 4.13: Desempenho do Algoritmo 4.1 associado à estratégia M2 por 24 horas.

Como a estratégia M2 apresentou o melhor desempenho, rodamos a estratégia M2 com um limite de tempo de 24 horas. A Tabela 4.13 mostra 7 instâncias em que esta rodada da estratégia M2 foi capaz de melhorar as melhores soluções encontradas. A Figura 4.17 ilustra estas soluções encontradas.

\subsection{Resumindo os melhores resultados}

No decorrer deste capítulo, descrevemos várias tentativas de encontrar soluções globais para as 32 instâncias nos conjuntos 1-3. Neste momento, é importante ressaltar que as soluções obtidas satisfazem as precisões requeridas em (4.6) e (4.7), descritas no início deste capítulo, e que, claramente, soluções "melhores" poderiam ser encontradas se relaxássemos esses requerimentos como também algumas das soluções apresentadas poderiam ser consideradas soluções se tolerâncias mais rigorosas fossem consideradas. Independentemente das exigências de tolerâncias de otimização (4.6) e (4.7), também reportamos, para cada solução dada, os valores reais de $f^{\xi}$ e $\left\|c^{\xi}\right\|_{\infty}$ em (4.8) e (4.9), respectivamente. Entretanto, esses valores combinam dois tipos de quantidades que devem ser reportadas separadamente. Estas duas medidas são:

Máxima violação absoluta de sobreposição : fortemente relacionado a $\kappa_{i j}^{\xi}$ em (4.2) e dado 


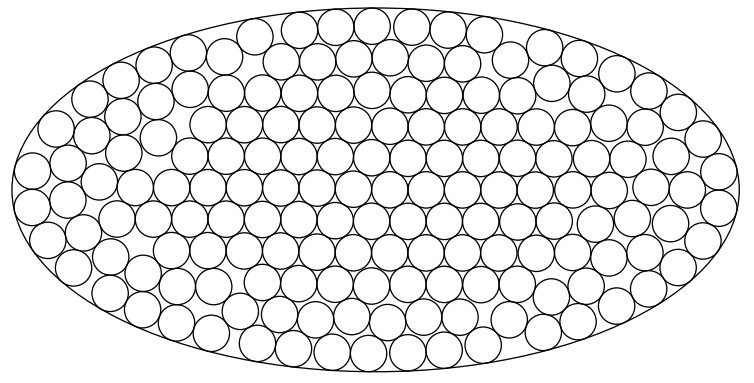

(a) s1i09

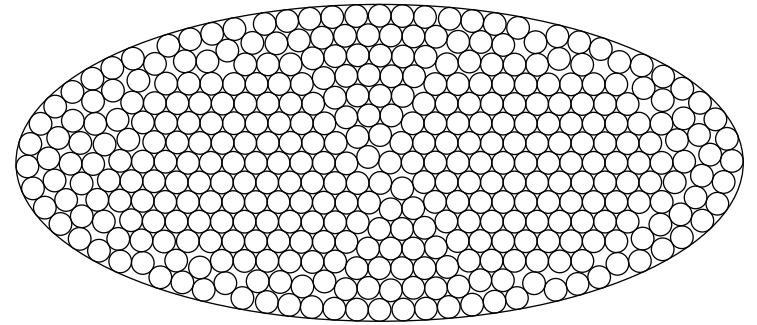

(c) $\mathrm{s} 2 \mathrm{i} 01$

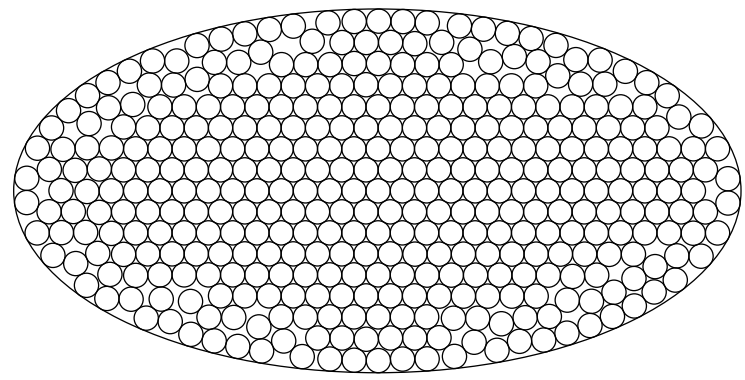

(b) s1i14

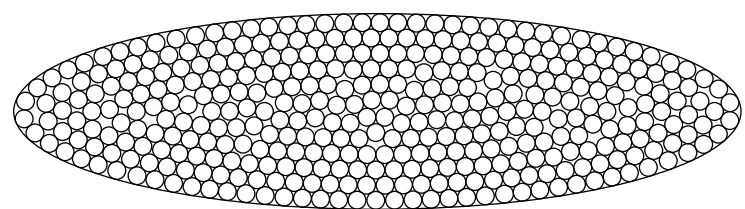

(d) s2i04

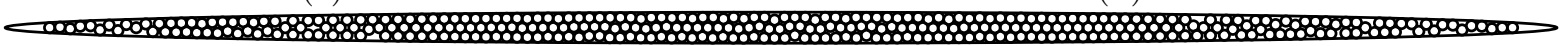

(e) $\mathrm{s} 2 \mathrm{i} 12$

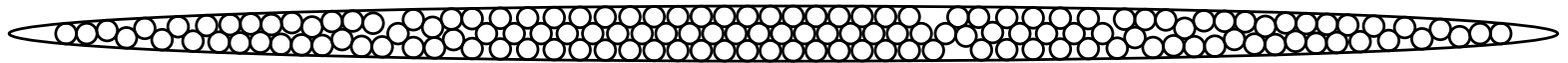

(f) $\mathrm{s} 3 \mathrm{i} 03$

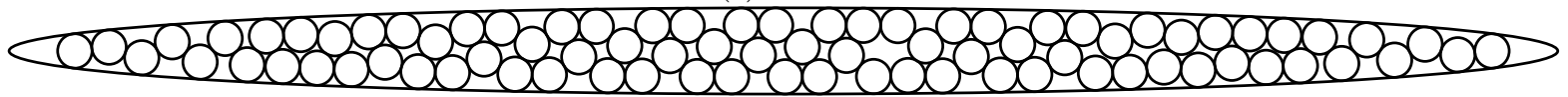

(g) s3i06

Figura 4.17: Representação gráfica das soluções obtidas pela estratégia M2 usando um limite de tempo de CPU de 24 horas e que melhoraram as melhores soluções encontradas pelas estratégias M1-M3 usando um limite de tempo de 6 horas. 
por

$$
\max _{i<j}\left\{\left[2 r-\sqrt{\left\{\left(1+\left(s_{i}-1\right)\left(b^{2} / a^{2}\right)\right) u_{i}-\left(1+\left(s_{j}-1\right)\left(b^{2} / a^{2}\right)\right) u_{j}\right\}^{2}+\left\{s_{i} v_{i}-s_{j} v_{j}\right\}^{2}}\right]_{+}\right\},
$$

Máxima violação absoluta do contêiner : fortemente relacionado a $g_{i}^{\xi}$ em (4.2) e dado por

$$
\max _{i}\left\{\left[r-\left(s_{i}-1\right) \sqrt{\left(b^{2} / a^{2}\right)^{2} u_{i}^{2}+v_{i}^{2}}\right]_{+}\right\}
$$

A Tabela 4.14 resume esses resultados apontando para cada solução presente no texto. Desenvolver uma estratégia mais eficiente capaz de encontrar por si só todas as melhores soluções reportadas aqui (com as tolerâncias estabelecidas) permanece um problema em aberto. 


\begin{tabular}{|c|c|c|c|c|c|c|c|c|c|}
\hline \multicolumn{2}{|c|}{ Instância } & \multicolumn{8}{|c|}{ Solução } \\
\hline & Nome & $m$ & $f^{\xi}$ & $\left\|c^{\xi}\right\|_{\infty}$ & MVAS & MVAC & densidade & Estratégia & Gráfico \\
\hline \multirow{14}{*}{ 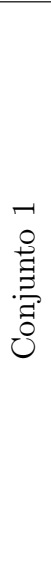 } & s1i01 & 5 & $4.1 \mathrm{D}-19$ & $7.8 \mathrm{D}-10$ & $0.0 \mathrm{D}+00$ & $0.0 \mathrm{D}+00$ & 0.6250 & $\mathrm{R} 4 / \mathrm{T} / \mathrm{M} 1 / \mathrm{M} 2 / \mathrm{M} 3$ & Fig. 4.12(a) \\
\hline & s1i02 & 13 & $1.5 \mathrm{D}-16$ & $1.5 \mathrm{D}-08$ & $4.8 \mathrm{D}-10$ & $8.4 \mathrm{D}-11$ & 0.7222 & $\mathrm{R} 4 / \mathrm{T} / \mathrm{M} 1 / \mathrm{M} 2 / \mathrm{M} 3$ & Fig. 4.12(b) \\
\hline & s1i03 & 24 & $9.6 \mathrm{D}-17$ & $1.1 \mathrm{D}-08$ & $9.4 \mathrm{D}-10$ & $1.1 \mathrm{D}-10$ & 0.7500 & $\mathrm{R} 4 / \mathrm{T} / \mathrm{M} 1 / \mathrm{M} 2 / \mathrm{M} 3$ & Fig. $4.12(\mathrm{c})$ \\
\hline & s1i04 & 39 & $2.7 \mathrm{D}-17$ & $3.8 \mathrm{D}-09$ & $2.8 \mathrm{D}-09$ & $2.1 \mathrm{D}-10$ & 0.7800 & $\mathrm{R} 4 / \mathrm{T} / \mathrm{M} 1 / \mathrm{M} 2 / \mathrm{M} 3$ & Fig. $4.12(\mathrm{~d})$ \\
\hline & $\mathrm{s} 1 \mathrm{i} 05$ & 57 & $4.5 \mathrm{D}-17$ & $9.1 \mathrm{D}-09$ & $9.1 \mathrm{D}-09$ & $8.8 \mathrm{D}-11$ & 0.7917 & T/M1/M2/M3 & Fig. 4.12(e) \\
\hline & s1i06 & 78 & $7.2 \mathrm{D}-17$ & $5.6 \mathrm{D}-09$ & $1.4 \mathrm{D}-09$ & $2.6 \mathrm{D}-10$ & 0.7959 & $\mathrm{~T} / \mathrm{M} 1 / \mathrm{M} 2 / \mathrm{M} 3$ & Fig. 4.12(f) \\
\hline & $\mathrm{s} 1 \mathrm{i07}$ & 103 & $9.5 \mathrm{D}-17$ & $7.6 \mathrm{D}-09$ & $2.3 \mathrm{D}-09$ & $3.8 \mathrm{D}-10$ & 0.8047 & $\mathrm{~T} / \mathrm{M} 1 / \mathrm{M} 2 / \mathrm{M} 3$ & Fig. 4.12(g) \\
\hline & $\mathrm{s} 1 \mathrm{i} 08$ & 132 & $1.0 \mathrm{D}-15$ & $2.1 \mathrm{D}-08$ & $2.7 \mathrm{D}-09$ & $6.4 \mathrm{D}-10$ & 0.8148 & $\mathrm{~T} / \mathrm{M} 1 / \mathrm{M} 2 / \mathrm{M} 3$ & Fig. 4.12(h) \\
\hline & s1i09 & 165 & $2.5 \mathrm{D}-16$ & $9.8 \mathrm{D}-09$ & $1.6 \mathrm{D}-09$ & $3.5 \mathrm{D}-10$ & 0.8250 & $\mathrm{M} 2^{+}$ & Fig. 4.17(a) \\
\hline & s1i10 & 199 & $1.8 \mathrm{D}-16$ & $1.5 \mathrm{D}-08$ & $1.6 \mathrm{D}-09$ & $3.2 \mathrm{D}-10$ & 0.8223 & $\mathrm{~T} / \mathrm{M} 2 / \mathrm{M} 3$ & Fig. 4.12(j) \\
\hline & s1i11 & 239 & $3.6 \mathrm{D}-16$ & $2.3 \mathrm{D}-08$ & $2.1 \mathrm{D}-09$ & $5.7 \mathrm{D}-10$ & 0.8299 & $\mathrm{~T} / \mathrm{M} 1 / \mathrm{M} 2$ & Fig. 4.13(k) \\
\hline & s1i12 & 281 & $2.2 \mathrm{D}-17$ & $2.5 \mathrm{D}-09$ & $6.7 \mathrm{D}-10$ & $1.5 \mathrm{D}-10$ & 0.8314 & M1/M3 & Fig. 4.13(1) \\
\hline & s1i13 & 328 & $1.1 \mathrm{D}-09$ & $2.1 \mathrm{D}-05$ & $2.3 \mathrm{D}-06$ & $7.0 \mathrm{D}-07$ & 0.8367 & $\mathrm{~T} / \mathrm{M} 2$ & Fig. $4.13(\mathrm{~m})$ \\
\hline & s1i14 & 379 & $1.3 \mathrm{D}-16$ & $8.6 \mathrm{D}-09$ & $1.0 \mathrm{D}-09$ & $2.1 \mathrm{D}-10$ & 0.8422 & $\mathrm{M} 2^{+}$ & Fig. 4.17(b) \\
\hline \multirow{12}{*}{ 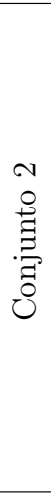 } & $\mathrm{s} 2 \mathrm{i} 01$ & 378 & $2.8 \mathrm{D}-17$ & $5.3 \mathrm{D}-09$ & $1.4 \mathrm{D}-09$ & $9.0 \mathrm{D}-11$ & 0.8400 & $\mathrm{M}^{+}$ & Fig. 4.17(c) \\
\hline & s2i02 & 377 & $6.1 \mathrm{D}-17$ & $5.5 \mathrm{D}-09$ & $8.9 \mathrm{D}-10$ & $1.2 \mathrm{D}-10$ & 0.8378 & M1 & Fig. 4.14(b) \\
\hline & $\mathrm{s} 2 \mathrm{i} 03$ & 376 & $7.6 \mathrm{D}-16$ & $1.9 \mathrm{D}-08$ & $1.3 \mathrm{D}-09$ & $4.1 \mathrm{D}-10$ & 0.8356 & $\mathrm{M} 1 / \mathrm{M} 2$ & Fig. 4.14(c) \\
\hline & $\mathrm{s} 2 \mathrm{i} 04$ & 379 & $4.1 \mathrm{D}-16$ & $1.9 \mathrm{D}-08$ & $1.3 \mathrm{D}-09$ & $2.5 \mathrm{D}-10$ & 0.8422 & $\mathrm{M}^{+}$ & Fig. 4.17(d) \\
\hline & $\mathrm{s} 2 \mathrm{i} 05$ & 379 & $4.1 \mathrm{D}-16$ & $1.4 \mathrm{D}-08$ & $1.5 \mathrm{D}-09$ & $3.6 \mathrm{D}-10$ & 0.8422 & M2 & Fig. 4.14(e) \\
\hline & $\mathrm{s} 2 \mathrm{i} 06$ & 378 & $3.8 \mathrm{D}-16$ & $1.2 \mathrm{D}-08$ & $1.4 \mathrm{D}-09$ & $3.1 \mathrm{D}-10$ & 0.8400 & M2 & Fig. 4.14(f) \\
\hline & $\mathrm{s} 2 \mathrm{i} 07$ & 375 & $1.4 \mathrm{D}-09$ & $2.0 \mathrm{D}-05$ & $3.1 \mathrm{D}-06$ & $7.6 \mathrm{D}-07$ & 0.8333 & M2 & Fig. 4.14(g) \\
\hline & $\mathrm{s} 2 \mathrm{i} 08$ & 370 & $3.2 \mathrm{D}-16$ & $1.3 \mathrm{D}-08$ & $2.5 \mathrm{D}-09$ & $5.7 \mathrm{D}-10$ & 0.8222 & M2 & Fig. 4.14(h) \\
\hline & s2i09 & 365 & $7.7 \mathrm{D}-17$ & $3.5 \mathrm{D}-09$ & $1.7 \mathrm{D}-09$ & $2.4 \mathrm{D}-10$ & 0.8111 & M1 & Fig. 4.15(i) \\
\hline & $\mathrm{s} 2 \mathrm{i} 10$ & 360 & $3.2 \mathrm{D}-16$ & $1.3 \mathrm{D}-08$ & $2.9 \mathrm{D}-09$ & $1.2 \mathrm{D}-09$ & 0.8000 & M2 & Fig. $4.15(\mathrm{j})$ \\
\hline & $\mathrm{s} 2 \mathrm{i} 11$ & 353 & $5.1 \mathrm{D}-16$ & $2.0 \mathrm{D}-08$ & $4.7 \mathrm{D}-09$ & $1.4 \mathrm{D}-09$ & 0.7844 & M1 & Fig. $4.15(\mathrm{k})$ \\
\hline & s2i12 & 339 & $8.2 \mathrm{D}-16$ & $1.3 \mathrm{D}-08$ & $5.8 \mathrm{D}-09$ & $2.2 \mathrm{D}-09$ & 0.7533 & $\mathrm{M}^{+}$ & Fig. 4.17(e) \\
\hline \multirow{6}{*}{ 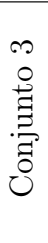 } & s3i01 & 139 & $6.6 \mathrm{D}-16$ & $1.1 \mathrm{D}-08$ & 7.9D-09 & $2.7 \mathrm{D}-09$ & 0.6950 & M2 & Fig. 4.16(a) \\
\hline & $\mathrm{s} 3 \mathrm{i} 02$ & 61 & $2.2 \mathrm{D}-17$ & $2.7 \mathrm{D}-09$ & $2.7 \mathrm{D}-09$ & $6.6 \mathrm{D}-10$ & 0.5422 & M2/M3 & Fig. 4.16(b) \\
\hline & s3i03 & 142 & $4.3 \mathrm{D}-16$ & $8.2 \mathrm{D}-09$ & $4.4 \mathrm{D}-09$ & $1.4 \mathrm{D}-09$ & 0.7100 & $\mathrm{M} 2^{+}$ & Fig. 4.17(f) \\
\hline & s3i04 & 78 & $9.4 \mathrm{D}-20$ & $4.0 \mathrm{D}-10$ & $0.0 \mathrm{D}+00$ & $6.0 \mathrm{D}-12$ & 0.6933 & M2/M3 & Fig. 4.16(d) \\
\hline & s3i05 & 151 & $3.6 \mathrm{D}-16$ & $7.0 \mathrm{D}-09$ & $3.6 \mathrm{D}-09$ & $1.0 \mathrm{D}-09$ & 0.7550 & M2 & Fig. 4.16(e) \\
\hline & s3i06 & 80 & $7.5 \mathrm{D}-17$ & $5.7 \mathrm{D}-09$ & $5.7 \mathrm{D}-09$ & $9.8 \mathrm{D}-10$ & 0.7111 & $\mathrm{M}^{+}{ }^{+}$ & Fig. 4.17(g) \\
\hline
\end{tabular}

Tabela 4.14: Resumo das melhores soluções encontradas. ${ }^{1}$

\footnotetext{
${ }^{1} \mathrm{O}$ "T" na coluna "estratégia" representa que a solução também foi encontrada pelo Algoritmo 4.2 utilizando alguns dos pontos iniciais dos tipos R1, R2, R3 ou R4.
} 


\section{Capítulo 5}

\section{Conclusões}

Neste trabalho, diversas estratégias de otimização global foram aplicadas ao problema de empacotamento de círculos em elipses. O modelo introduzido em [Cal12] pode então ser testado em diversas instâncias do problema de determinar o número máximo de círculos que podem ser empacotados em um contêiner elíptico.

Desenvolvemos diversas estratégias para gerar pontos iniciais aleatórios e para modificar pontos estacionários. Quando é requerido que a modificação do ponto estacionário forneça um ponto com um valor de função objetivo menor que o valor da função objetivo no ponto estacionário, caraterizase uma estratégia de tunneling. Surpreendentemente, os experimentos computacionais mostraram que os casos em que isso não ocorre também geram pontos iniciais de "boa qualidade" que, eventualmente, levam a uma solução global. De forma geral, as estratégias de tunneling apresentaram resultados superiores as estratégias rudimentares de multistart, mas foram superadas por estratégias de multistart que constroem os pontos iniciais usando como base pontos estacionários de rodadas anteriores.

A estratégia básica para empacotar o maior número possível de círculos numa elipse dada (utilizada em diversas publicações [BMR05, BL10, BMMR06, BMNR06]) consiste em resolver uma sequência de problemas de viabilidade com um número crescente (de um em um) de círculos. O argumento para utilizar esta estratégia sequencial no lugar de alguma outra estratégia baseada em bisseção é que problemas com um número próximo do ótimo ou ainda com mais círculos do que de fato podem ser empacotados são difíceis e computacionalmente custosos, enquanto que problemas "folgados" com um número pequeno de círculos são fáceis de resolver. Mesmo diante disto, a estratégia geral pode ser melhorada se conhecermos um limitante inferior no número de itens que podem ser empacotados e uma solução associada a este limitante. Para tanto, desenvolvemos uma estratégia para o cálculo de limitantes inferiores baseada em reticulados hexagonais. Os experimentos computacionais mostraram que soluções iniciais baseadas em reticulados hexagonais fornecem aproximações de boa qualidade em instâncias com um número muito grande de círculos, sugerindo que procedimentos de otimização possam não ser a ferramenta mais adequada nestes casos.

De forma geral, desenvolvemos estratégias de otimização e apresentamos os primeiros resultados computacionais utilizando o modelo introduzido em [Cal12] para empacotar círculos em elipses. Este é um problema com aplicações interessantes em biologia (veja, por exemplo, [UW12]) e que recentemente tem despertado o interesse de outros pesquisadores da área. Pretendemos no futuro analisar a possibilidade de adaptar os modelos e as técnicas aqui apresentadas para o problema de empacotar elipses em elipses. 
CONCLUSÕES 


\section{Referências Bibliográficas}

[ABMS07] R. Andreani, E. G. Birgin, J. M. Martínez e M. L. Schuverdt. On augmented lagrangian methods with general lower-level constraints. SIAM Journal on Optimization, 18:12861309, 2007. 1, 9, 18

[ABMS08] R. Andreani, E. G. Birgin, J. M. Martínez e M. L. Schuverdt. Augmented lagrangian methods under the constant positive linear dependence constraint qualification. Mathematical Programming, 111:5-32, 2008. 9, 18

[BFM10] E. G. Birgin, C. A. Floudas e J. M. Martínez. Global minimization using an augmented lagrangian method with variable lower-level constraints. Mathematical Programming, 125:139-162, 2010. 9

[BG10] E. G. Birgin e J. M. Gentil. New and improved results for packing identical unitary radius circles within triangles, rectangles and strips. Computers $\mathcal{E}$ Operations Research, 37:1318-1327, 2010. 1, 3, 6

[BGR96] C. Barron, S. Gómez e D. Romero. Archimedean polyhedron structure yields a lower energy atomic cluster. Applied Mathematics Letters, 9:75-78, 1996. 25

[BL10] E. G. Birgin e R. D. Lobato. Orthogonal packing of identical rectangles within isotropic convex regions. Computers $\&$ Industrial Engineering, 59:595-602, 2010. 1, 3, 6, 45

[BM02] E. G. Birgin e J. M. Martínez. Large-scale active-set box-constrained optimization method with spectral projected gradients. Computational Optimization and Applications, 23:101-125, 2002. 1

[BMMR06] E. G. Birgin, J. M. Martínez, W. F. Mascarenhas e D. P. Ronconi. Method of sentinels for packing items within arbitrary convex regions. Journal of the Operational Research Society, 57:735-746, 2006. 1, 3, 45

[BMNR06] E. G. Birgin, J. M. Martínez, F. H. Nishihara e D. P. Ronconi. Orthogonal packing of rectangular items within arbitrary convex regions by nonlinear optimization. Computers E Operations Research, 33:3535-3548, 2006. 1, 3, 6, 45

[BMR05] E. G. Birgin, J. M. Martínez e D. P. Ronconi. Optimizing the packing of cylinders into a rectangular container: A nonlinear approach. European Journal of Operational Research, 160:19-33, 2005. 1, 3, 6, 45

[BS08] E. G. Birgin e F. N. C. Sobral. Minimizing the object dimensions in circle and sphere packing problems. Computers \& Operations Research, 35:2357-2375, 2008. 1, 3, 10, 12, 19

[Cal12] H. F. Callisaya. Empacotamento em quadráticas. 2012. Tese, Doutorado em Matemática Aplicada - Universidade Estadual de Campinas. 4, 7, 45

[Can11] D. W. Cantrell. Mensagem pessoal, 2011. 4 
[CS88] J. H. Conway e N. J. A. Sloane. Sphere Packings, Lattices and Groups. Springer-Verlag, New York, Berlin, Heidelberg, London, Paris, Tokyo, 1988. 1

[Fri11] E. Friedman. Erich's packing center, 2011. 1, 4

[HE81] R. W. Hockney e J. W. Eastwood. Computer Simulation Using Particles. McGraw Hill, New York, 1981. 10

[Hog10] C. M. Hogan. Abiotic factor. Em E. Monosson e C. J. Cleveland, editors, Encyclopedia of Earth. Environmental Information Coalition, 2010. Washington, DC., National Council for Science and the Environment. 1

[LM85] A. V. Levy e A. Montalvo. The tunneling algorithm for the global minimization of functions. SIAM Journal on Scientific and Statistical Computing, 6:15-29, 1985. 25

[MABM09] L. Martínez, R. Andrade, E. G. Birgin e J. M. Martínez. Packmol: A package for building initial configurations for molecular dynamics simulations. Journal of Computational Chemistry, 30:2157-2164, 2009. 1, 10, 12

[MB10] W. F. Mascarenhas e E. G. Birgin. Using sentinels to detect intersections of convex and nonconvex polygons. Computational \& Applied Mathematics, 29:247-267, 2010. 3

[MM03] J. M. Martínez e L. Martínez. Packing optimization for automated generation of complex system's initial configurations for molecular dynamics and docking. Journal of Computational Chemistry, 24:819-825, 2003. 1, 10, 12

[NG09] D. V. Nichita e S. Gómez. Efficient location of multiple global minima for the phase stability problem. Chemical Engineering Journal, 152:251-263, 2009. 25

[Sri06] M. Srinivas. Implementation and evaluation of random tunneling algorithm for chemical engineering applications. Computers and Chemical Engineering, 9:1400-1414, 2006. 25

[UW12] C. Uhler e S. Wright. Packing ellipsoids with overlap. Optimization Online Digest, 2012. 45

[Wei11] E. W. Weisstein. Circle packing. MathWorld-A Wolfram Web Resource, 2011. http://mathworld.wolfram.com/CirclePacking.html. 14 